\title{
Model Based Penalized Smoothing for Panel Data Under a Markov Assumption
}

\author{
by Nikolina Beg
}

A thesis submitted to the Faculty of Graduate and Postdoctoral Affairs in partial fulfillment of the requirements for the degree of

Master of Science in Biostatistics

Carleton University

Ottawa, Ontario

(C)2015, Nikolina Beg 


\begin{abstract}
A flexible model for analyzing multi-state panel count data is presented. Panel data here refers to observing the state of individuals or objects under observation at a discrete set of time points. Panel data are found in many disciplines such as the sciences and medicine. It is assumed that the data are being generated by a continuous time, discrete state space Markov process. The transition intensities are represented with model-based penalty smoothing. This provides a class of models ranging between a fully nonparametric and parametric assumption for the transition intensities. Estimation and inference of the proposed approach is outlined and the finite sample properties of the methodology investigated via simulation.
\end{abstract}




\section{Acknowledgments}

I would like to thank my supervisor, Dr. Jason Nielsen, for his support, guidance and advice throughout this program. This project would not have taken off as it did without his extensive knowledge and patience when teaching. I would like to also thank him and Carleton University for the financial support.

To my parents, Visnja and Darko, thank you for always believing I could do whatever I set my mind to. Agnes, thank you for your constant positive words and patient ears. Finally, thank you to my wonderful fiance Daniel, who encouraged me to always stay motivated and focused. 


\section{Contents}

$\begin{array}{lll}1 & \text { Introduction } & 11\end{array}$

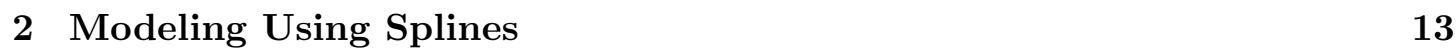

2.1 B-Splines . . . . . . . . . . . . . . . . . . . . . . 13

$2.1 .1 \quad$ Properties of B-Splines . . . . . . . . . . . . . . . . . . . . . 14

2.1 .2 Regression with B-Splines . . . . . . . . . . . . . . . . . . . . . 14

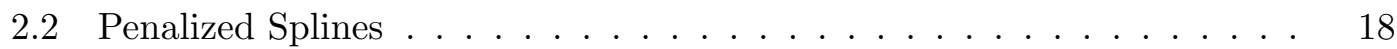

$2.2 .1 \quad$ Derivatives of B-Splines . . . . . . . . . . . . . . . 20

2.2 .2 The Penalty Term . . . . . . . . . . . . . . . . . . . 21

2.2 .3 Model-Based Penalized Splines . . . . . . . . . . . . . . . . 21

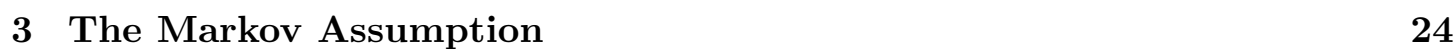

3.1 The Markov Process . . . . . . . . . . . . . . . . . . . . . . . 24

3.1 .1 The Transition Intensity Matrix _... . . . . . . . . . . 25

3.1 .2 The Decomposition Method . . . . . . . . . . . . . . . . 26

\begin{tabular}{|lll}
\hline & Likelihood Analysis and Parameter Estimation & $\mathbf{2 8}$
\end{tabular}

$4.1 \quad$ Likelihood Analysis . . . . . . . . . . . . . . . . . . . . 28

$4.2 \quad$ Fisher's Scoring Method for Parameter Estimation . . . . . . . . . . . . 29

5 Penalized Spline Model for Panel Data Under a Markov Assumption 31

5.1 Penalized Likelihood Analysis . . . . . . . . . . . . . . . 33

5.2 Model-Based Penalties . . . . . . . . . . . . . . . . . . . . . 34

5.2 .1 Derivatives of the Likelihood Function . . . . . . . . . . . . . . 34

5.2 .2 The Score Function . . . . . . . . . . . . . . . . . . . 37

$5.2 .3 \quad$ Fisher's Information Matrix . . . . . . . . . . . . . . . . . . 38

5.2.4 The Weibull Penalty: Maximum Likelihood Parameter Estimates 39

5.3 Estimates of Derived Quantities . . . . . . . . . . . . . . 40

$\begin{array}{lll}6 & \text { Simulation Study } & \mathbf{4 2}\end{array}$

6.1 Methodology . . . . . . . . . . . . . . . . . . . . . . . 43

\begin{tabular}{lll}
\hline 7 & Discussion & 46
\end{tabular}

7.1 Fitting a Weibull Function . . . . . . . . . . . . . . . . . . 47

7.1.1 The Influence of $\lambda$ on Derived Quantities . . . . . . . . . . 53

7.1.2 Coverage Probabilities for the Weibull Function . . . . . . . . . 55

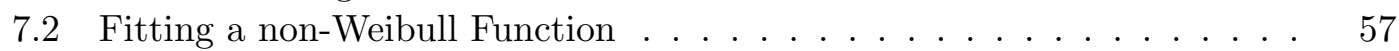

$7.3 \quad$ Using a Second Derivative Penalty for the non-Weibull function . . . . . . 63

7.4 Using a Log-Normal Function with the Model-Based Penalty . . . . . . 65

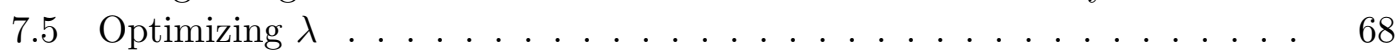

7.5.1 Generalized Cross Validation . . . . . . . . . . . . . . . . 68

7.5 .2 Restricted Maximum Likelihood . . . . . . . . . . . . . . . . . . 69

7.6 Extension to Covariates . . . . . . . . . . . . . . . . . . . . 70

$\begin{array}{lll}8 & \text { Conclusion } & 72\end{array}$ 
\begin{tabular}{|lr}
\hline A Results Appendix & $\mathbf{7 6}$
\end{tabular}

A.1 Case 1: $n=500$ and number of time panels $=50 \ldots \ldots \ldots$. . . . . 76

A.2 Case 2: $n=500$ and number of time panels $=25 \ldots \ldots$. . . . . . . 80

A.3 Case 3: $n=500$ and number of time panels $=10 \ldots \ldots$. . . . . . . 84

A.4 Case 4: $n=250$ and number of time panels $=50 \ldots \ldots 8$

A.5 Case 5: $n=250$ and number of time panels $=25 \ldots \ldots$. . . . . . . . 92

A.6 Case 6: $n=250$ and number of time panels $=10 \ldots \ldots$. . . . . . . 96

A.7 Case 7: $n=150$ and number of time panels $=50 \ldots \ldots \ldots$

A.8 Case 8: $n=150$ and number of time panels $=25$. . . . . . . . . . . . 104

A.9 Case 9: $n=150$ and number of time panels $=10 \ldots \ldots$. . . . . . 108

A.10 Case 10: Quadratic Function with a Divided Difference Penalty . . . . . 108

A.11 Case 11: Fitting a Log-Normal with the Model Based Penalty. . . . . . . 113 
List of Tables

1 Simulation Case Breakdown . . . . . . . . . . . . . . . . . . . 44 


\section{List of Figures}

$1 \quad$ A visual representation of 1(a) and 2(b) degree B-Splines basis functions, as well as their overlapping patterns $[4] . \ldots \ldots \ldots \ldots \ldots \ldots$

$2 \quad$ A B-Spline resulting from coefficients multiplying the cubic basis functions spanning the linear space to which the function is assumed to belong. 17

3 Two models for melanoma data. The CUR model includes the standard second derivative penalty, while the PER model uses a periodic

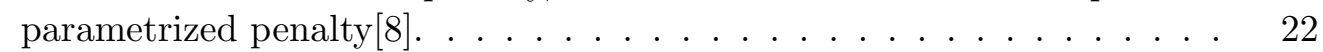

$4 \quad$ Weibull fit in the case with 150 individuals and 10 time panels . . . . . 47

$4 \quad$ Weibull fit in the case with 500 individuals and 50 time panels $\ldots 4$. . . 48

$6 \quad$ Weibull bias in the case with 150 individuals and 10 time panels . . . . 49

\begin{tabular}{llll}
\hline 7 & Weibull bias in the case with 500 individuals and 50 time panels
\end{tabular} . . . . 49

$8 \quad$ Weibull bias in the case with 250 individuals and 10 time panels . . . . 50

$9 \quad$ Weibull bias in the case with 500 individuals and 10 time panels $\ldots . .51$

$10 \quad$ Weibull bias in the case with 500 individuals and 10 time panels. This graph is kept at the same scale as the graphs with fewer individuals. . . 51

$11 \quad$ Weibull bias in the case with 250 individuals and 25 time panels . . . . 52

$12 \quad$ Weibull bias in the case with 250 individuals and 50 time panels . . . . 53

13 Coverage probabilities for $\tilde{q}$ in the case with 150 individuals and 10 time

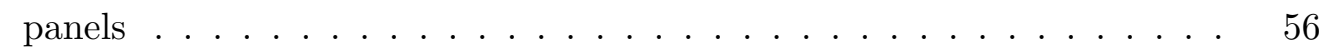

14 Coverage probabilities for $\tilde{q}$ in the case with 500 individuals and 50 time

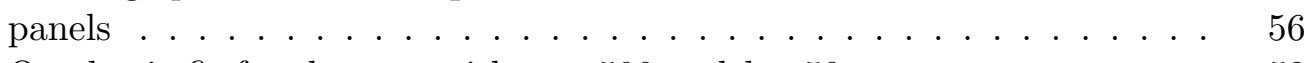

$15 \quad$ Quadratic fit for the case with $n=500$ and $l=50 \ldots \ldots$. . . . . . 58

$16 \quad$ Quadratic fit for the case with $n=250$ and $l=25$. . . . . . . . . 59

$17 \quad$ Quadratic fit for the case with $n=150$ and $l=10 \ldots$. . . . . . . . 60

$18 \quad$ Bias for the case with $n=500$ and $l=50 \ldots \ldots$. . . . . . . . . 61

19 Bias for the case with $n=250$ and $l=10 \ldots \ldots$. . . . . . . 61

$20 \quad$ Coverage probabilities for the case with $n=500$ and $l=50$. . . . . . 62

$21 \quad$ Quadratic fit using the divided difference penalty . . . . . . . . . . . . . 64

$22 \quad$ Quadratic bias using the divided difference penalty . . . . . . . . . . . . 64

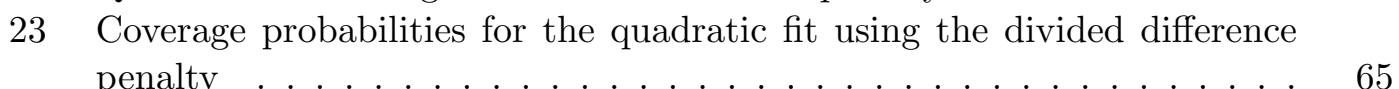

$24 \quad$ Log-normal function fit using the Weibull penalty . . . . . . . . . . . . . . . 66

$25 \quad$ Log-normal function bias using the Weibull penalty. . . . . . . . . . . . 67

$\begin{array}{lll}26 & \text { Log-normal function coverage probabilities using the Weibull penalty . . } 68\end{array}$

A.1 Quadratic fit for Case 1 with $\lambda=11 \ldots \ldots$. . . . . . . . . 76

A.2 $\quad$ Weibull fit for Case 1 with $\lambda=10000 \ldots \ldots$. . . . . . . . . . 77

A.3 $\quad$ Bias for the quadratic function of Case 1 . . . . . . . . . . . . 77

A.4 Bias for the Weibull function of Case 1. We note that the graph is zoomed

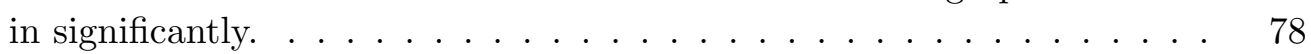

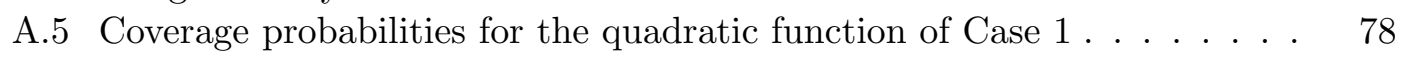

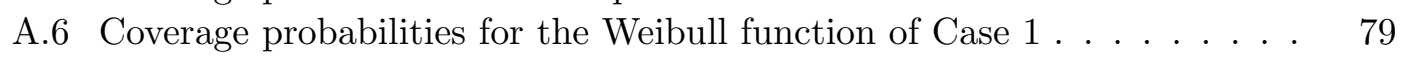

A.7 $\quad$ Quadratic fit for Case 2 with $\lambda=11 \ldots \ldots$. . . . . . . . . . 80

A.8 Weibull fit for Case 2 with $\lambda=10000 \ldots \ldots$. . . . . . . . . . . . 81

A.9 Bias for the quadratic function of Case 2 . . . . . . . . . . . . 81 
A.10 Bias for the Weibull function of Case 2. We note that the graph is zoomed

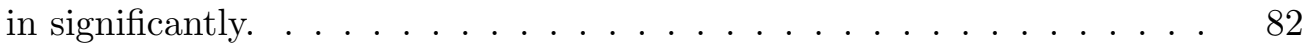

A.11 Coverage probabilities for the quadratic function of Case 2. . . . . . . . 82

A.12 Coverage probabilities for the Weibull function of Case 2 . . . . . . . . 83

A.13 Quadratic fit for Case 3 with $\lambda=27 \ldots \ldots$. . . . . . . . . 84

A.14 Weibull fit for Case 3 with $\lambda=10000$. . . . . . . . . . . . . . . . . 85

A.15 Bias for the quadratic function of Case 3 . . . . . . . . . . . . 85

A.16 Bias for the Weibull function of Case 3. We note that the graph is zoomed

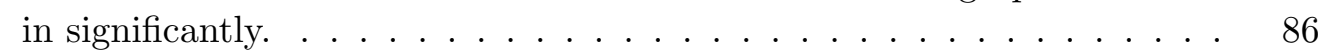

A.17 Coverage probabilities for the quadratic function of Case 3. . . . . . 86

A.18 Coverage probabilities for the Weibull function of Case 3. . . . . . . . 87

A.19 Quadratic fit for Case 4 with $\lambda=8 \ldots \ldots \ldots$. . . . . . . . 88

A.20 Weibull fit for Case 4 with $\lambda=10000 \ldots \ldots$. . . . . . . . . . 89

A.21 Bias for the quadratic function of Case 4 . . . . . . . . . . . . . 89

A.22 Bias for the Weibull function of Case 4. We note that the graph is zoomed in significantly. . . . . . . . . . . . . . . . . . . 90

A.23 Coverage probabilities for the quadratic function of Case 4. . . . . . . 90

A.24 Coverage probabilities for the Weibull function of Case 4 . . . . . . . . . 91

A.25 Quadratic fit for Case 5 with $\lambda=10 \ldots \ldots$. . . . . . . . . . 92

A.26 Weibull fit for Case 5 with $\lambda=10000$. . . . . . . . . . . . . . . . . . 93

A.27 Bias for the quadratic function of Case 5 . . . . . . . . . . . . . . 93

A.28 Bias for the Weibull function of Case 5. We note that the graph is zoomed

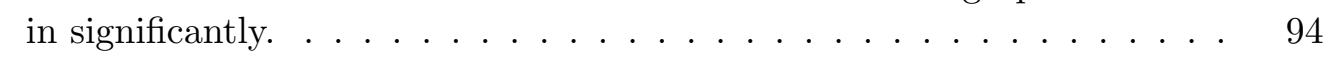

A.29 Coverage probabilities for the quadratic function of Case 5. . . . . . . . 94

A.30 Coverage probabilities for the Weibull function of Case 5 . . . . . . . . 95

A.31 Quadratic fit for Case 6 with $\lambda=15 \ldots \ldots \ldots$. . . . . . . . 96

A.32 Weibull fit for Case 6 with $\lambda=10000$. . . . . . . . . . . . . . . . . 97

A.33 Bias for the quadratic function of Case 6 . . . . . . . . . . . . . . 97

A.34 Bias for the Weibull function of Case 6. We note that the graph is zoomed in significantly. . . . . . . . . . . . . . . . . 98

A.35 Coverage probabilities for the quadratic function of Case 6. . . . . . . 98

A.36 Coverage probabilities for the Weibull function of Case 6. . . . . . . . . 99

A.37 Quadratic fit for Case 7 with $\lambda=4 \ldots \ldots$. . . . . . . . . . . 100

A.38 Weibull fit for Case 7 with $\lambda=10000$. . . . . . . . . . . . . . . . . . . 101

A.39 Bias for the quadratic function of Case 7. . . . . . . . . . . . . . . . 101

A.40 Bias for the Weibull function of Case 7. We note that the graph is zoomed in significantly. . . . . . . . . . . . . . . . . . 102

A.41 Coverage probabilities for quadratic function of Case 7 . . . . . . . . . . 102

A.42 Coverage probabilities for the Weibull function of Case 7. . . . . . . . . 103

A.43 Quadratic fit for Case 8 with $\lambda=6$. . . . . . . . . . . . . . . . 104

A.44 Weibull fit for Case 8 with $\lambda=10000$. . . . . . . . . . . . . . . . . 105

A.45 Bias for the quadratic function of Case 8 . . . . . . . . . . . . . 105

A.46 Bias for the Weibull function of Case 8 . We note that the graph is zoomed

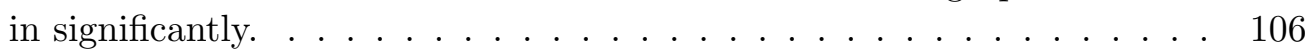

A.47 Coverage probabilities for the quadratic function of Case 8. . . . . . 106

A.48 Coverage probabilities for the Weibull function of Case 8. . . . . . . . . 107 
A.49 Quadratic fit for Case 9 with $\lambda=9 \ldots \ldots \ldots$. . . . . . . . 108

A.50 Weibull fit for Case 9 with $\lambda=10000$. . . . . . . . . . . . . . . . . . . 109

A.51 Bias for the quadratic function of Case 9 . . . . . . . . . . . . . 109

A.52 Bias for the Weibull function of Case 9. We note that the graph is zoomed

in significantly. . . . . . . . . . . . . . . 110

A.53 Coverage probabilities for the quadratic function of Case 9 . . . . . . . 110

A.54 Coverage probabilities for the Weibull function of Case 9 . . . . . . . . . 111

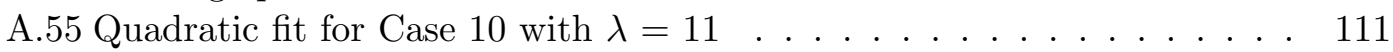

A.56 Bias for the quadratic function of Case 10 . . . . . . . . . . . . . 112

A.57 Coverage probabilities for the quadratic function of Case 10 . . . . . . 112

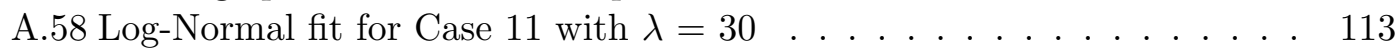

A.59 Bias for the log-normal function of Case $11 \ldots$. . . . . . . . . . . . . 114

A.60 Coverage probabilities for the log-normal function of Case 11 . . . . . . 114 


\section{List of Abbreviations}

CAV - Cardiac Allograph Vasculopathy

OLS - Ordinary Least Squares

RSS - Residual Sum of Squares

edf - Effective Degrees of Freedom

ODE - Ordinary Differential Equation

MLE - Maximum Likelihood Estimator

pdf - Probability Density Function

GCV - Generalized Cross-Validation

MSE - Mean Squared Error

REML - Restricted Maximum Likelihood 


\section{Introduction}

A clinical trial is defined by Friedman et al. as a study comparing the effect and value of intervention(s) against a control in human beings [1]. In the case of some clinical trials, data are said to follow a panel structure. Panel data are used in multiple disciplines, not only medicine, and refers to data that has been aggregated over intervals of time, or panels. The data may consist of counts of a specific occurrence, a count of state transitions, or another measure at a set of discrete time points. The exact times of the event in question remains unknown [2]. Modeling this type of data is often an interesting problem, as the belief of a continuous underlying structure gives way to a much larger possibility of function structures than the discrete case. Logically, the more follow-up times available, as well as how frequent these follow ups occur can potentially influence the fit of a continuous model and its usefulness. Another key feature of panel data is that observations from the same individual are correlated, giving way to patterns between individuals, but also adding a degree of complexity to the model [2]. An effective, low variation model catered to the specific structure of the data is often important to understand the data and find usefulness in predictive measures. Furthermore, since variability can be accounted for between individuals as well as within an individual's own observations, estimates of parameters (as well as their errors) are not skewed by the similarities in observations coming from the same individual [2]. However, they would be if this source of variation were not accounted for.

The purpose of this thesis is to identify under which scenarios a specific continuous penalized spline model applied to a Markov (or panel) structure may provide a suitable model fit. Several simulations under different conditions will be run to identify trends of increased model fit.

It is important to note that models of the structure to be developed in this thesis are commonly present in health studies of chronic and long lasting conditions. The followup times are not event driven, such as having a medical visit when a bone is broken or when a seizure occurs. The data has a predefined list of follow-up times and despite 
the events that may occur between visits to a medical professional, status is updated only at these predetermined times. Some examples of data of this nature include youth smoking status at various time points following the distribution of educational material about smoking (see Kalbfleish and Lawless, 1985) or a patient's level of cardiac allograph vasculopathy $(\mathrm{CAV})$ in the time following a heart transplantation (see Titman, 2011) [3. While significant analysis has been conducted to find stable models for panel data, the combination of a Markov structure applied to a penalized spline with a modelbased penalty has not been explored fully in previous studies. Titman (2011) explores the possibility of smooth transition intensities in a non-homogeneous Markov model [3], while Eliers and Marx investigate smoothing using both splines and penalties for additive Gaussian models [4]. This thesis will expand upon the ideas presented in both papers in the hopes of improving the transition intensity structure to more adequately model the panel data through the use of penalized splines, as well as allowing a parametrized model-based penalty term to drive the model should an underlying known parametric model fit the data more appropriately. These scenarios will be explored through a simulation study to investigate the statistical properties of the proposed estimators.

In Chapter 2, full background knowledge of basis and penalized splines and how they are used in modeling will be explained. Also, the Markov assumption and its associated structures will be explained and then linked to the context of the model in Chapter 3. In Chapter 4, the methods behind likelihood estimation using the Newton-Raphson method will be explored. Next, the model itself will be derived using the knowledge presented in Chapter 5. With this knowledge, the reader should be comfortable understanding the main ideas. Then, in Chapter 6, the simulation study will be presented. Numerous cases will be compared and discussed, in the hopes of providing a recommendation for the most appropriate use of the model in Chapter 7. Also, various statistics will be analyzed in order to achieve a recommendation for best practices in the use of the model presented. Lastly, a brief summary of the project and future recommendations will be discussed as a conclusion in Chapter 8. 


\section{$2 \quad$ Modeling Using Splines}

Preliminary definitions, as well as useful concepts, will be explained in this section in order to set the stage for the main component of the research.

\section{$2.1 \quad$ B-Splines}

Smoothing plays an important role in statistics today and has become a increasingly popular method for analyzing complex data as an alternative to parametric modeling. Various smoothing techniques exist, including kernel smoothers, splines and LOWESS among others [4]. In this thesis we consider basis splines (B-splines) as a smoothing method, though each variety has its own benefits.

Definition 1: A basis is defined as a set of linearly independent vectors. Any element of a vector space with a basis can be written as a linear combination of elements of the basis.

A substantial amount of research into B-Splines can be accredited to C. de Boor and his work in the 1970's [4. B-Splines are very useful for building non parametric models since their concept is simple and easily implemented. B-Splines have many desirable properties, such as the fact that they can be computed easily and they are numerically stable. However, B-Splines are dependent on knots, which require the modeler to determine the best number and position of these knots. This is a difficult task, without a clearly defined optimal solution for many cases. Over-fitting occurs with the use of too many knots, such as a knot at each data point, while too small of a number of knots results in over smoothing leading to a poor model fit [4].

B-splines of degree $q$ are formed using polynomial pieces of degree $q$ joined smoothly at a series of $n$ predefined interior knots and 2 boundary knots. This gives a total of $n+2$ knots. These polynomials, or basis functions are then multiplied by coefficient values. An algorithm for the computation of any B-Spline (once its knots have been defined) was introduced by de Boor in 1978 [5]. Oftentimes, a total number of knots 
less than the number of data points is used, along with a penalty term to account for over-fitting. This process will be discussed further in later chapters.

\subsubsection{Properties of B-Splines}

We assume we are working with B-Splines of degree $q[4]$. Then we have a series of basis functions that adhere to the following properties:

1. A spline basis of degree $q$ has $q+1$ polynomial pieces, each of degree $q$.

2. The polynomial pieces are joined smoothly at $q$ inner knots (this does not include end points) by forcing them and up to the $q-1^{\text {th }}$ derivatives to match

3. At every point $x$, continuous derivatives up to degree $q-1$ exist

4. Each basis function is positive on a domain spanned by $q+2$ knots; everywhere else it is zero

5. At a point $x, q+1$ basis functions are non zero

To better illustrate the above properties, we consider Figure 1, found originally in Elier and Marx's 1996 paper. We can clearly see the changes in the B-spline when comparing a one degree versus two degree spline bases, as well as how overlapping spline bases are visualized.

Finally, it is important to note that both derivatives and integrals of B-Splines can be calculated relatively easily, and this becomes necessary when the B-Splines are penalized [4].

\subsubsection{Regression with B-Splines}

The purpose of ordinary least squares regression (OLS) is to model a set of observed points, $y_{i}, i=1, \ldots, n$ as a function of $p$ variables and their coefficients, $\beta_{1}, \ldots \beta_{p}$. Model fit is assessed using the principle of least squares. Least squares is used to minimize the distance between the predicted $y$ value, $\hat{y}_{i}$, and the observed $y$ value, $y_{i}$. This distance is 

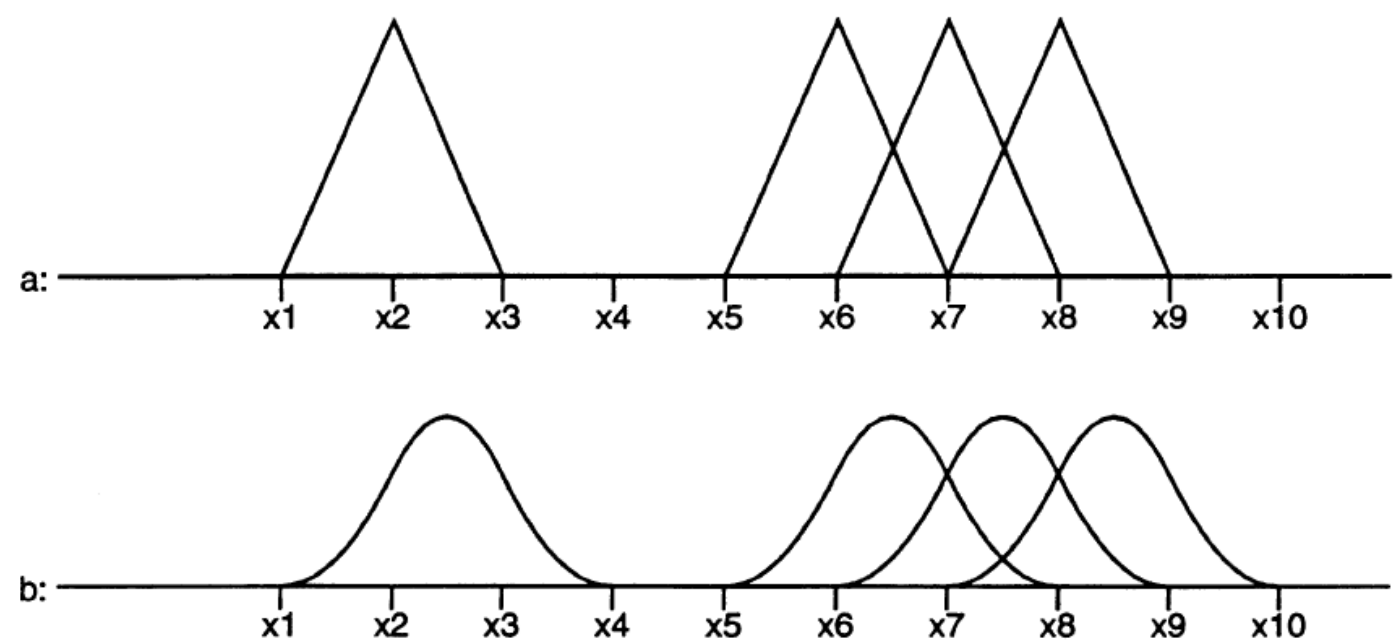

Figure 1: A visual representation of 1(a) and 2(b) degree B-Splines basis functions, as well as their overlapping patterns [4].

known as a residual, and OLS models minimize the square of the residuals. Other forms of regression use different criteria to obtain model fits, such as the likelihood function.

Using the same principles as ordinary least squares regression (OLS), one can model a set of data points using basis splines (B-splines). In this section, we explain the principles behind using polynomial splines for modeling, and how the typical OLS model is modified with the use of basis functions.

We begin by considering a set of data points $\left(y_{i}, x_{i}\right), i=1, \ldots, n$, with $x$ values defined in the interval $[l, u]$ and $y_{i}$ 's independently distributed $N\left(f\left(x_{i}\right), \sigma^{2}\right)$ variables. Suppose we wish to model these points using a function $f(x)$ and an error measurement $\epsilon(x) . \epsilon(x)$ is assumed to be from a normal distribution with mean 0 and variance $\sigma^{2}$. We write this model as

$$
y(x)=f(x)+\epsilon(x)
$$

We are now interested in the structure of our $f(x)$ function. In OLS, $f(x)$ is presented as $\mathbf{X} \beta$, where $\mathbf{X}$ is a design matrix (a matrix of values of the explanatory variables for each of the $n$ observations) of dimension $n$ by $p$, where $n$ is the number of data points and $p$ is the number of unknown parameters in our function. Here $\beta$ is a $p$ by 1 vector 
of unknown parameters. Thus we can write

$$
\mathbf{y}=\mathbf{X} \beta+\epsilon .
$$

where $\mathbf{y}$ and $\epsilon$ are $n \times 1$ vectors of the observed values and their associated errors respectively. For a single observation, this equation expands to give

$$
y_{i}=\beta_{0} x_{0 i}+\beta_{1} x_{1 i}+\ldots+\beta_{p} x_{p i}+\epsilon_{i}
$$

as the equation of the observed data point $y_{i}=y\left(x_{i}\right)$.

With B-splines, it is possible to obtain an equation of similar structure. First we assume that $f \in \mathbf{S}_{d, k}$ where $\mathbf{S}_{d, k}$ is the space of splines of degree $d$ with $k$ inner knots. Then, a series of basis functions can represent $\mathbf{S}_{d, k}$. The space $\mathbf{S}_{d, k}$ is spanned by $d+k+1$ basis functions denoted by

$$
\left\{B_{0}(t), B_{1}(t), B_{2}(t), B_{3}(t), \ldots, B_{d+k}(t)\right\}
$$

For example, if $f(x)$ is a cubic spline with two knots it can be represented as

$$
f(x)=\sum_{j=0}^{5} u_{j} B_{j}(x) .
$$

Figure 2 demonstrates an example of such a B-Spline function and its basis functions multiplied by corresponding coefficients to represent a function. We note that the underlying basis functions vary in amplitude depending on the coefficient values.

This definition gives us the ability to write the observations as

$$
y_{i}=y\left(x_{i}\right)=\sum_{j=0}^{d+k} u_{j} B_{j}\left(x_{i}\right)+\epsilon_{i} .
$$

In matrix notation, we can write this as

$$
\mathbf{y}=\underline{\mathbf{B}} u+\epsilon
$$




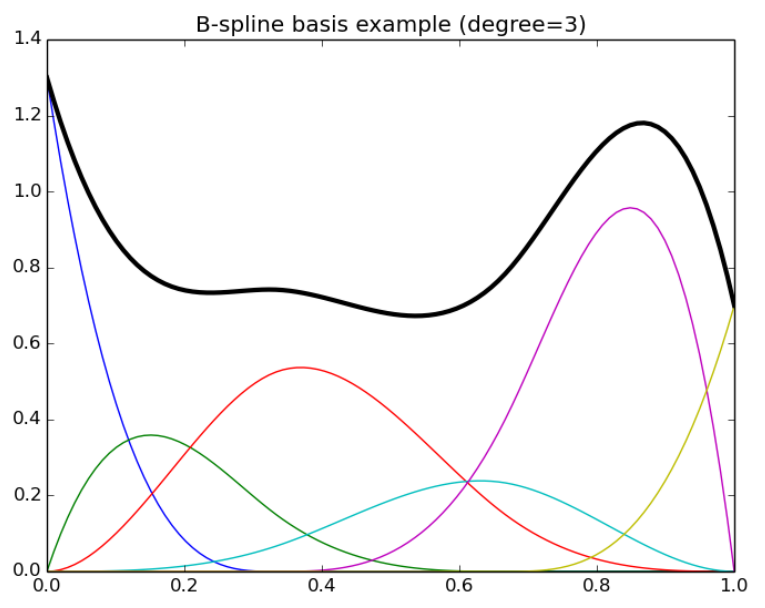

Figure 2: A B-Spline resulting from coefficients multiplying the cubic basis functions spanning the linear space to which the function is assumed to belong.

where $y$ is an $n$ by 1 vector of observed $y$ values, $\mathbf{B}$ is an $n$ by $d+k+1$ matrix where each row represents the values of the basis functions at the $i$ th observed $x$ value, $u$ is a $d+k+1$ by 1 vector of unknown parameters and $\epsilon$ is an $n$ by 1 vector of error terms.

OLS is built upon the fact that the $\hat{\beta}$ estimates, or in the B-spline case, the $\hat{u}$ estimates, minimize the residual sum of squares (RSS). The equation for the RSS is given by

$$
\begin{aligned}
R S S(f) & =\sum_{i=1}^{n}\left(y_{i}-f\left(x_{i}\right)\right)^{2} \\
& =\sum_{i=1}^{n}\left\{y_{i}-\sum_{j=1}^{d+k} u_{j} B_{j}\left(x_{i}\right)\right\}^{2}
\end{aligned}
$$

where $n$ is the total number of data points.

In OLS, we solve for our $\hat{\beta}$ estimator by finding a set of normal equations using least squares estimation that give

$$
\hat{\beta}=\left(\mathbf{X}^{T} \mathbf{X}\right)^{-1} \mathbf{X}^{T} \mathbf{y}
$$

Similarly, when using B-Splines, we know that estimates for our unknown parameters $u$ 
can be obtained by solving $\hat{u}=\left(\mathbf{B}^{T} \mathbf{B}\right)^{-1} \mathbf{B}^{T} \mathbf{y}$. As a result, we can obtain an estimated fit for the data $\widehat{f(x)}$ given by

$$
\widehat{f(x)}=\mathbf{B} \hat{u}=\mathbf{B}\left(\mathbf{B}^{T} \mathbf{B}\right)^{-1} \mathbf{B}^{T} \mathbf{y}
$$

Though estimating parameters is relatively simple, configuring $\mathbf{B}$ requires specifying the degree of the B-spline and the number and location of knots. Cubic splines are most often used as they provide enough flexibility without being too erratic. Increasing the number of knots reduces any smoothing effect and can lead to interpolation of the data. One way to solve the problem of placing too many knots is to impose a penalty term which controls the oscillation of the estimated function. We now discuss this approach in more detail.

\section{$2.2 \quad$ Penalized Splines}

Penalized splines require the addition of a penalty function and parameter, $\lambda$, to the spline model discussed in the previous section. If we consider the space of functions with two absolutely continuous derivatives, the best model fit for a set of data will be one that minimizes the now penalized residual sum of squares. The RSS equation now becomes

$$
\begin{aligned}
R S S(f) & =\sum_{i=1}^{n}\left(y_{i}-f\left(x_{i}\right)\right)^{2}+\lambda \int f^{\prime \prime}(v)^{2} d v \\
& =\sum_{i=1}^{n}\left\{y_{i}-\sum_{j=0}^{d+k} u_{j} B_{j}\left(x_{i}\right)\right\}^{2}+\lambda \int\left\{\sum_{j=0}^{d+k} u_{j} B_{j}^{\prime \prime}(v)\right\}^{2} d v
\end{aligned}
$$

where $\lambda$ is a fixed smoothing parameter. In matrix notation we can write

$$
R S S=(\mathbf{Y}-\mathbf{B} \underline{u})^{T}(\mathbf{Y}-\mathbf{B} \underline{u})+\lambda \underline{u}^{T} \mathbf{P} \underline{u}
$$

where $\mathbf{P}=D_{2}^{T} D_{2}$, if the second order divided difference penalty is used [4]. $\mathbf{P}$ can be the exact second derivative term or an approximation, such as a divided difference. In 
the B-Spline case, the exact penalty is given by

$$
\mathbf{P}=\left\{P_{i j}\right\}=\left\{\int B_{i}^{\prime \prime}(t) B_{j}^{\prime \prime}(t) d t\right\}
$$

The addition of a penalty term in fact penalizes the coefficients of the spline, causing them to shrink towards a linear fit [6], the solution to the differential equation $\mathbf{D}^{2} \mathbf{f}=0$. The first term effectively measures how close the estimated function is to the data, while the second term penalizes the curvature in the estimated function. Then $\lambda$ serves as a trade off parameter between the two. As $\lambda$ approaches 0 , the function $\hat{f}$ fits the data more closely. Alternatively, as $\lambda$ approaches $\infty$, the function approaches a line, since no second derivative can be tolerated [6].

Now, in order to solve for $\underline{u}$, we take the first derivative of the above and set it to zero, that is

$$
-2 \mathbf{B}^{T}(\mathbf{Y}-\mathbf{B} \underline{u})+2 \lambda \mathbf{P} \underline{u}=0
$$

Solving for $\underline{u}$ we get

$$
\underline{\hat{u}}=\left(\mathbf{B}^{T} \mathbf{B}+\lambda \mathbf{P}\right)^{-1} \mathbf{B}^{T} \mathbf{Y}
$$

which gives

$$
\begin{aligned}
\hat{\mathbf{f}} & =\mathbf{S Y} \\
& =\mathbf{B}\left(\mathbf{B}^{T} \mathbf{B}+\lambda \mathbf{P}\right)^{-1} \mathbf{B}^{T} \mathbf{Y} .
\end{aligned}
$$

We note that $\operatorname{trace}(\mathbf{S})$ gives the effective degrees of freedom, which is analogous to the trace of that hat matrix, $\operatorname{trace}(\mathbf{H})$ in OLS. The effective degrees of freedom, or edf, does not have to be integer valued.

As shown above, the second derivative penalty is not the only option as a penalty function. Different orders of derivatives can also be used, with higher order derivatives 
leading to smoother fits [4]. In their 1996 paper on flexible smoothing with B-Splines and Penalties, Eliers and Marx even propose using a penalty term based on divided differences to simplify calculations, as no higher order derivative terms need to be calculated. Their proposition gives the RSS equation

$$
R S S(f)=\sum_{i=1}^{n}\left\{y_{i}-\sum_{j=0}^{d+k} u_{j} B_{j}\left(x_{i}\right)\right\}^{2}+\lambda \sum_{j=c+1}^{d+k}\left(\Delta^{k} u_{j}\right)^{2}
$$

where $c$ is the order of the derivative we are approximating with divided differences [4].

\subsubsection{Derivatives of B-Splines}

A recursive relationship for calculating B-Splines exists and is numerically efficient [5]. In 1978, de Boor derived that for a basis function of degree $q$ with $2 q$ exterior knots given by $\xi_{h}, h=-q, \ldots, q+k$, where $k$ is the number of fixed interior knots, we have

$$
B_{d, h}=\frac{t-\xi_{h}}{\xi_{h+d}-\xi_{h}} B_{d-1, h}(t)+\frac{\xi_{h+d+1}-t}{\xi_{h+d+1}-\xi_{h+1}} B_{d-1, h+1}(t) .
$$

However, a useful approximation to the derivatives of B-Splines is relatively simple to compute. In fact, de Boor (1978) showed that an approximation to derivatives of a B-Spline of degree $q, B_{j}(x ; q)$ can be calculated as follows:

$$
\begin{aligned}
h \sum_{j} a_{j} B_{j}^{\prime}(x ; q) & =\sum_{j} B_{j}(x ; q-1)-\sum_{j} a_{j+1} B_{j+1}(x ; q-1) \\
& =-\sum_{j} \Delta a_{j+1} B_{j}(x ; q-1)
\end{aligned}
$$

for the first derivative [4]. Here $h$ is the distance between knots and $\Delta a_{j}=a_{j}-a_{j-1}$. Then for the second (and higher order) derivatives, we use induction to get

$$
h^{2} \sum_{j} a_{j} B_{j}^{\prime \prime}(x ; q)=\sum_{j} \Delta^{2} a_{j} B_{j}(x ; q-2)
$$

as the second derivative. Here $\Delta^{2}=\Delta \Delta a_{j}=a_{j}-2 a_{j-1}+a_{j-2}[4]$. 


\subsubsection{The Penalty Term}

In most cases, a second derivative penalty (or an approximation to the second derivative) is considered to be effective for a wide variety of functions. That is, all functions with absolutely continuous second derivatives. A second derivative penalty is known to work very well to avoid overfitting and increase smoothness [4, thus an approximation of the second derivative given by divided differences is often the penalty of choice in many scenarios. While this is an attractive approach, there is also an argument to be made for using a specific function as the penalty term.

\subsubsection{Model-Based Penalized Splines}

Using a model-based penalty allows for a greater class of functions to be penalized, including those with continuous second derivatives as well as those without continuous second derivatives. Also, it allows for any apriori assumptions of a distribution to be used advantageously. For example, if the data being analyzed are known to come from a lognormal distribution, a model-based penalty using a log-normal probability distribution function will ultimately fit the data more successfully than a penalty that only relies on the second derivative of the function. Thus, if used correctly and appropriately, a model-based penalty can reduce the standard error of the model. This is due to the fact that the model-based penalty term often uses maximum likelihood estimates for its parameters, which by their definition produce the smallest variance and bias out of all possible estimators for a parameter [13].

Heckman and Ramsay consider the alternate possibility of a different linear operator as a penalty term in their papers on the topic in 1997 and 2000 [ 9 . According to Heckman and Ramsay, the standard second derivative penalty of the function tends to favour a standard linear model for the data 8 . By allowing the penalty term to differ using a differential linear operator, the penalty term becomes more flexible. Also, a predefined understanding of the context of the data obtained can be used to narrow down a potential linear operator that would best suit the situation. For example, Heckman 
and Ramsay consider using a periodic linear differential operator as a penalty, with solution

$$
\alpha_{0}+\alpha_{1} t+\alpha_{3} \cos (\omega t)+a_{4} \sin (\omega t)
$$

where the penalty is parametrized by $\omega$, which is initially estimated from the data. The linear differential operator in this case is given by $\left(\mathbf{D}^{4}+\omega \mathbf{D}^{2}\right) \mathbf{f}=0$, which has a null space $\{1, t, \cos (\omega t), \sin (\omega t)\}$.

As shown in Figure 3, this penalty allows the model to capture both a periodic and linear trend in data regarding melanoma incidences. Melanoma of course is periodic in the sense that more cases occur during seasonal exposure to sunlight [8].

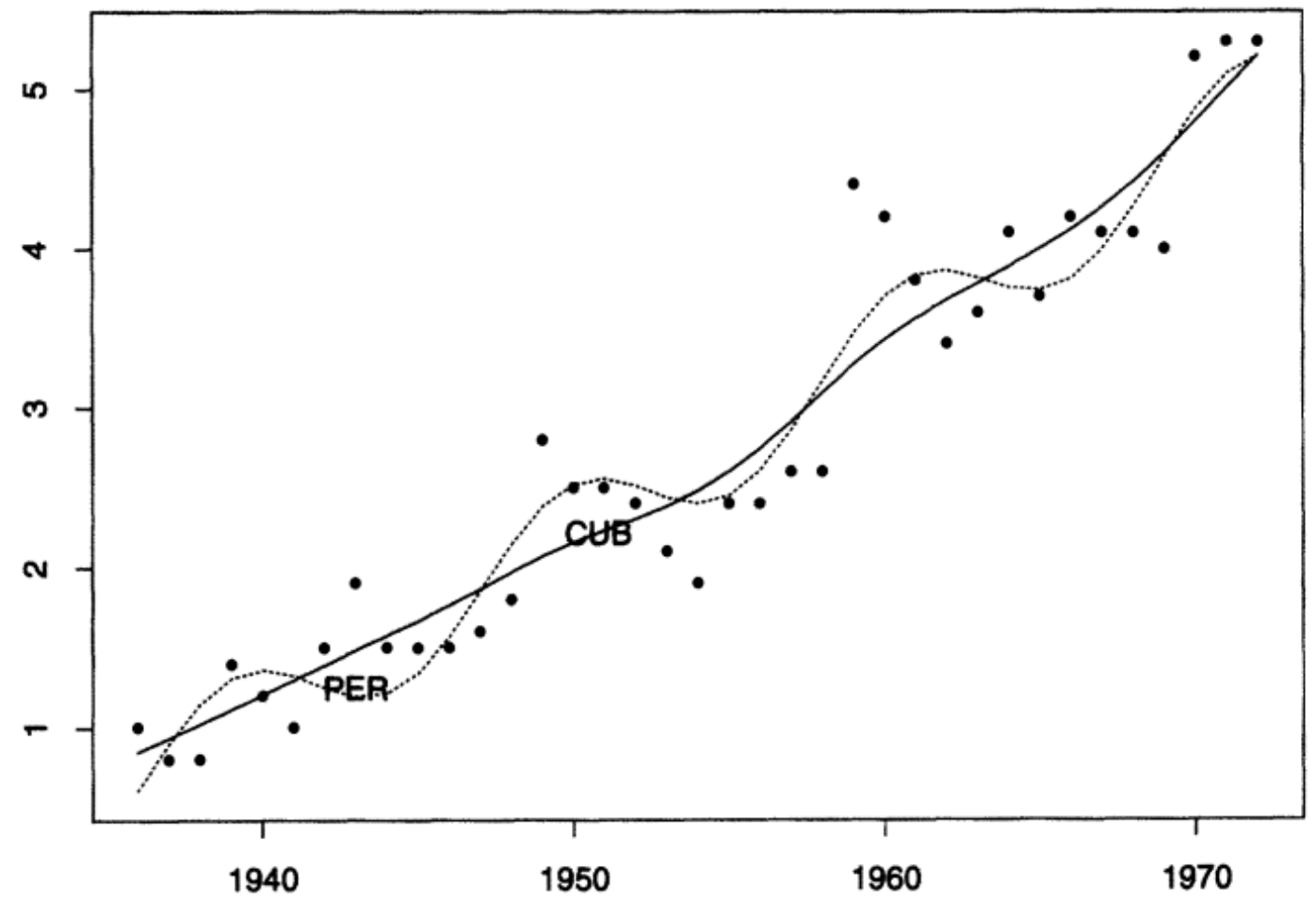

Figure 3: Two models for melanoma data. The CUR model includes the standard second derivative penalty, while the PER model uses a periodic parametrized penalty [8].

In health studies with panel data, multiple different model-based penalties can be considered. We can consider penalties based on PDEs, ODEs or intensity functions for example. However, when using ODEs and PDEs, we must have consistent estimators 
for any parameters in the equations, such as the $\omega$ in the example above.

In the model presented in this thesis, we will consider the hazard function of the Weibull distribution with two parameters, for which we obtain consistent estimators. The hazard function of the Weibull distribution is a popular parametric choice in health studies [10] [14]. The idea here is that if the data demonstrates an underlying Weibull intensity function, the model will be fit with an inflated penalty parameter $\lambda$, forcing the model fit to lean towards a true Weibull intensity. Conversely, when the underlying function of the data does not somewhat follow a Weibull intensity, the $\lambda$ parameter will be shrunk in order to minimize the effect of the penalty term.

The parameter estimates, derivatives and likelihood of the Weibull intensity will be derived in subsequent chapters At this point, it is only essential to understand that a variety of functions can be used in the penalty term and that in some cases the most appropriate penalty function can be deduced by understanding the context of the data being analyzed. Thus, depending on the nature of the data, different penalty functions may be more appropriate depending on apriori knowledge. Furthermore, it is possible that a different penalty function would be most appropriate to different subsets of data. That is, we are not limited to a single penalty function for an entire data set.

Next, we consider data analysis under a Markov assumption and identify possible ways to include B-Splines and penalties while modeling in this context. 


\section{The Markov Assumption}

This section describes the basic structure of a Markov process and some useful properties that will be of key interest when modeling panel data under this assumption.

\subsection{The Markov Process}

Named after Andrey Markov, a Russian mathematician, the concept of a Markov process has proven useful in many domains[11]. Markov processes are frequently used in various scientific problems as well as for health data.

Suppose there exists a continuous-time stochastic process with $k$ possible states. We denote this process by $\{X(t), t \epsilon(0, \infty)\}$, where at each time point the process takes on a state from $S=\{1,2, \ldots, k\}$.

Definition 2: A collection of data are said to be a Markov chain if for $0 \leq s \leq t$,

$$
P\left\{X(t)=j \mid X(s)=i, \mathcal{H}\left(s^{-}\right)\right\}=P\{X(t)=j \mid X(s)=i\} \forall i, j .
$$

The history of the process is denoted by $\mathcal{H}=\{X(u), 0 \leq u \leq s\}$.

This definition implies that the conditional distribution of a future state, given any previous states as well as the current state, is only dependent on the current state. This is known as the Markov property [11]. It implies that modeling future states requires only the knowledge of the last. Thus, it is logical to consider the probability of progressing from the current state to any other possible future state.

Definition 3: Let $X(t)$ be a continuous -time Markov process. Then $p_{i j}(s, t)=P\{X(t)=$ $j \mid X(s)=i\} \forall i, j$ is the transition probability of the process starting from state $i$ at time $s$ to state $j$ at time $t, 0 \leq s \leq t$.

We can write these probabilities concisely using matrix notation, which gives a $k \times k$ matrix $\mathbf{P}(s, t)=\left\{p_{i j}(s, t)\right\}$. We restrict $\mathbf{P}$ so that $p_{i j}(s, t) \geq 0$ and $\sum_{j} p_{i j}(s, t)=$ 
1. Lastly, we define properties of the Markov process using what are known as the Chapman-Kolmogorov equations. We write these equations as

$$
p_{i j}(s, t)=\sum_{k \epsilon S} p_{i k}(s, u) p_{k j}(u, t) \forall i, j .
$$

which in matrix notation can be expressed as

$$
\mathbf{P}(s, t)=\mathbf{P}(s, u) \mathbf{P}(u, t)
$$

for $0 \leq s \leq u \leq t$

\subsubsection{The Transition Intensity Matrix}

We now consider derivatives of $\mathbf{P}$.

Definition 5: We define the transition intensity matrix or infinitesimal generator matrix $\mathbf{Q}(\mathbf{t})$ of a Markov process as

$$
\mathbf{Q}=\left\{q_{i j}(t)\right\}_{K \times K}
$$

where the individual elements of the transition intensity matrix representing the instantaneous transition probabilities from state $i$ to state $j$ are [1]

$$
\begin{aligned}
q_{i j}(t) & =\lim _{\epsilon \rightarrow 0^{+}} \frac{p_{i j}(\epsilon)}{\epsilon} \\
& =\lim _{\epsilon \rightarrow 0^{+}} \frac{P\{X(t+\epsilon)=j \mid X(t)=i\}}{\epsilon}
\end{aligned}
$$

and the matrix $\mathbf{Q}(t)$ thus becomes

$$
\mathbf{Q}(t)= \begin{cases}q_{i j}(t) & , i \neq j \\ -\sum_{j \neq i} q_{i j}(t) & , i=j .\end{cases}
$$


There is a important relationship between $\mathbf{P}(s, t)$ and $\mathbf{Q}(t)$ called the forward and reverse Chapman-Kolmogorov equations. The forward equation is given by

$$
\frac{\partial}{\partial t} \mathbf{P}(s, t)=\mathbf{P}(s, t) \mathbf{Q}
$$

and the reverse by

$$
\frac{\partial}{\partial s} \mathbf{P}(s, t)=\mathbf{Q}(s) \mathbf{P}(s, t)
$$

which are a system of ordinary differential equations (ODEs). Denoting $\mathbf{P}(t)=\mathbf{P}(0, t)$ without loss of generality and assuming the initial condition $\mathbf{P}(0)=\mathbf{I}$ and $\mathbf{Q}(u)=\mathbf{Q}$ on $u \in[0, t]$ the Chapman-Kolmogorov forward equation has the following solution:

$$
\begin{aligned}
\mathbf{P}(t) & =e^{\mathbf{Q}(t)} \\
& =\sum_{s=0}^{\infty} \frac{\mathbf{Q}^{s} t^{s}}{s !} .
\end{aligned}
$$

\subsubsection{The Decomposition Method}

In order to more easily work with the $\mathbf{Q}$ matrix, we use an eigenvalue decomposition to simplify our previous expressions. We first consider:

$$
\begin{aligned}
\mathbf{Q E} & =\left[Q e_{1} \ldots Q e_{K}\right] \\
& =\left[e_{1} \lambda_{1} \ldots e_{K} \lambda_{K}\right] \\
& =\mathbf{E} \boldsymbol{\Lambda}
\end{aligned}
$$

where $\boldsymbol{\Lambda}=\operatorname{diag}\left\{\lambda_{1}, \ldots, \lambda_{K}\right\}$, with $\lambda_{K}$ and $e_{K}$ representing corresponding eigenvalue and eigenvector of $\mathbf{Q}$. As long as $\mathbf{E}$ has $K$ linearly independent eigenvectors, we can rewrite our expression for $\mathbf{Q}$ as

$$
\mathbf{Q}=\mathbf{E} \Lambda \mathbf{E}^{-1}
$$


We now apply this to our expression for $\mathbf{P}(t)$ to get a more simplified result to be used in computations.

$$
\begin{aligned}
\mathbf{P}(t) & =e^{\mathbf{Q} t} \\
& =\sum_{s=0}^{\infty} \frac{\mathbf{Q}^{s} t^{s}}{s !} \\
& =\sum_{s=0}^{\infty} \frac{\left(\mathbf{E} \boldsymbol{\Lambda} \mathbf{E}^{-\mathbf{1}}\right)^{s}}{s !} \\
& =\sum_{s=0}^{\infty} \frac{\mathbf{E} \boldsymbol{\Lambda}^{\mathbf{s}} \mathbf{E}^{-\mathbf{1}}}{s !} \\
& =\mathbf{E}\left(\sum_{s=0}^{\infty} \operatorname{diag}\left\{\frac{\lambda_{1}^{s} t^{s}}{s !}, \ldots, \frac{\lambda_{K}^{s} t^{s}}{s !}\right\}\right) \mathbf{E}^{-1} \\
& =\mathbf{E} \operatorname{diag}\left\{\sum_{s=0}^{\infty} \frac{\lambda_{1}^{s} t^{s}}{s !}, \ldots, \frac{\lambda_{K}^{s} t^{s}}{s !}\right\} \mathbf{E}^{-1} \\
& =\mathbf{E} \operatorname{diag}\left\{e^{\lambda_{1} t}, \ldots, e^{\lambda_{K} t}\right\} \mathbf{E}^{-1}
\end{aligned}
$$

Note that the elements of $\mathbf{E}$ and of $\lambda_{i}$ are functions of the elements of $\mathbf{Q}$. This decomposition will become useful during the computational aspect of the model to be presented later in this thesis as it simplifies the numerical computations.

When $\mathbf{Q}$ is a constant, our Markov chain is homogeneous. However, what is truly of interest here is the non-homogenous case. We will explore this more thoroughly in subsequent chapters, but first it is necessary to understand the main method of inference used in modeling panel data under a Markov assumption: maximum likelihood estimation and the Newton-Raphson method. 


\section{Likelihood Analysis and Parameter Estimation}

In this section we explore the likelihood function and its properties. We consider them in the general case, and work through the Newton-Raphson algorithm for parameter estimation.

\subsection{Likelihood Analysis}

The likelihood function is arguably one of the most widely used statistics [13]. We begin with a formal definition.

Definition 4: Let $f(\mathbf{x} \mid \Psi)$ denote the joint probability distribution function of a sample of data $\mathbf{X}=X_{1}, \ldots, X_{n}$. Then, given that $\mathbf{X}=\mathbf{x}$ is observed, the function of $\Psi$ defined by

$$
\mathcal{L}(\Psi \mid \mathbf{x})=f(\mathbf{x} \mid \Psi)
$$

is called the likelihood function where $\Psi$ is a vector of parameters of interest.

In order to apply the likelihood function to panel structure data, we must consider a sample size of $n$ individuals, followed in a study for a length of time $T$. We assume that the history of each person in the study can be described using a non-homogeneous continuous time Markov process with transition intensity matrix $\mathbf{Q}(t ; \underline{\Psi})$ where $\underline{\Psi}$ is a vector of parameters that defines $\mathbf{Q}$ and $t \in[0, T]$. We have information about individuals at a set of specific time points $\tau=\left\{t_{0}, \ldots, t_{m}\right\}$ where $t_{0}=0, t_{m}=T$ and $t_{0} \leq t_{1} \leq \cdots \leq t_{m}$. The data then consists of the state of each individual at each time point in $\tau$. For the likelihood function, we then consider how many individuals, say $n_{i j l}$, were in state $i$ at time $t_{l-1}$ and state $j$ at time $t_{l}$ out of the $k$ possible states. The likelihood function for panel data under a homogeneous continuous-time Markov assumption is given by

$$
\mathcal{L}(\underline{\Psi})=\prod_{l=1}^{m} \prod_{i=1}^{k} \prod_{j=1}^{k} p_{i j}\left(t_{l-1}, t_{l} ; \underline{\Psi}\right)^{n_{i j l}}
$$


where $p_{i j}\left(t_{l-1}, t_{l} ; \underline{\Psi}\right)$ denotes the conditional probability of moving from state $i$ at time $t_{l-1}$ to state $j$ at time $t_{l}$.

In order to determine estimates for the parameter $\underline{\Psi}$, a maximum likelihood procedure is followed. However, in order to simplify calculations, we instead maximize the log-likelihood. Thus we have

$$
\log \mathcal{L}(\underline{\Psi})=\sum_{l=1}^{m} \sum_{i=1}^{k} \sum_{j=1}^{k} n_{i j l} \log \left(p_{i j}\left(t_{l-1}, t_{l} ; \underline{\Psi}\right)\right) .
$$

We maximize this equation by solving $\frac{\partial}{\partial \underline{\Psi}} \log \mathcal{L}(\underline{\Psi})=0$. Since $\underline{\Psi}$ is a vector, we must maximize a system of equations. Here, we use what is called the Newton-Raphson method.

\subsection{Fisher's Scoring Method for Parameter Estimation}

Fisher's Scoring method replaces the Hessian of the log-likelihood function with the negative of the second partial derivatives. When the negative expectation of the second derivative partials is used for inference, the Hessian is replaced with Fisher's Information in the calculation. This is known as the Newton-Raphson method.

The Newton-Raphson method is one of the most widely used methods in statistics for parameter estimation [12]. The algorithm begins by using initial estimates for a set of parameters, say $\underline{\Psi}$ in our case. Then, we also require the first and second partial derivatives of the log-likelihood function above calculated using the initial estimate for the parameter. We refer to the first partials as the score function, and the expectation of the negative of the second partial derivatives as Fisher's Information.

The score function is used to maximize the likelihood function, and equivalently the log-likelihood function, since the derivative of a function will be zero at a maximum or 
minimum point [2]. Thus, the score function under a Markov assumption is given by

$$
\begin{aligned}
S(\underline{\Psi}) & =\frac{\partial}{\partial \underline{\Psi}} \log \mathcal{L}(\underline{\Psi}) \\
& =\frac{\partial}{\partial \underline{\Psi}} \sum_{l=1}^{m} \sum_{i=1}^{k} \sum_{j=1}^{k} n_{i j l} \log \left(p_{i j}\left(t_{l-1}, t_{l} ; \underline{\Psi}\right)\right)=\underline{0} .
\end{aligned}
$$

Unfortunately, the derivative of the probability function $\mathbf{P}(t ; \underline{\Psi})$ is not a straightforward calculation. A closed form expression is not easily obtained, so the diagonalization method discussed earlier will be used in order to obtain closed form expressions for both $\mathbf{P}(t ; \underline{\Psi})$ and its derivatives. In the next section when we construct the full model, this process will be shown in detail for the specific model used in the simulation study.

The next step in the Newton-Raphson method is to calculate Fisher's Information 2. Fisher's Information is defined as

$$
E\left(-\frac{\partial^{2} \log \mathcal{L}}{\partial \underline{\Psi} \partial \underline{\Psi}^{T}}\right)
$$

The inverse of this quantity is the asymptotic variance of the parameter $\underline{\Psi}$. Once these two derivative quantities are found, the initial values for our parameter $\underline{\Psi}^{(0)}$ are updated using the equation

$$
\underline{\Psi}^{(r+1)}=\underline{\Psi}^{(r)}+\left\{E\left(-\frac{\partial^{2} \log \mathcal{L}}{\partial \underline{\Psi} \partial \underline{\Psi}^{T}}\right)\right\}^{-1} S(\underline{\Psi})
$$

where $r$ is the iteration number. This iterative process is repeated until convergence is reached, which results in final values of $\Psi$ that we call the maximum likelihood estimator, $\underline{\hat{\Psi}}$ When samples are large, maximum likelihood estimates hold many convenient properties. For example, they are asymptotically unbiased estimators 2]. Furthermore, they are asymptotically efficient, meaning that their variance is the lowest of all possible variances of other estimators [2]. Thus, the resulting estimator is appropriate for use in many statistical tests and models.

We have now covered a sufficient amount of background to understand all of the 
components of the model to be presented in this thesis. In this next section, we construct the model, presenting all details and rationale in preparation for the simulation study. 


\section{Penalized Spline Model for Panel Data Under a Markov Assumption}

We will now describe in detail a model for panel data under a Markov assumption that incorporates all of the previously discussed topics. This model will be appropriate for panel data, yet more flexible than the fully parametric case.

Here we will assume the data given is a count of the number of transitions from one state to another out of $k=2$ possible states observed at the end of a particular time panel. We have a total of $l$ times and thus a total of $l-1$ time panels. We assume that the continuous time transition intensity matrix $\mathbf{Q}(t)$ has the form

$$
\mathbf{Q}(t)=\left[\begin{array}{cc}
-q_{12}(t) & q_{12}(t) \\
q_{21}(t) & -q_{21}(t)
\end{array}\right]
$$

The number of states can be increased as needed. For a $k$ state model the transition intensity will have the following form,

$$
\mathbf{Q}(t)=\left[\begin{array}{cccc}
-\sum_{i=2}^{k} q_{1 i}(t) & q_{12}(t) & \cdots & q_{1 k}(t) \\
q_{21}(t) & -\sum_{i=1,3}^{k} q_{2 i}(t) & \cdots & q_{2 k}(t) \\
\vdots & \vdots & \ddots & \vdots \\
q_{k 1}(t) & q_{k 2}(t) & \cdots & -\sum_{i=1}^{k-1} q_{k i}(t)
\end{array}\right]
$$

where the diagonal element is the negative of the sum of the off diagonal row elements.

In order to provide flexibility in the transition intensities, we need a flexible structure for each $q_{i j}(t)$ which is assumed to be in a space of smooth continuous functions with continuous second derivatives. Here we will represent each element of $\mathbf{Q}(\mathrm{t})$ as a spline.

$$
q_{i j}(t)=e^{\underline{B}(t)^{T}} \underline{\Psi}_{i j}
$$

where $B(t)$ is a vector of the $d+k$ B-spline functions evaluated at a time period $t$ and $\underline{\Psi}_{i j}$ are the parameters we wish to estimate for a transition from state $i$ to state $j$. 
Next we consider the expected number of transitions per time period per person, which is given by the integral of the $\mathbf{Q}(t)$ matrix given by

$$
\begin{aligned}
\mathbf{Z}\left(t_{l-1}, t_{l}\right) & =\int_{t_{l-1}}^{t_{l}} \mathbf{Q}(u) d u \\
& =\left\{\int_{t_{l-1}}^{t_{l}} q_{i j}(u) d u\right\}
\end{aligned}
$$

where $\left[t_{l-1}, t_{l}\right]$ is the time panel in question. Thus, $\mathbf{Z}\left(t_{l-1}, t_{l}\right)$ during a specified time panel in the two state case would be

$$
\mathbf{Z}\left(t_{l}, t_{l+1}\right)=\left[\begin{array}{cc}
-z_{12} & \int_{t_{l}}^{t_{l+1}} q_{12}(u) d u \\
\int_{t_{l}}^{t_{l+1}} q_{21}(u) d u & -z_{21}
\end{array}\right]
$$

and if in the 3 state case

$$
\mathbf{Z}\left(t_{l}, t_{l+1}\right)=\left[\begin{array}{ccc}
-\left(z_{12}+z_{13}\right) & \int_{t_{l}}^{t_{l+1}} q_{12}(u) d u & \int_{t_{l}}^{t_{l+1}} q_{13}(u) d u \\
\int_{t_{l}}^{t_{l+1}} q_{21}(u) d u & -\left(z_{21}+z_{23}\right) & \int_{t_{l}}^{t_{l+1}} q_{23}(u) d u \\
\int_{t_{l}}^{t_{l+1}} q_{31}(u) d u & \int_{t_{l}}^{t_{l+1}} q_{32}(u) d u & -\left(z_{31}+z_{32}\right)
\end{array}\right] .
$$

Next, we decompose $\mathbf{Z}$ into $\mathbf{E} \mathbf{\Lambda} \mathbf{E}^{-1}$ as we previously did to $\mathbf{Q}$ using the decomposition method. The probability matrix becomes

$$
\begin{aligned}
\mathbf{P}\left(t_{l-1}, t_{l+1}, \Psi\right) & =e^{\mathbf{Z}\left(t_{k}, t_{k+1}\right)} \\
& =\mathbf{E} \operatorname{diag}\left\{e^{\lambda_{1}}, \ldots, e^{\lambda_{k}}\right\} \mathbf{E}^{-1}
\end{aligned}
$$

We note that in this case the decomposition appears identical to the decomposition of $\mathbf{P}$ in terms of $\mathbf{Q}$, however in this case the eigenvalues $\lambda_{1}, \ldots, \lambda_{k}$ represent the eigenvalues of the $\mathbf{Z}$ matrix. We can proceed with a detailed breakdown of the likelihood analysis needed in order to obtain usable estimates for $\Psi$. The full model will be of use for panel data under a Markov assumption while incorporating B-Splines into the transition intensity matrix. 


\subsection{Penalized Likelihood Analysis}

The first step in likelihood analysis is to write down our likelihood function. At this time, we include the likelihood of a Markov structure as presented earlier, and a Weibull penalty term as a function of two parameters, $\alpha$ and $\gamma$. This likelihood function is given by

$$
\mathcal{L}(\underline{\Psi})=\prod_{l=1}^{m} \prod_{i=1}^{k} \prod_{j=1}^{k} p_{i j}\left(t_{l-1}, t_{l} ; \underline{\Psi}\right)^{n_{i j l}} e^{\left\{-\sum_{i \neq j} \lambda \int_{t_{0}}^{t_{l}}\left(\log \left(q\left(u ; \underline{\Psi}_{i j}\right)\right)-\log \left(\tilde{q}\left(u ; \alpha_{i j}, \gamma_{i j}\right)\right)\right)^{2} d u\right\}}
$$

where the first half of the equation is the likelihood of panel data under a Markov assumption and the second term is a penalty term. The penalty term is comprised of $q$, the transition intensity under the Markov assumption and $\tilde{q}$, which can be any intensity function thought to be appropriate. Here, we have chosen a Weibull hazard function with parameters $\alpha$ and $\gamma$. We define $\tilde{q}$ by

$$
\tilde{q}(t ; \alpha, \gamma)=\frac{\gamma}{\alpha}\left(\frac{t}{\alpha}\right)^{\gamma-1}
$$

We square the difference between these two functions and integrate, so that if the data deviates from a Weibull distribution, the penalty term increases, while if the data follows a Weibull distribution, the penalty term will be smaller. The parameter $\lambda$ allows the weight of the penalty term to fluctuate depending on the data set. When the data follows a Weibull distribution $\lambda$ is large and when it does not, $\lambda$ is small. The Weibull hazard function is parametrized by $\alpha$ and $\gamma$. However, we do not know what the true values of $\alpha$ and $\gamma$ are so we need to derive their maximum likelihood estimates, $\hat{\alpha}$ and $\hat{\gamma}$. We first work through the likelihood analysis of the panel data under a Markov assumption and under the assumption that $\alpha$ and $\gamma$ are known.

\subsection{Model-Based Penalties}

Model-based penalties are often a desirable penalty in situations where there is an apriori assumption of an underlying structure in the data. If the data closely follows the 
chosen model-based penalty, the penalty term will essentially force the model to fit the parametric model using maximum likelihood estimates. This leads to the smallest possible standard errors and bias by the properties of maximum likelihood estimates. Since the model-based penalty can be chosen as desired, the function can be any function from the space of continuous smooth functions. This gives a wide range of potential functions that can be considered. Even if a the data does not follow the chosen model-based penalty function very closely, an adjustment of the smoothness parameter $\lambda$ will provide flexibility to the model fit, incorporating more of the spline fit as $\lambda$ is lowered.

However, when the data deviates greatly from the assumed function used in the model-based penalty, it may be best to consider other options. Since derivatives penalties are standard practice and have proven to work well under many circumstances, they would be a good option to consider when looking for an alternative to the model based penalty. Depending on the data for each transition intensity, the modeler may choose to use different penalties for each transition intensity.

\subsubsection{Derivatives of the Likelihood Function}

To obtain the derivatives of the likelihood function, we will first obtain the derivations of the first component of the likelihood and then look at the derivatives of the penalty component. These derivatives will then be used to derive the score function and Fisher's Information matrix to be used in the Newton-Raphson method for estimating $\underline{\Psi}$.

We begin by taking the log of the original likelihood.

$\log \mathcal{L}(\underline{\Psi})=\sum_{l=1}^{m} \sum_{i=1}^{k} \sum_{j=1}^{k} n_{i j l} \log \left(p_{i j}\left(t_{l-1}, t_{l} ; \underline{\Psi}\right)\right)-\sum_{i \neq j} \lambda_{i j} \int_{t_{0}}^{t_{l}}\left(\log \left(q\left(t ; \underline{\Psi}_{i j}\right)\right)-\log \left(\tilde{q}\left(t ; \alpha_{i j}, \gamma_{i j}\right)\right)^{2} d t\right.$

Next, we take the partial derivatives of the first portion of the likelihood and obtain the following equation:

$$
\frac{\partial}{\partial \Psi_{i j c}} \sum_{l=1}^{m} \sum_{i=1}^{k} \sum_{j=1}^{k} n_{i j l} \log \left(p_{i j}\left(t_{l-1}, t_{l} ; \underline{\Psi}\right)\right)=\sum_{l=1}^{m} \sum_{i=1}^{k} \sum_{j=1}^{k} n_{i j l} \frac{\frac{\partial}{\partial \Psi_{i j c}} p_{i j}\left(t_{l-1}, t_{l} ; \underline{\Psi}\right)}{p_{i j}\left(t_{l-1}, t_{l} ; \underline{\Psi}\right)}(5.10)
$$


where $\Psi_{i j c}$ is the $c$ th spline coefficient in $\underline{\Psi}_{i j}$. Evidently, to calculate this quantity we must discuss how to obtain the first derivative of the probability function $p_{i j}\left(w_{l} ; \underline{\Psi}\right)$ with respect to the elements of $\underline{\Psi}$, the $\underline{\Psi}_{i j}$ 's. To do this, we first recall that we can write $\mathbf{P}$ as follows:

$$
\begin{aligned}
\mathbf{P}\left(t_{l-1}, t_{l} ; \underline{\Psi}\right) & =e^{\mathbf{Z}\left(t_{l-1}, t_{l} ; \underline{\Psi}\right)} \\
& =\sum_{s=0}^{\infty} \frac{\mathbf{Z}\left(t_{l-1}, t_{l} ; \underline{\Psi}\right)^{s}}{s !}
\end{aligned}
$$

Hence, the partial derivative of the probability matrix during the specific time panel is given by

$$
\begin{aligned}
\frac{\partial}{\partial \Psi_{i j c}} \mathbf{P}\left(t_{l-1}, t_{l} ; \Psi\right) & =\frac{\partial}{\partial \Psi_{p q}} \sum_{s=0}^{\infty} \frac{\mathbf{Z}\left(t_{l-1}, t_{l} ; \Psi\right)^{s}}{s !} \\
& =\sum_{s=0}^{\infty} \sum_{v=0}^{s-1} \mathbf{Z}\left(t_{l-1}, t_{l} ; \Psi\right)^{v} \frac{\partial \mathbf{Z}\left(t_{l-1}, t_{l} ; \Psi\right)}{\partial \Psi_{i j c}} \frac{\mathbf{Z}\left(t_{l-1}, t_{l} ; \Psi\right)^{s-v-1}}{s !}
\end{aligned}
$$

Here we can substitute the decomposed form $\mathbf{Z}\left(t_{l-1}, t_{l}\right)=\mathbf{E}_{l}(\underline{\Psi}) \boldsymbol{\Lambda}_{l}(\underline{\Psi}) \mathbf{E}_{l}(\underline{\Psi})^{-1}$ into the above equation. We also define

$$
\mathbf{G}_{l i j c}(\underline{\Psi})=\mathbf{E}_{l}(\underline{\Psi})^{-1} \frac{\partial \mathbf{Z}\left(t_{l-1}, t_{l} ; \underline{\Psi}\right)}{\partial \Psi_{i j c}} \mathbf{E}_{l}(\underline{\Psi})
$$

and we use it to simplify the computation for our derivative. Thus, the derivative becomes

$$
\begin{aligned}
\frac{\partial}{\partial \Psi_{i j c}} \mathbf{P}\left(t_{l}, t_{l+1} ; \Psi\right) & =\sum_{s=0}^{\infty} \sum_{v=0}^{s-1} \mathbf{E}_{l}(\underline{\Psi}) \boldsymbol{\Lambda}_{l}(\underline{\Psi})^{v} \mathbf{G}_{l p q}(\underline{\Psi}) \boldsymbol{\Lambda}_{l}(\underline{\Psi})^{s-v-1} \mathbf{E}_{l}(\underline{\Psi})^{-1} \frac{1}{s !} \\
& =\mathbf{E}_{l}(\underline{\Psi})\left(\sum_{s=0}^{\infty} \sum_{v=0}^{s-1} \boldsymbol{\Lambda}_{l}(\underline{\Psi})^{v} \mathbf{G}_{l i j c}(\underline{\Psi}) \boldsymbol{\Lambda}_{l}(\underline{\Psi})^{s-v-1} \frac{1}{s !}\right) \mathbf{E}_{l}(\underline{\Psi})^{-1} \\
& =\mathbf{E}_{l}(\underline{\Psi}) \mathbf{V}_{l i j c}(\underline{\Psi}) \mathbf{E}_{l}(\underline{\Psi})^{-1}
\end{aligned}
$$


where $\mathbf{V}_{l i j c}(\underline{\Psi})$ is a $k \times k$ matrix with $(p, q)$ elements defined as follows:

$$
\begin{aligned}
\mathbf{V}_{l i j c}^{(p q)}(\underline{\Psi}) & =\mathbf{G}_{l i j c}^{(p p)}(\underline{\Psi}) \sum_{s=0}^{\infty} \sum_{v=0}^{s-1} \lambda_{p}(\underline{\Psi})^{s-1} \frac{1}{s !} \\
& =\mathbf{G}_{l i j c}^{(p p)}(\underline{\Psi}) \sum_{s=0}^{\infty} \lambda_{p}(\underline{\Psi})^{s-1} \frac{1}{(s-1) !} \\
& =\mathbf{G}_{l i j c}^{(p p)}(\underline{\Psi}) e^{\lambda_{p}(\underline{\Psi})}
\end{aligned}
$$

when $p=q$ and

$$
\begin{aligned}
\mathbf{V}_{l i j c}^{(p q)}(\underline{\Psi}) & =\mathbf{G}_{l i j c}^{(p q)}(\underline{\Psi}) \sum_{s=0}^{\infty} \sum_{v=0}^{s-1} \lambda_{p}(\underline{\Psi})^{v} \lambda_{q}(\underline{\Psi})^{s-v-1} \frac{1}{s !} \\
& =\mathbf{G}_{l i j c}^{(p q)}(\underline{\Psi}) \sum_{s=0}^{\infty} \lambda_{q}(\underline{\Psi})^{s-1} \frac{1}{s !} \sum_{v=0}^{s-1}\left[\frac{\lambda_{p}(\underline{\Psi})}{\lambda_{q}(\underline{\Psi})}\right]^{v} \\
& =\mathbf{G}_{l i j c}^{(p q)}(\underline{\Psi}) \frac{1}{\lambda_{p}(\underline{\Psi})-\lambda_{q}(\underline{\Psi})} \sum_{s=0}^{\infty}\left[\lambda_{p}(\underline{\Psi})^{s}-\lambda_{q}\left(\underline{\Psi}^{s}\right] \frac{1}{s !}\right. \\
& =\mathbf{G}_{l i j c}^{(p q)}(\underline{\Psi}) \frac{e^{\lambda_{p}(\underline{\Psi})}-e^{\lambda_{q}(\underline{\Psi})}}{\lambda_{p}(\underline{\Psi})-\lambda_{q}(\underline{\Psi})}
\end{aligned}
$$

when $p \neq q$ where $\lambda_{p}(\underline{\Psi})$ is the $p$ th element of $\boldsymbol{\Lambda}_{l}(\underline{\Psi})$. This process is an extension of the work done by Kabfleisch and Lawless in 1985 to the spline case [7.

Now that we have all components necessary for the derivative of the first half of the likelihood function, we must calculate the derivative of the the penalty term. The computation for the derivative of the penalty term is much less complicated than that of the Markov assumption term and it can be simply derived as follows:

$$
\begin{aligned}
& \sum_{i \neq j} \frac{\partial}{\partial \Psi_{i j c}}\left(\lambda_{i j} \int_{t_{0}}^{t_{m}}\left(\log \left(q\left(t ; \underline{\Psi}_{i j}\right)\right)-\log \left(\tilde{q}\left(t ; \alpha_{i j}, \gamma_{i j}\right)\right)\right)^{2} d t\right) \\
= & \sum_{i \neq j} \frac{\partial}{\partial \Psi_{i j c}} \lambda_{i j} \int_{t_{0}}^{t_{m}}\left(\mathbf{B}(t)^{T} \underline{\Psi}_{i j}-\log \left(\gamma_{i j}\right)+\log \left(\alpha_{i j}\right)-\left(\gamma_{i j}-1\right) \log (t)+\left(\gamma_{i j}-1\right) \log \left(\alpha_{i j}\right)\right)^{2} d t \\
= & \lambda_{i j} \int_{t_{0}}^{t_{m}} 2 B_{c}(t)\left(\mathbf{B}(t)^{T} \underline{\Psi}_{i j}-\log \left(\gamma_{i j}\right)+\log \left(\alpha_{i j}\right)-\left(\gamma_{i j}-1\right) \log (t)+\left(\gamma_{i j}-1\right) \log \left(\alpha_{i j}\right)\right) d t .
\end{aligned}
$$


At this time we also calculate the second partial derivatives of the penalty term, as it will be used in the Fisher's Information calculation. Proceeding using the first derivative, we get:

$\frac{\partial^{2}}{\partial \Psi_{i j c} \partial \Psi_{i j d}}\left(\sum_{i \neq j} \lambda_{i j} \int_{t_{0}}^{t_{m}}\left(\log \left(q\left(t ; \Psi_{i j}\right)\right)-\log \left(\tilde{q}\left(t ; \alpha_{i j}, \gamma_{i j}\right)\right)^{2} d t\right)=2 \lambda_{i j} \int_{t_{0}}^{t_{m}} B_{c}(t) B_{d}(t) d t\right.$.

These derivatives can now be summed to obtain the full derivative of the likelihood function for the data. In order to estimate the parameter $\underline{\Psi}$, we use these derivatives to obtain the score function and Fisher Information matrix.

\subsubsection{The Score Function}

Since the score function is simply the first partial derivatives of the log-likelihood, we have already derived all of its components. The stacked $(d+k) \times 1$ vector elements of the score function $\mathbf{S}(\underline{\Psi})=\left\{S_{i j}(\underline{\Psi})\right\}$ are given by:

$$
\begin{aligned}
S_{i j}(\underline{\Psi})= & \frac{\partial}{\partial} \Psi_{i j} \log \mathcal{L}(\underline{\Psi}) \\
= & \left\{\sum_{l=1}^{m} \sum_{i=1}^{k} \sum_{j=1}^{k} n_{i j l} \frac{\frac{\partial}{\partial \Psi_{i j c}} p_{i j}\left(w_{l} ; \underline{\Psi}\right)}{p_{i j}\left(w_{l} ; \underline{\Psi}\right)}\right. \\
& \left.-\lambda_{i j} \int_{t_{0}}^{t_{m}} 2 B_{c}(t)\left(\mathbf{B}(t)^{T} \underline{\Psi}_{i j}-\log \left(\gamma_{i j}\right)+\log \left(\alpha_{i j}\right)-\left(\gamma_{i j}-1\right) \log (t)+\left(\gamma_{i j}-1\right) \log \left(\alpha_{i j}\right)\right) d t\right\} .
\end{aligned}
$$

\subsubsection{Fisher's Information Matrix}

Next, we derive Fisher's Information matrix which involves the second partial derivatives of the likelihood. As will be shown below, it is possible to obtain Fisher's Information without actually computing second derivatives for the Markov portion of the likelihood. This greatly decreases computation times, especially as the number of time panels being 
analyzed increases. In the context of the model, Fisher's Information is given by

$$
\begin{aligned}
E[ & \left.-\frac{\partial^{2}}{\partial \Psi_{p q s} \partial \Psi_{a b c}} \log \mathcal{L}(\underline{\Psi})\right] \\
& =\left[\sum_{l=1}^{m} \sum_{i, j=1}^{k} n_{i j l}\left\{\frac{\frac{\partial}{\partial \Psi_{p q s}} p_{i j}\left(w_{l} ; \underline{\Psi}\right) \frac{\partial}{\partial \Psi_{a b c}} p_{i j}\left(w_{l} ; \underline{\Psi}\right)}{p_{i j}^{2}\left(w_{l} ; \underline{\Psi}\right)}-\frac{\frac{\partial^{2}}{\partial \Psi_{p q s} \partial \Psi_{a b c}} p_{i j}\left(w_{l} ; \underline{\Psi}\right)}{p_{i j}\left(w_{l} ; \underline{\Psi}\right)}\right\}\right]+A
\end{aligned}
$$

where $A=2 \lambda \int_{t_{0}}^{t_{m}} B_{s}(t) B_{c}(t) d t$ if $a=p$ and $q=b$, zero otherwise. Note that we condition on $N_{i}\left(t_{l-1}\right)$, where $N_{i}\left(t_{l-1}\right)=\sum_{j=1}^{k} n_{i j l}$. This results in the counts per time period $n_{i j l}$ being multinomial with means $N_{i}\left(t_{l-1}\right) p_{i j}\left(w_{l} ; \underline{\Psi}\right)$, which simplifies the above equation as follows:

$$
\begin{aligned}
& =E\left[\sum_{l=1}^{m} \sum_{i, j=1}^{k} N_{i}\left(t_{l-1}\right)\left\{\frac{\frac{\partial}{\partial \Psi_{p q s}} p_{i j}\left(w_{l} ; \underline{\Psi}\right) \frac{\partial}{\partial \Psi_{a b c}} p_{i j}\left(w_{l} ; \underline{\Psi}\right)}{p_{i j}\left(w_{l} ; \underline{\Psi}\right)}-\frac{\partial^{2}}{\partial \Psi_{p q s} \partial \Psi_{a b c}} p_{i j}\left(w_{l} ; \underline{\Psi}\right)\right\}\right]+A \\
& =\sum_{l=1}^{m} \sum_{i=1}^{k} \sum_{j=1}^{k} \frac{E\left[N_{i}\left(t_{l-1}\right)\right]}{p_{i j}\left(w_{l} ; \underline{\Psi}\right)} \frac{\partial}{\partial \Psi_{p q s}} p_{i j}\left(w_{l} ; \underline{\Psi}\right) \frac{\partial}{\partial \Psi_{a b c}} p_{i j}\left(w_{l} ; \underline{\Psi}\right)+A .
\end{aligned}
$$

The second derivative term has disappeared from the last equation as we have constructed $\mathbf{P}$ such that the sum of its rows is 1 . Hence, when the summation $\sum_{j=1}^{k} \frac{\partial^{2}}{\partial \Psi_{p q s} \partial \Psi_{a b c}} p_{i j}\left(w_{l} ; \underline{\Psi}\right)$ is applied, $p_{i j}\left(w_{l} ; \underline{\Psi}\right)=1$ and thus its derivative is 0 . This makes it possible to obtain Fisher's Information without actually computing second derivatives of $\mathbf{P}$. Finally, to get a reasonable estimate for Fisher's Information, we simply replace the expected value of $N_{i}\left(t_{l-1}\right)$ with its observed value

$$
\mathbf{M}_{p q a b}(\underline{\Psi})=\left\{\sum_{l=1}^{m} \sum_{i=1}^{k} \sum_{j=1}^{k} \frac{N_{i}\left(t_{l-1}\right)}{p_{i j}\left(w_{l} ; \underline{\Psi}\right)} \frac{\partial}{\partial \underline{\Psi}_{p q s}} p_{i j}\left(w_{l} ; \underline{\Psi}\right) \frac{\partial}{\partial \underline{\Psi}_{a b c}} p_{i j}\left(w_{l} ; \underline{\Psi}\right)+A\right\}
$$

where $\mathbf{M}_{p q a b}(\underline{\Psi})=\frac{\partial^{2}}{\partial \underline{\Psi}_{p q} \partial \underline{\Psi}_{a b}^{T}} \log \mathcal{L}(\underline{\Psi})$. This gives us the observed Fisher's Information, with the pairs $p, q$ and $a, b$ representing the element of the $\underline{\boldsymbol{\Psi}}$ vector corresponding to a specific transition. The full Fisher's information is then obtained from all the $\mathbf{M}_{p q a b}(\underline{\Psi})$ 's where $\mathbf{M}_{a b p q}(\underline{\Psi})=\mathbf{M}_{p q a b}(\underline{\Psi})$. We are hence able to obtain an estimate for $\underline{\Psi}$ using the 
Fisher's scoring method as follows:

$$
\underline{\Psi}^{(r+1)}=\underline{\Psi}^{(r)}+\mathbf{M}(\underline{\Psi})^{-1} \mathbf{S}(\underline{\Psi})
$$

until convergence. Finally, we need to derive the estimates for $\alpha$ and $\gamma$ of the Weibull penalty before we can truly calculate estimates for $\underline{\Psi}$. These estimates are obtained similarly to the estimate for $\underline{\Psi}$ and are calculated prior to the likelihood analysis done here.

\subsubsection{The Weibull Penalty: Maximum Likelihood Parameter Estimates}

The hazard function or intensity of a Weibull distribution is given by

$$
h(t ; \alpha, \gamma)=\frac{\gamma}{\alpha}\left(\frac{t}{\alpha}\right)^{\gamma-1}
$$

and the probability density function (pdf) is given by

$$
f(t ; \gamma, \alpha)=\frac{\gamma}{\alpha}\left(\frac{t}{\alpha}\right)^{\gamma-1} e^{-\left(\frac{t}{\alpha}\right)^{\gamma}}
$$

To get the maximum likelihood estimates for $\alpha$ and $\gamma$ we follow the same process as we did for the spline transition intensities above. However, now the intensity is the Weibull hazard function so we have:

$$
\tilde{q}\left(t, \alpha_{i j}, \gamma_{i j}\right)=\frac{\gamma_{i j}}{\alpha_{i j}}\left(\frac{t}{\alpha_{i j}}\right)^{\gamma_{i j}-1}
$$

Since the intensity is now being modeled as a Weibull hazard function, we not have no penalty term as the hazard function becomes the sole component in the equation for $q_{i j}$.

Next, we repeat the likelihood analysis in order to get estimates for $\alpha_{i j}$ and $\gamma_{i j}$ using the Newton Raphson method with the transition intensity model. We can first write

$$
\tilde{\mathbf{Z}}\left(t_{l-1}, t_{l} ; \alpha_{i j}, \gamma_{i j}\right)=\int_{t_{l-1}}^{t_{l}} \frac{\gamma_{i j}}{\alpha_{i j}}\left(\frac{u}{\alpha_{i j}}\right)^{\gamma_{i j}-1} d u
$$


and we obtain $\tilde{\mathbf{P}}\left(t_{l-1}, t_{l}\right)$ and its derivatives in terms of $\tilde{\mathbf{Z}}\left(t_{l-1}, t_{l} ; \alpha_{i j}, \gamma_{i j}\right), l=1, \ldots, m$. Now that $\tilde{\mathbf{Z}}$ is in terms of $\alpha_{i j}$ and $\gamma_{i j}$, we can find derivatives of $\mathbf{P}\left(\tilde{t_{l-1}}, t_{l}\right)$ and thus obtain the score function and Fisher's Information of this system of equations. The score function for a Weibull transition intensity is given by

$$
\tilde{S}\left(\alpha_{i j}\right)=\frac{\partial}{\partial \alpha_{i j}} \log \mathcal{L}(\underline{\alpha}, \underline{\gamma})
$$

and

$$
\tilde{S}\left(\gamma_{i j}\right)=\frac{\partial}{\partial \gamma_{i j}} \log \mathcal{L}(\underline{\alpha}, \underline{\gamma})
$$

Finally, the Fisher Information matrix is given by

$$
\left[\begin{array}{ll}
E\left[-\frac{\partial^{2}}{\partial \alpha_{i j} \partial \alpha_{i j}} \log \mathcal{L}(\underline{\alpha}, \underline{\gamma})\right] & E\left[-\frac{\partial^{2}}{\partial \alpha_{i j} \partial \gamma_{i j}} \log \mathcal{L}(\underline{\alpha}, \underline{\gamma})\right] \\
E\left[-\frac{\partial^{2}}{\partial \alpha_{i j} \partial \gamma_{i j}} \log \mathcal{L}(\underline{\alpha}, \underline{\gamma})\right] & E\left[-\frac{\partial^{2}}{\partial \gamma_{i j} \partial \gamma_{i j}} \log \mathcal{L}(\underline{\alpha}, \underline{\gamma})\right]
\end{array}\right]
$$

where $\underline{\alpha}$ and $\underline{\gamma}$ are vectors of all Weibull parameters for all transitions. Estimates for $\alpha$ and $\gamma$ are found by using Fisher's scoring with the equations above for all $i$ to $j$ transtions until convergence. These values now replace the penalty parameters in the Weibull hazard portion of the likelihood function.

\subsection{Estimates of Derived Quantities}

Though we are able to find the score and Fisher Information for the estimated parameters $\underline{\underline{\Psi}}$, it is often of interest to find information about a function of the parameter, say $f(\underline{\Psi})$. In the case of the above discussed model, we are interested in the number of transitions from one state to another at a specific time. This corresponds to the $q(t)$ explained in Chapter 3. Recall that

$$
q(t)=e^{\underline{\mathbf{B}}(t)^{T}} \underline{\Psi}_{i j}
$$


which is clearly a function of the parameter of interest. Let $f\left(\underline{\Psi}_{i j}\right)=q(t)$ and thus we can estimate $f\left(\underline{\Psi}_{i j}\right)$ using $f\left(\underline{\underline{\Psi}}_{i j}\right)=\hat{q}(t)$ where $\hat{q}(t)=e^{\underline{\mathbf{B}}(t)^{T} \underline{\underline{\Psi}}_{i j}}$.

Using this estimated function we can gather many quantities useful for analysis, such as the estimated variance, which can then lead us to other important information such as standard errors and confidence intervals. In order to find the estimated variance, we use the Delta Method. This gives

$$
\widehat{\operatorname{Var}}(f(\underline{\hat{\Psi}})) \approx \nabla f(\underline{\hat{\Psi}})^{T} M(\underline{\hat{\Psi}})^{-1} \nabla f(\underline{\hat{\Psi}})
$$

as the estimated variance of the $q(t)$. We note that $\nabla f(\underline{\hat{\Psi}})$ is given by

$$
\nabla q(t) \approx\left\{B_{v}(t) e^{\underline{\mathbf{B}}^{T}(t)} \underline{\Psi}_{i j}\right\}_{g \times 1}
$$

where $g$ is the number of spline coefficients. This is the process by which estimated quantities are obtained from the simulation results, which will be presented in the following chapter. 


\section{Simulation Study}

In order to evaluate the statistical properties of penalized spline model estimators, we ran multiple simulations through the model with varying factors. The idea behind these simulations was to understand how the influence of the penalty parameter $\lambda$ affects the results for both functions that are of the same functional form as the penalty (Weibull in this case) and those that are not.

Under the construction of the model, it is believed that when the underlying structure of the data comes from a Weibull intensity, the penalty parameter $\lambda$ will be large in order to force the penalty term to account for most of the model. The spline model component only plays a small role in this case, which decreases as $\lambda \rightarrow \infty$. Essentially, this case is equivalent to fitting a Weibull intensity using maximum likelihood estimates. Conversely, when the underlying structure of the data does not follow a Weibull intensity, $\lambda$ will be small in order for the spline model to be the primary component of the model. Ideally, an optimization method would be used to predict the most appropriate $\lambda$ for each data set. In our simulation, the $\lambda$ 's were fixed and chosen based on results obtained through numerous repetitions of model fitting that produced desirable results and minimal failures. This was done as the current code for fitting is not numerically efficient. In future studies, a data driven $\lambda$ value is essential to provide key information and more realistic results. These potential options will be outlined in the discussion.

It was also of interest to determine if the model could fit both types of data well, proving its robustness in a variety of scenarios. However, with this in mind, we realize that fitting a function that is completely non-Weibull under the assumption that it is Weibull will not produce ideal results and a usual penalized spline model would be a better choice in such cases. The purpose of the penalty here is to showcase its effectiveness when apriori knowledge of an underlying functional structure is a reasonable assumption. 


\subsection{Methodology}

The varying factors of the simulation study included the number of participants in the generated data, $n$, and the number of follow-up periods or time panels per individual, $l$. Three values of each were chosen to obtain an 3 by 3 factorial study that demonstrates the effectiveness and restrictions of the model. The levels chosen for $n$ were 150, 250 and 500. These are based upon potential sufficient, good and very good numbers of participants in a clinical trial. The values chosen for the number of panels $l$ were 10 , 25 and 50. Again, these represent acceptable, good and very good numbers for the number of follow up times in a trial. Pre-simulation results suggested the case with 500 participants and 50 follow-up times was the best case scenario, and the cases with 150 particpants and 10 follow-up times was minimally effective. A large number of equally spaced knots, 20, was used to ensure the penalty term could act on the fit of the function. The time period $[0.5,5]$ was divided equally depending on the number of time periods needed, as at time 0 computational issue swere encountered. All simulations were run in $R$ [16]. The full scope of results will be evident through the analysis of all 9 possible cases.

Two functions were used to generate data. One of the functions was very nonWeibull, while the other was generated directly from a Weibull intensity function. The non-Weibull function was a positive quadratic with the following form:

$$
q_{12}(x)=(x-3)^{2}+1
$$

whereas the Weibull function took on the following parametrization of the Weibull intensity function:

$$
q_{21}(x)=(0.8 / 0.2)(x / 0.2)^{(0.8-1)}
$$

That is, $\alpha=0.2$ and $\gamma=0.8$. These parameter values were chosen such that the Weibull intensity could not fi the quadratic function being used. The data used in the 
simulation was generated from a two state Markov process with these two functions as intensity functions, while the model was then fit using the procedure outlined in the previous chapter. The goal of the simulation was also to demonstrate how the model reacts to Weibull and non-Weibull functions. As such, a much larger $\lambda$ value was used for the Weibull function than the ones used for the non-Weibull function. The full breakdown of $\lambda$ values used and the subsequent failure rates due to convergence taking longer than 50 iterations is shown in Table 1.

\begin{tabular}{cccccc}
$n$ & $l$ & $\lambda_{\text {Non-Weibull }}$ & $\lambda_{\text {Weibull }}$ & Successful Runs & Failure Rate \\
\hline 150 & 10 & 9 & 10000 & $453 / 500$ & $9.4 \%$ \\
& 25 & 6 & 10000 & $489 / 500$ & $2.2 \%$ \\
& 50 & 4 & 10000 & $500 / 500$ & $0.0 \%$ \\
\hline 250 & 10 & 15 & 10000 & $478 / 500$ & $4.4 \%$ \\
& 25 & 10 & 10000 & $495 / 500$ & $1.0 \%$ \\
& 50 & 8 & 10000 & $479 / 500$ & $4.2 \%$ \\
\hline 500 & 10 & 27 & 10000 & $480 / 500$ & $4.0 \%$ \\
& 25 & 11 & 10000 & $496 / 500$ & $0.8 \%$
\end{tabular}

Table 1: Simulation Case Breakdown

All subsequent graphs generated from these cases used fit obtained from the number of successful runs, even if this number was less than 500. For all graphs, the time interval $[0.5,5]$ was divided into 1000 time points. The graphs for fit show the true function the data was generated from in blue and the resulting model fit in red at each of these 1000 time points. The bias was calculated by taking the average $q$ value over the total number of simulation runs and substracting the true value of $q$ at each of the 1000 time points. For coverage probabilities, confidence intervals were constructed for the $q$ value at each 
of the 1000 time points for each run. Then the counts of the number of times the true value of $q$ fell within the interval were aggregated. These numbers were divided by the total number of successful runs to get the resulting probability.

A couple of intermediate cases were also simulated to provide further understanding of the potential of the model. These consisted of a single run of the $n=500$ and $l=50$ case in which the quadratic function used a divided difference penalty term instead of the proposed model-based penalty term. The parameter $\lambda$ was held consistent at a value of 11. Once again, all graphs were produced using 1000 time points.

Then, a single run using a log-normal probability distribution function as $q_{12}(t)$ was used to evaluate the model's effectiveness on an intensity that was not Weibull, but could still be reasonably approximated using the Weibull penalty. The log-normal intensity function had the following parametrization:

$$
q_{12}(x)=e^{-\frac{\left((\log ((x-\theta) / m))^{2} /\left(2 \sigma^{2}\right)\right)}{(x-\theta) \sigma \sqrt{2 \pi}}}
$$

with $\theta=0, \sigma=1.5$ and $m=2$. It was determined via repeated fitting that a Weibull hazard function with $\alpha=0.7$ and $\gamma=0.2$ could approximate the log-normal function reasonably well. The penalty parameter $\lambda$ was set to 40 to ensure that the penalty term had influence on the model fit. Once again, $n$ and $l$ were kept at 500 and 50 respectively. The resulting graphs were created using 1000 time points.

The results of each case will be analyzed in the discussion. 


\section{Discussion}

The simulation results provided great insight into the functionality of the earlier proposed model. For the most part, the model behaved as anticipated and the fit varied according to the parameters specified, $n$ the number of individuals and $l$ the number of time panels. The comparison of a very non-Weibull function to a truly Weibull function demonstrated the functional penalty term's effectiveness while also shedding light on cases where using a simpler penalty term may be more effective. A single simulation run using a divided difference penalty for the non-Weibull function was performed. Furthermore, we compared the bias of the fits as well as coverage probabilities for all cases.

In order to compute coverage probabilities, we calculated the variance of the expected number of transitions per time period (i.e. $\mathbf{q}(t)$ ) using the Delta Method and then found a $95 \%$ point-wise confidence intervals $(\alpha=0.05)$ for $\mathbf{q}(t)$ at each time point for each run in the simulation. There were 500 runs per simulation case. Then, we tallied how many times the true value of $\mathbf{q}(t)$ fell within the confidence interval at each time. The coverage probability at each time point is thus given by the number of $\mathbf{q}(t)$ 's within the confidence interval divided by the total number of successful runs. For a very good fit, we expected coverage probabilities to be around the $95 \%$ nominal level.

Lastly, a single simulation case of a more Weibull, but not truly Weibull function was run in order to understand a potential middle case, where the model based penalty is able to fit a non-Weibull function well. All of these results gave way to new ideas and options for fitting different data in the future. Best approaches for various cases will be discussed as we break the results down according to the true intensity functions from which the data was generated. This information will allow us to provide recommendations for future work. We begin now by investigating the case that should always provide the best results: data from an underlying function that matches the model-based penalty. 


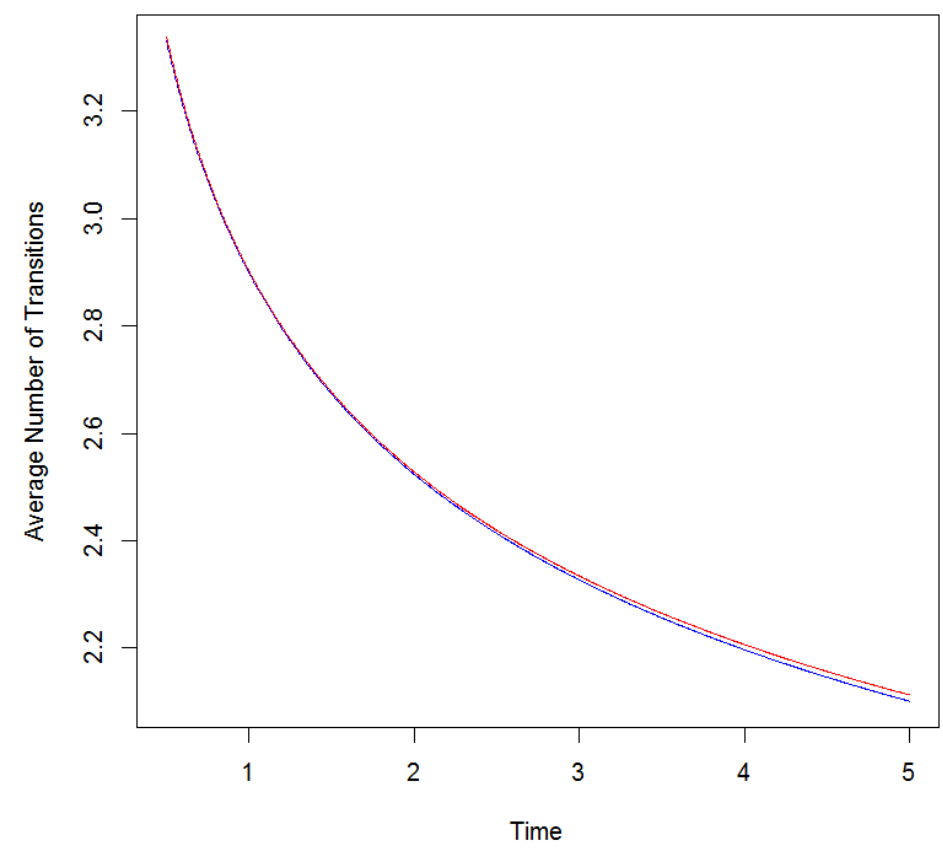

Figure 4: Weibull fit in the case with 150 individuals and 10 time panels

\subsection{Fitting a Weibull Function}

One of the functions chosen for the simulations $q_{12}(t)$ was a Weibull hazard function with parameters $\alpha=0.2$ and $\gamma=0.8$. The purpose of the model-based penalty is to fit such functions best, as the penalty term with a large $\lambda$ fits the data essentially using maximum likelihood estimates. Upon examination of several simulation cases, it is clear that even with a small sample the fit is very good. For instance, consider the comparison of the true underlying Weibull hazard function to the fit obtained through the use of a penalized spline and model-based penalty with $n=150$ and $l=10$ (shown in Figure 4).

We see that the red line representing the fit obtained through averaging the results of simulations for this case falls almost exactly along the blue line, which represents the true underlying function. As expected, if we investigate the fit obtained from the case with the highest number of individuals and panels in Figure 5, we see almost a perfect fit. When comparing the Weibull fits for the other seven cases, no visibly noticeable changes are found when decreasing the number of individuals or the number of panels. 


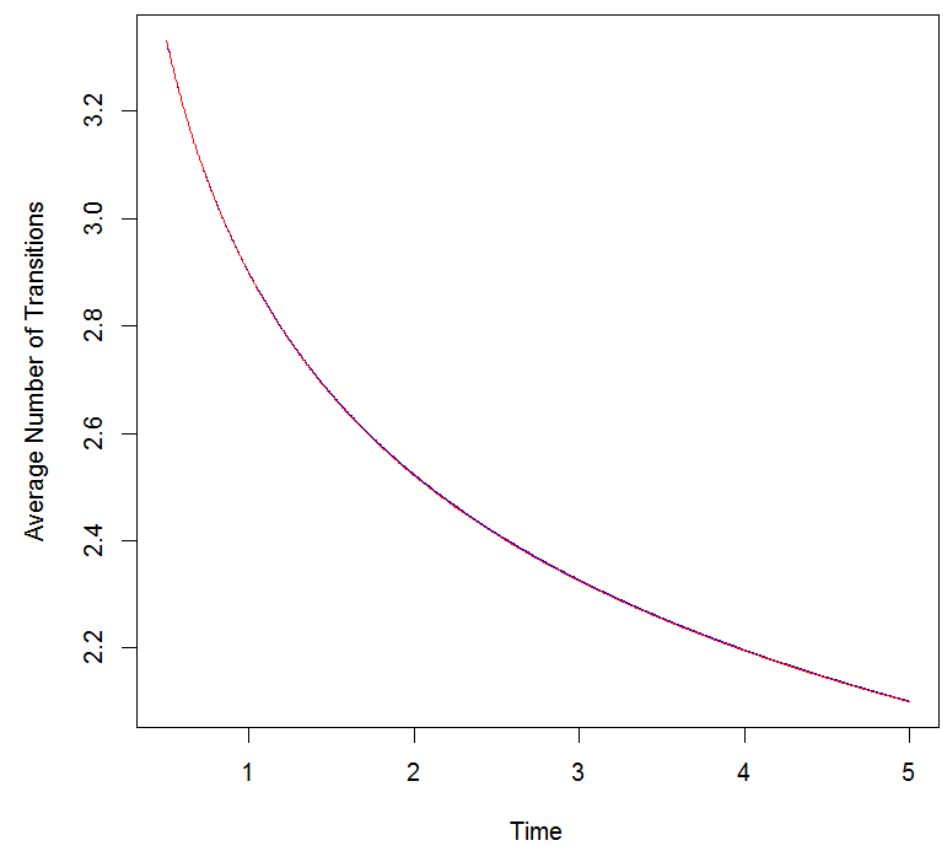

Figure 5: Weibull fit in the case with 500 individuals and 50 time panels

In all cases, the model based penalty approach performs very well.

While the fits seem similar visually, it is important to consider bias as well. Figures 6 and 7 demonstrate the bias between the true and the expected functions. We see that the bias is noticeably larger for the function with less individuals and time panels. The bias tends to increase with time. This is because the function does not fit as well towards the end of the time period (as seen in Figure 4). However, for the function with more individuals and time panels, the bias is much smaller and fairly constant over time (Figure 7).

One result that is interesting to note is that an increase in the number of individuals reduces bias. For instance, consider Figures 8 and 9. In Figure 8, the number of individuals has increased from 150 to 250 and there is already a noticeable reduction in bias. This reduction is furthered by the addition of more individuals (up to 500), yet the number of time panels used in the simulations did not change. The scale on these Figures varies in order to better highlight the changes in bias. On the original scale, the 


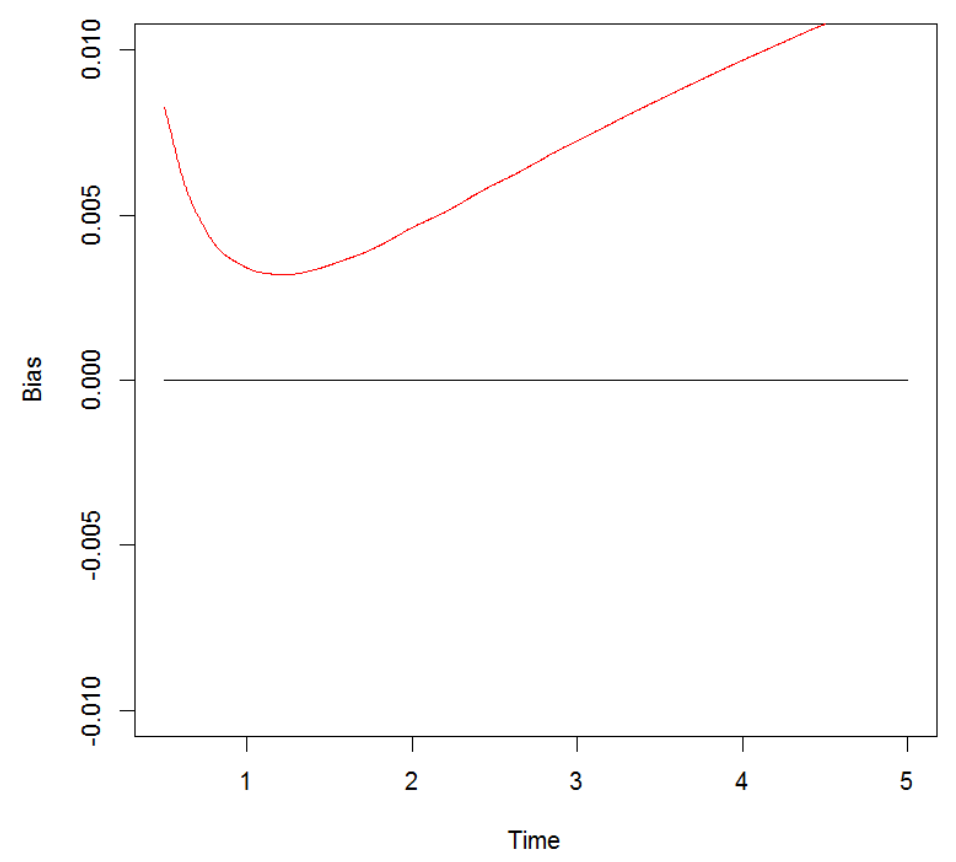

Figure 6: Weibull bias in the case with 150 individuals and 10 time panels

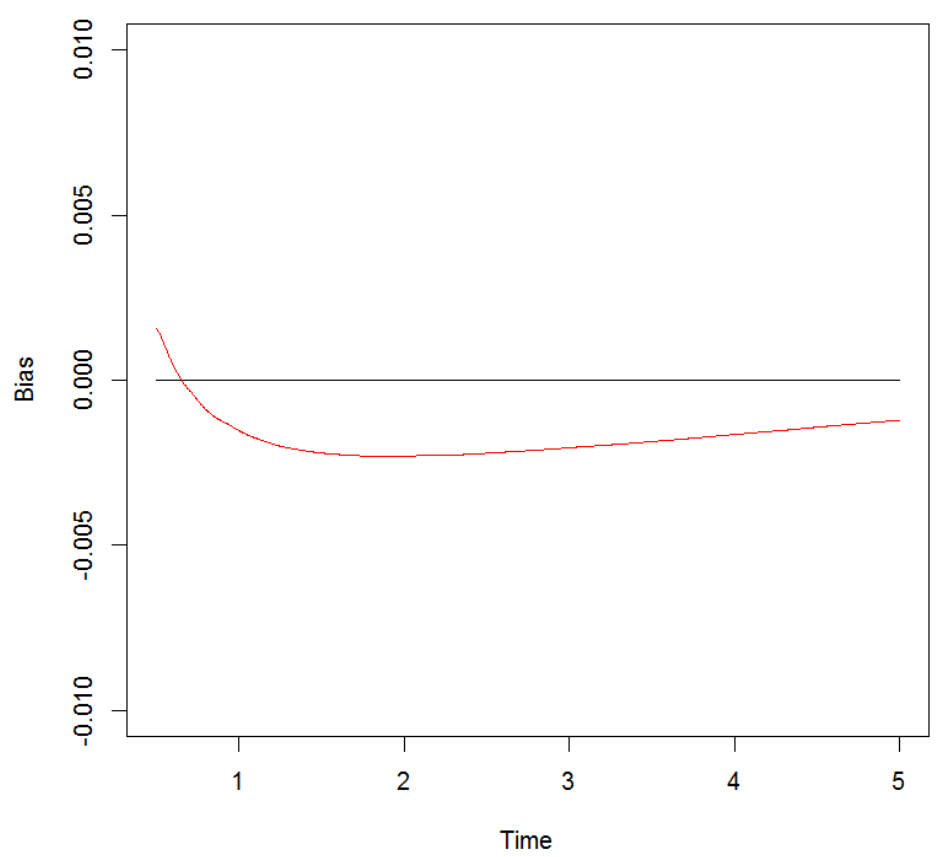

Figure 7: Weibull bias in the case with 500 individuals and 50 time panels 


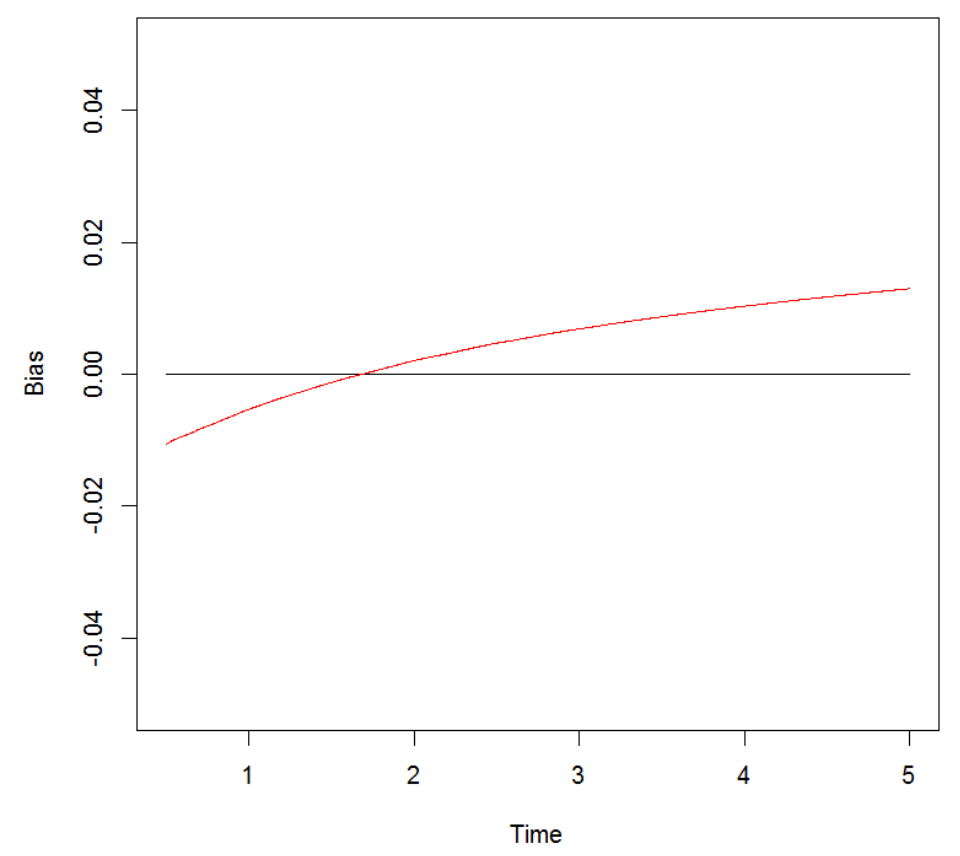

Figure 8: Weibull bias in the case with 250 individuals and 10 time panels

bias plots for any of the simulations with 500 time panels appear to be centered at zero, as seen in Figure 10. 


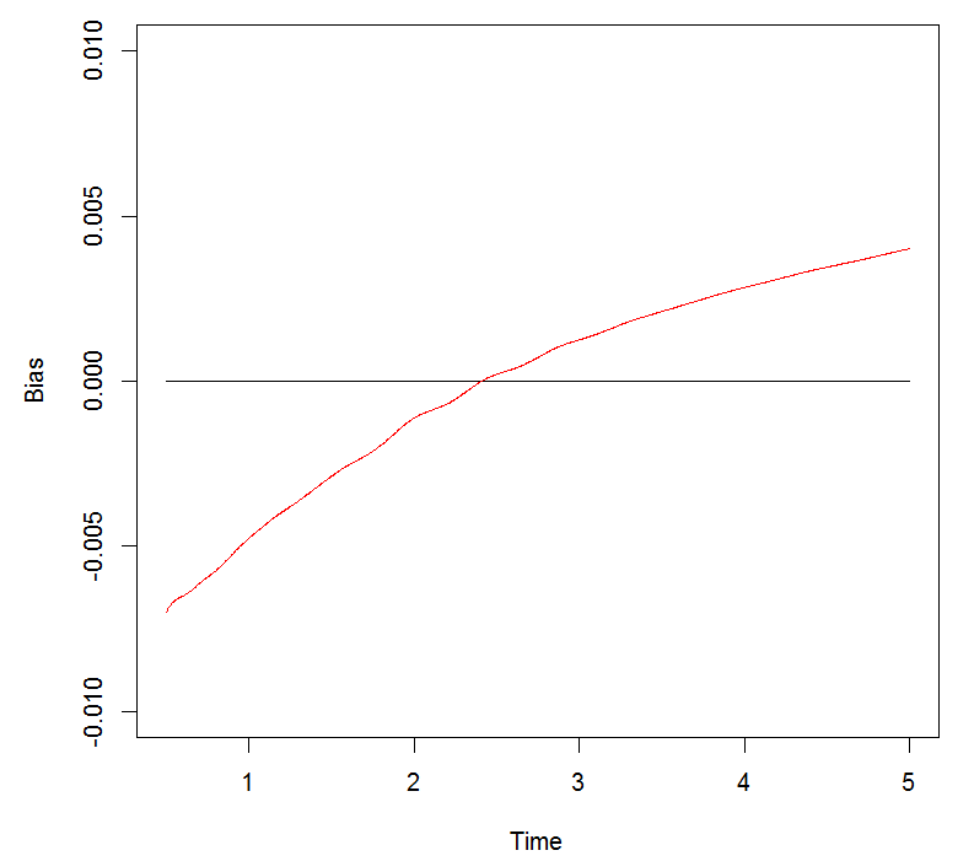

Figure 9: Weibull bias in the case with 500 individuals and 10 time panels

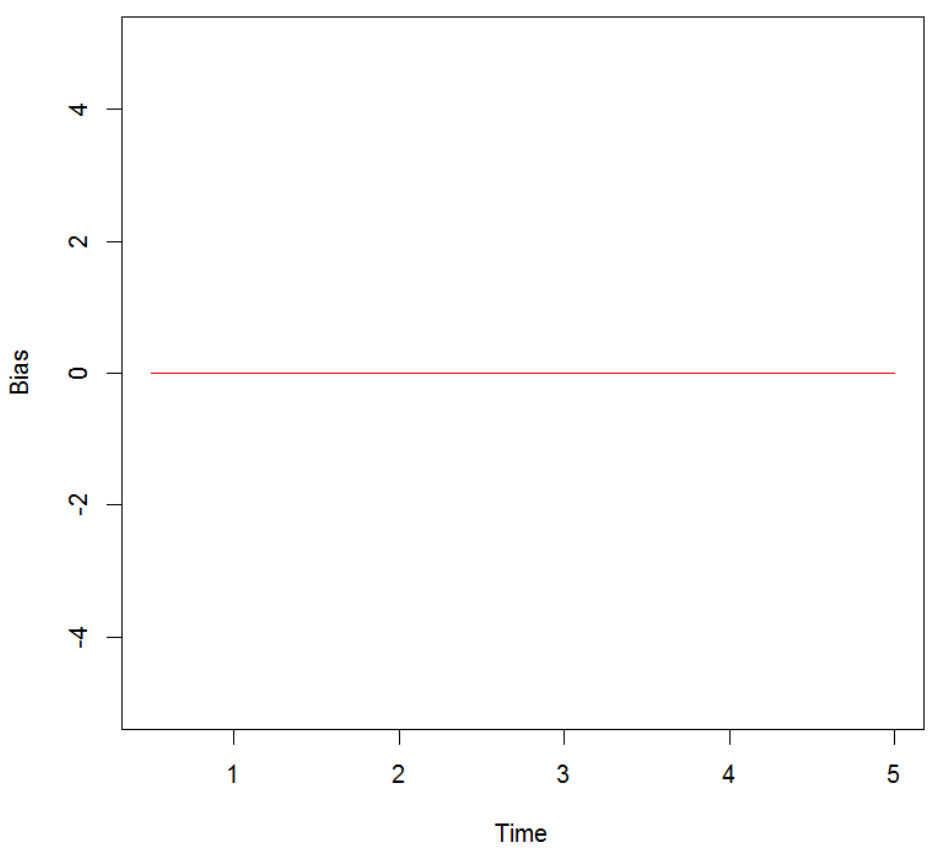

Figure 10: Weibull bias in the case with 500 individuals and 10 time panels. This graph is kept at the same scale as the graphs with fewer individuals. 
Furthermore, when the number of time panels is increased and the number of individuals is held constant, we can see that the bias reduces. In fact, increasing the number of time panels provides more information per individual, which in turn reduces the bias more than simply following additional individuals. Figures 11 and 12 demonstrate this difference, as the bias becomes very small following the increase of time panels from 25 to 50 .

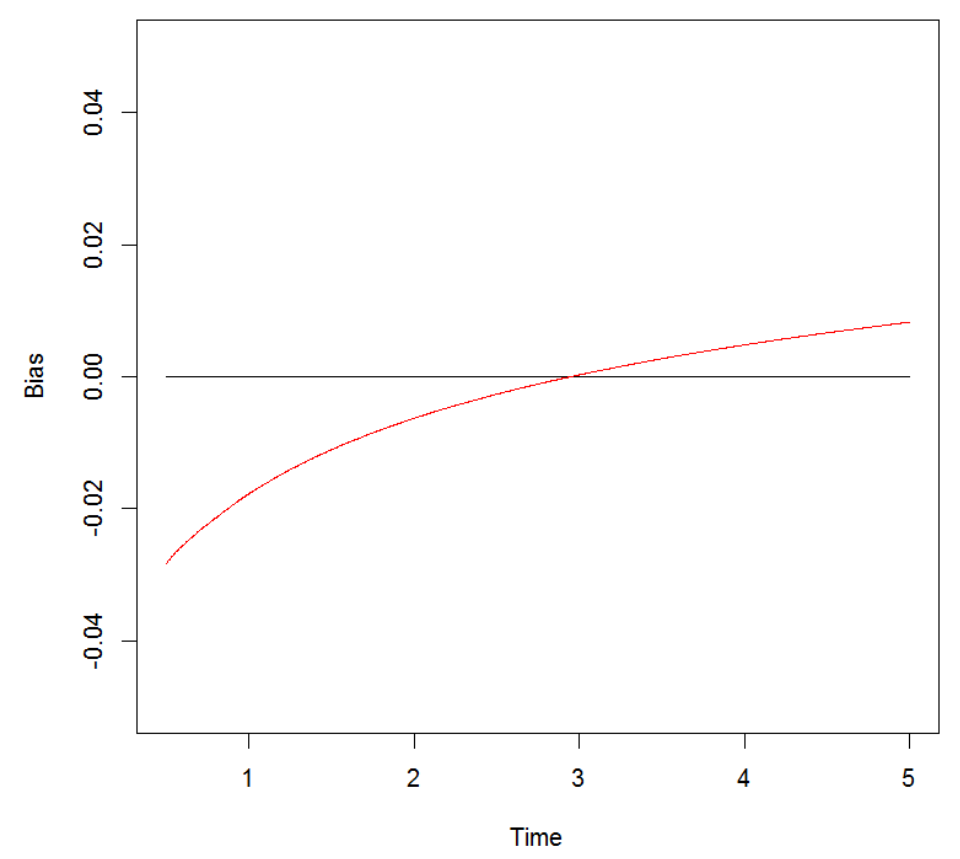

Figure 11: Weibull bias in the case with 250 individuals and 25 time panels

The last quantity to consider is coverage probability. Seeing as the model performs extremely well with an underlying Weibull intensity function, we expect these probabilities to be very high. However, since $\lambda$ is large, we must compute these using the asymptotic variance of the Weibull hazard function. This process is described in the following section. 


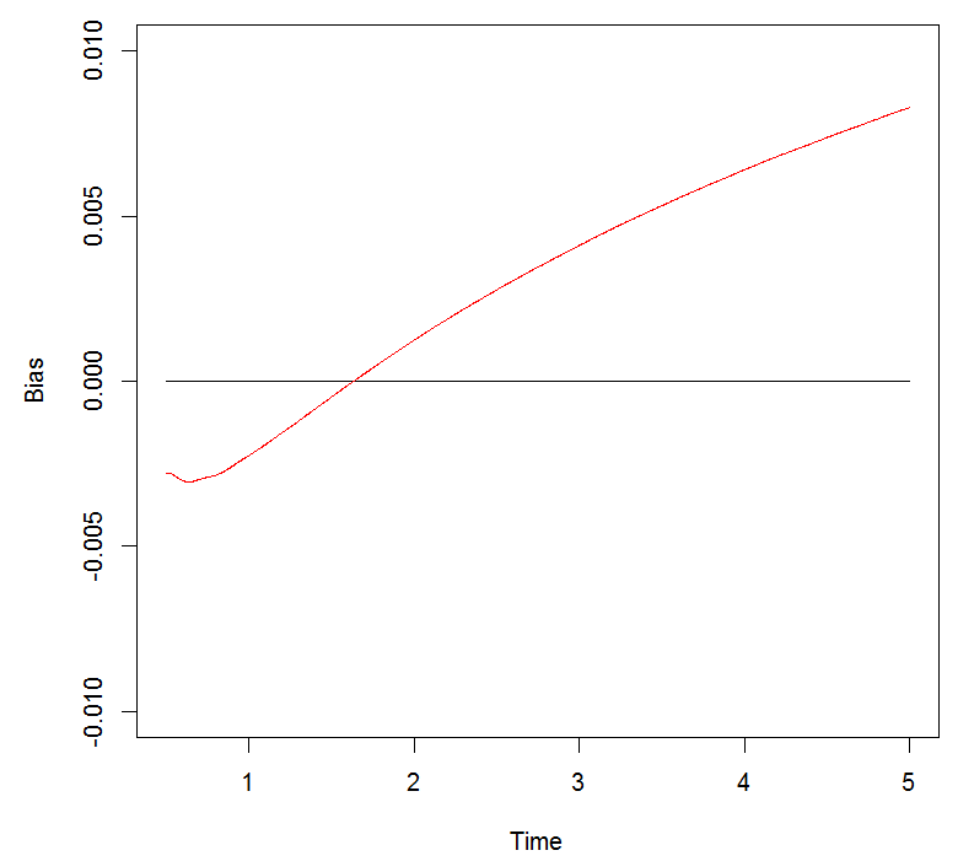

Figure 12: Weibull bias in the case with 250 individuals and 50 time panels

\subsubsection{The Influence of $\lambda$ on Derived Quantities}

It is important to realize that when $\lambda$ becomes very large, such as in all simulation cases for the Weibull intensity function, the Fisher Information portion of the M matrix no longer has any true influence on the M matrix. Essentially, the matrix is comprised of the second portion only. That is, the M matrix becomes:

$$
M \approx 2 \lambda\left\{\int_{t_{0}}^{t_{l}} B_{j}(t) B_{i}(t) d t\right\}
$$

Furthermore, any assumptions made due to normality are no longer valid, since the data are not being used to calculate standard deviations or variances. Thus, in order to determine the true variance covariance matrix, a different approach is needed.

Recall that when $\lambda$ is very large, the model essentially fits a Weibull intensity function using maximum likelihood estimates as only the penalty term is being used. Using this knowledge, we propose that in the case that $\lambda$ is sufficiently large, that the variance of 
the Weibull hazard function is used in all calculations involving derived quantities. In order to obtain this quantity, we must use the Delta Method on each parameter, $\alpha$ and $\gamma$. We begin by writing the transition intensity as the the Weibull hazard.

$$
q_{i j}\left(t ; \alpha_{i j}, \gamma_{i j}\right)=\frac{\gamma_{i j}}{\alpha_{i j}}\left(\frac{t}{\alpha_{i j}}\right)^{\left(\gamma_{i j}-1\right)}
$$

Then we follow the same procedure as described for the spline model previously to obtain estimates for $\alpha_{i j}$ and $\gamma_{i j}$. That is, we set

$$
\begin{aligned}
\mathbf{Z}\left(t_{l-1}, t_{l} ; \alpha_{i j}, \gamma_{i j}\right) & =\int_{t_{l-1}}^{t_{l}} q_{i j}\left(t ; \alpha_{i j}, \gamma_{i j}\right) d t \\
& =\int_{t_{l-1}}^{t_{l}} \frac{\gamma_{i j}}{\alpha_{i j}}\left(\frac{t}{\alpha_{i j}}\right)^{\left(\gamma_{i j}-1\right)} d t
\end{aligned}
$$

We then proceed using the decomposition method to find the score and Fisher's information as before, but without the addition of a penalty term. This will give us $\mathbf{S}\left(\alpha_{i j}, \gamma_{i j}\right)$ and $\mathbf{M}\left(\alpha_{i j}, \gamma_{i j}\right)$. We obtain estimates for each parameter using an iterative method until convergence, just as we did for earlier parameter estimates.

To use the Delta Method, we require the derivatives of $q_{i j}\left(t ; \hat{\alpha}_{i j}, \hat{\gamma}_{i j}\right)$ with respect to $\hat{\alpha}_{i j}$ and $\hat{\gamma}_{i j}$. We get

$$
\begin{aligned}
& \frac{\partial q_{i j}\left(t ; \hat{\alpha}_{i j}, \hat{\gamma}_{i j}\right)}{\partial \hat{\alpha}_{i j}}=\frac{-\hat{\gamma}_{i j}^{2}}{\hat{\alpha}_{i j}^{2}}\left(\frac{t}{\hat{\alpha}_{i j}}\right)^{\left(\hat{\gamma}_{i j}-1\right)} \\
& \frac{\partial q_{i j}\left(t ; \hat{\alpha}_{i j}, \hat{\gamma}_{i j}\right)}{\partial \hat{\gamma}_{i j}}=\left(\log \left(\frac{t}{\hat{\alpha}_{i j}}\right)+\frac{1}{\hat{\gamma}_{i j}}\right) \frac{\gamma_{i j}}{\alpha_{i j}}\left(\frac{t}{\alpha_{i j}}\right)^{\left(\gamma_{i j}-1\right)}
\end{aligned}
$$

Then we have

$$
\nabla q_{i j}(t ; \hat{\alpha}, \hat{\gamma})=\left[\begin{array}{l}
\frac{\partial q_{i j}\left(t ; \hat{\alpha}_{i j}, \hat{\gamma}_{i j}\right)}{\partial \hat{\alpha}_{i j}} \\
\frac{\partial q_{i j}\left(t ; \hat{\alpha}_{i j}, \hat{\gamma}_{i j}\right)}{\partial \hat{\gamma}_{i j}}
\end{array}\right] .
$$

By the Delta Method, we can conclude that the variance of $q_{i j}\left(t ; \hat{\alpha}_{i j}, \hat{\gamma}_{i j}\right)$ at $t$ is given 
by

$$
\operatorname{Var}\left(q_{i j}\left(t ; \hat{\alpha}_{i j}, \hat{\gamma}_{i j}\right)\right)=\nabla q_{i j}\left(t ; \hat{\alpha}_{i j}, \hat{\gamma}_{i j}\right)^{T} \mathbf{M}\left(\hat{\alpha}_{i j}, \hat{\gamma}_{i j}\right)^{-1} \nabla q_{i j}\left(t ; \hat{\alpha}_{i j}, \hat{\gamma}_{i j}\right)
$$

Using this quantity we can compute confidence intervals for uses such as coverage probabilities. This is the method that was used to produce the coverage probabilities for the simulation cases of the Weibull function.

\subsubsection{Coverage Probabilities for the Weibull Function}

Using the process described above, we were able to compute coverage probabilities for the Weibull intensity function in all cases. The coverage probabilities were very high and consistently around 0.95 for all cases as is expected with enough data points. As shown in figures 13 and 14 , in both the lowest level $(n=150$ and $l=10)$ and the highest ( $n=500$ and $l=50$ ) cases, the coverage probabilities look reasonable. This is expected as both the fits and biases for these cases were quite good as well since the model-based penalty led to essentially fitting maximum likelihood estimates in this case. 


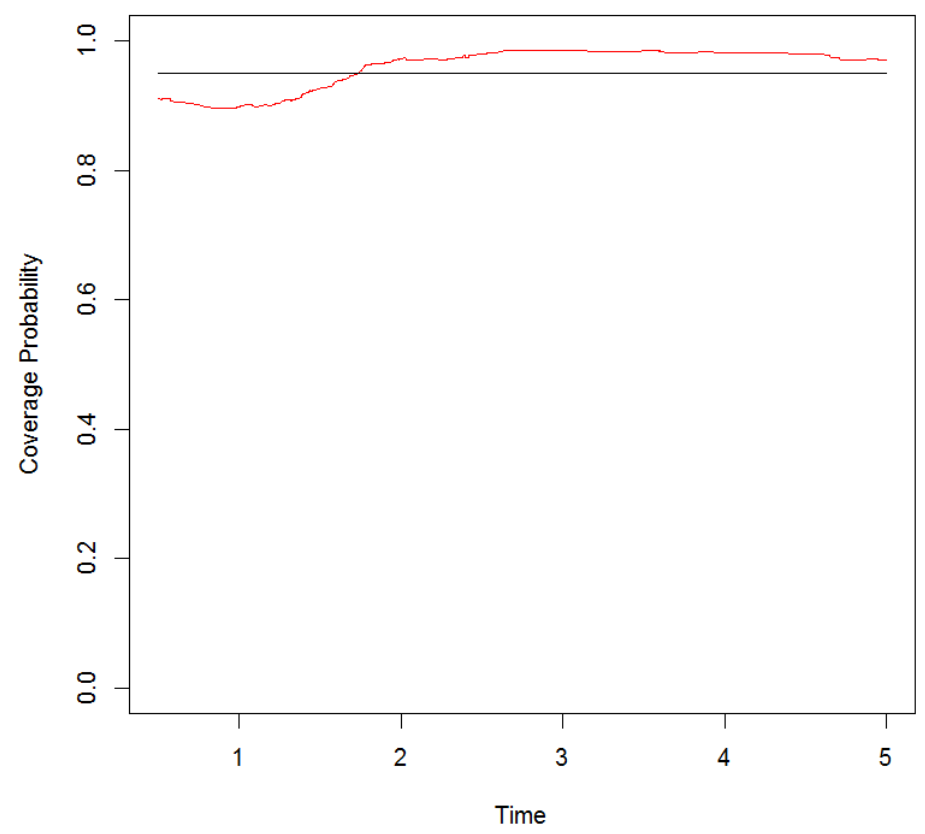

Figure 13: Coverage probabilities for $\tilde{q}$ in the case with 150 individuals and 10 time panels

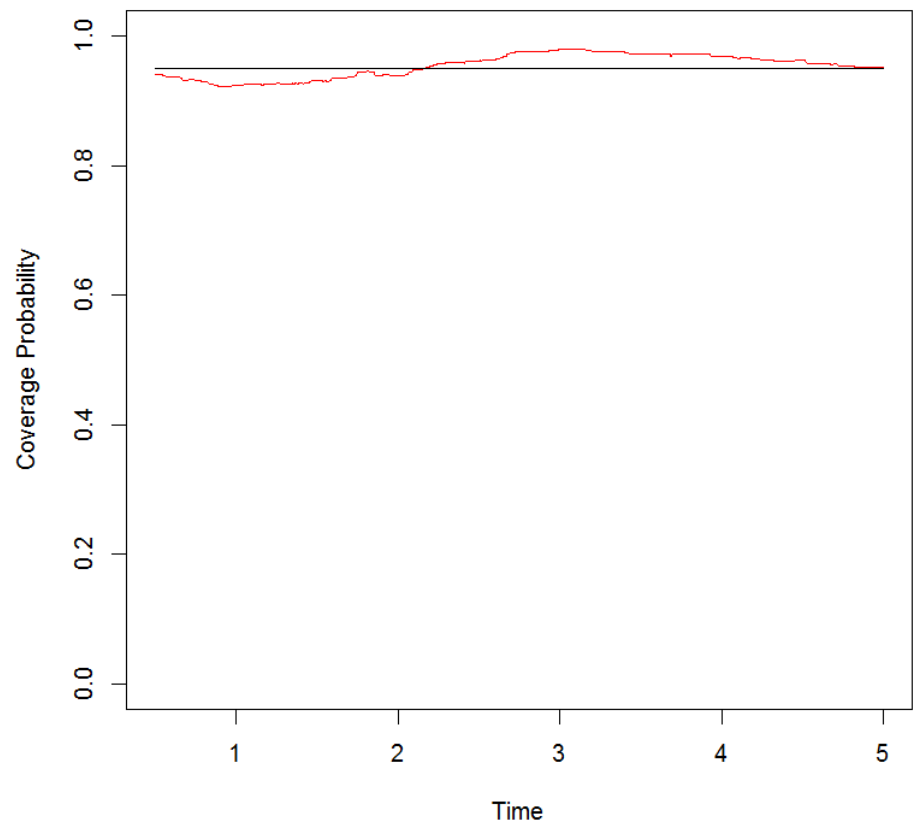

Figure 14: Coverage probabilities for $\tilde{q}$ in the case with 500 individuals and 50 time panels 
The coverage probabilities for all cases are around 0.95 since the fit is good and there is sufficient data. The remaining coverage probability graphs can be found in the appendix.

In summary, fitting a data set with a known underlying structure using a modelbased penalty provides excellent fit results. It gives the modeler options, as the modelbased penalty term can be adjusted to include the underlying function of the data. As long as $\lambda$ is sufficiently large, it will force the model to converge at that function. Also, this scenario provides the smallest possible bias and standard error as the penalty term essentially computes maximum likelihood estimates for the function's parameters. Maximum likelihood estimates in turn have the lowest possible bias and standard error of all estimates for a parameter. In order to determine if an underlying structure exists, it is recommended that the modeler first inspect the data and potential fits to ensure that their assumption is correct. In certain scenarios, such as survival analysis, common underlying structures are known to exist and thus this model would be more effective than a more flexible approach, such as a non-penalized spline.

\subsection{Fitting a non-Weibull Function}

The other function chosen for the majority of the simulations run was a quadratic function. The data generated from the quadratic function chosen could not be fit using a Weibull hazard. This was done in the hopes of demonstrating how the proposed model can still provide reasonable fits, even in an entirely unreasonable scenario. However, it is important to understand that fitting a quadratic function under the assumption of an underlying Weibull function (or any other function that clearly will not model the data well) is not a recommended approach. Since the penalty is always present, it will influence the fit and attempt to steer results towards the function used. We now explore the graphical output from the simulations to understand where this approach works and fails. We will then discuss a more optimal approach for this case in the next section.

In the ideal case, the quadratic fit is smooth and sits fairly close to the true function, as seen in Figure 15. However, the fit is clearly not the best that can be obtained from 


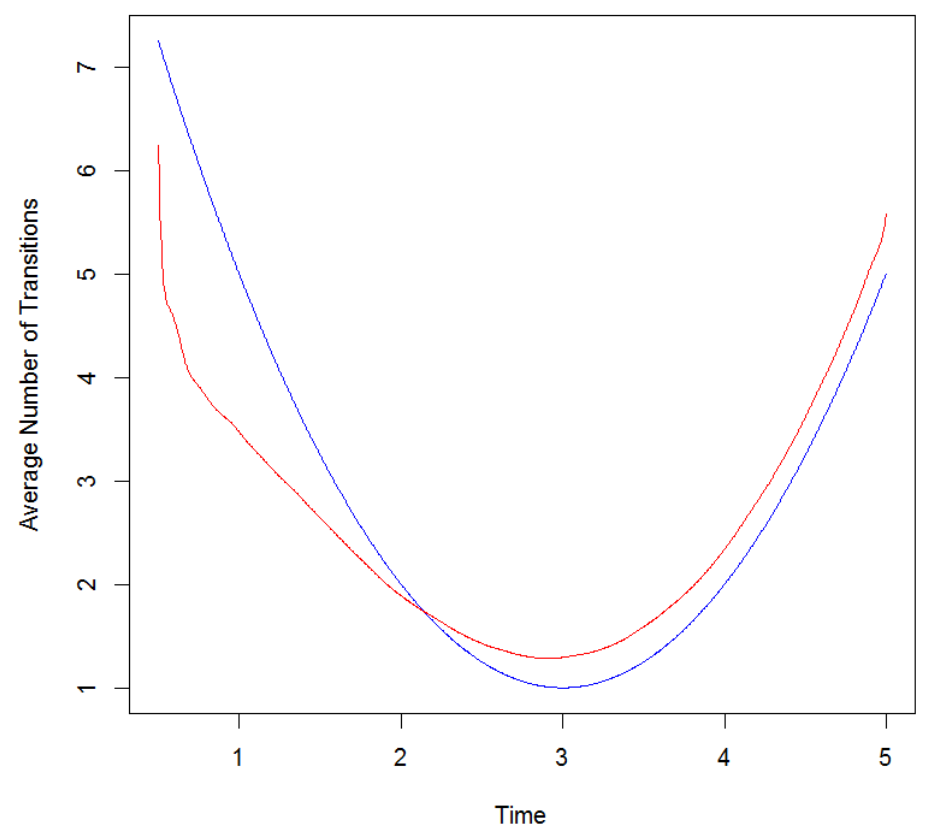

Figure 15: Quadratic fit for the case with $n=500$ and $l=50$

known methods.

Furthermore, it becomes increasingly evident as we examine simulations with fewer individuals and time panels that the model breaks down quite quickly. For example, in Figure 16 we see the fit with $n=250$ and $l=25$. As time gets smaller, the spline struggles to stabilize. This indicates that the gradient of the fit does not remain consistent, causing the spline to become wavy. At the far left end, where there may be less information due to the data that were generated, the spline is being forced to infinity, resulting in an asymptote. Reducing the number of individuals and time panels further gives even worse results, as shown in Figure 17. The function becomes a poorer fit throughout time and much less smooth. While some of the change can be attributed to reduction of data, it is important to note that the penalty term is trying to force the quadratic data to a Weibull hazard function. Evidently, this is not working well. This increase is variation could be due to the fact that a larger number of knots (here we used 20 ) is being used when the penalty term is not having a great influence on outcome. So, 
the spline is showing more variation than actually exists in the data.

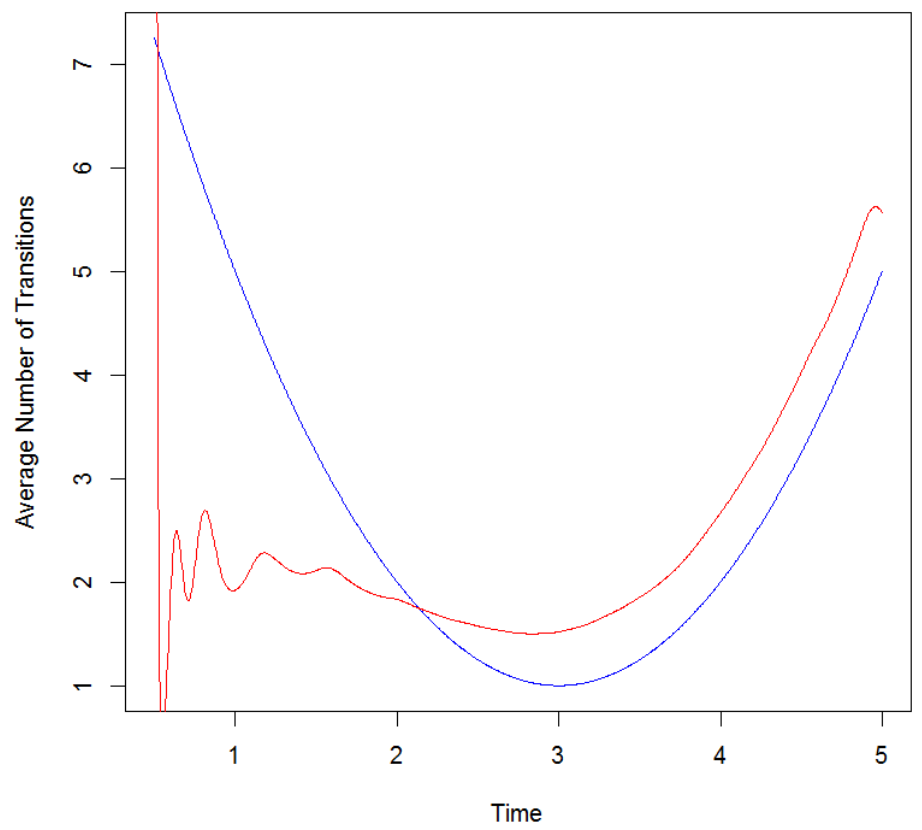

Figure 16: Quadratic fit for the case with $n=250$ and $l=25$ 


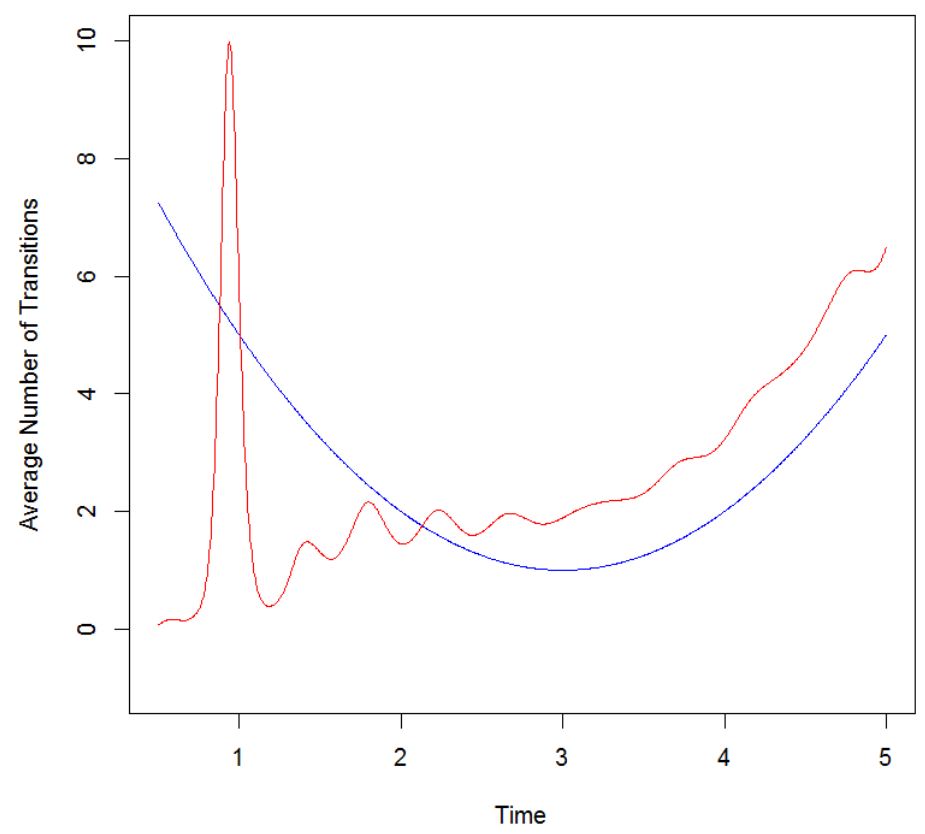

Figure 17: Quadratic fit for the case with $n=150$ and $l=10$

As expected, bias results for this function were not optimal. They did however, remain fairly small where the fit was more smooth, as shown in Figure 18. Figure 19 demonstrates that once the function starts to fail, the bias increases substantially. For the quadratic function, it appears that the bias is lower as the number of panels increases. It still remains consistently large at smaller times, but at the times where the fit is reasonable, an increase in the number of panels lowers bias. This may be due to the fact that the spline uses a finer grain to model the data, meaning that more cubic spline segments are used in total, and thus it does not have the opportunity to increase or decrease dramatically.

Last, we must evaluate coverage probabilities. Due to the model's difficulty in fitting this scenario, the coverage probabilities over time are extremely inconsistent. They do tend to increase as time increases because the model performs better at later times. This may be explained by the variance of the model at various times and the sparser data present at earlier times due to the generated data. Since the coverage probabilities for 


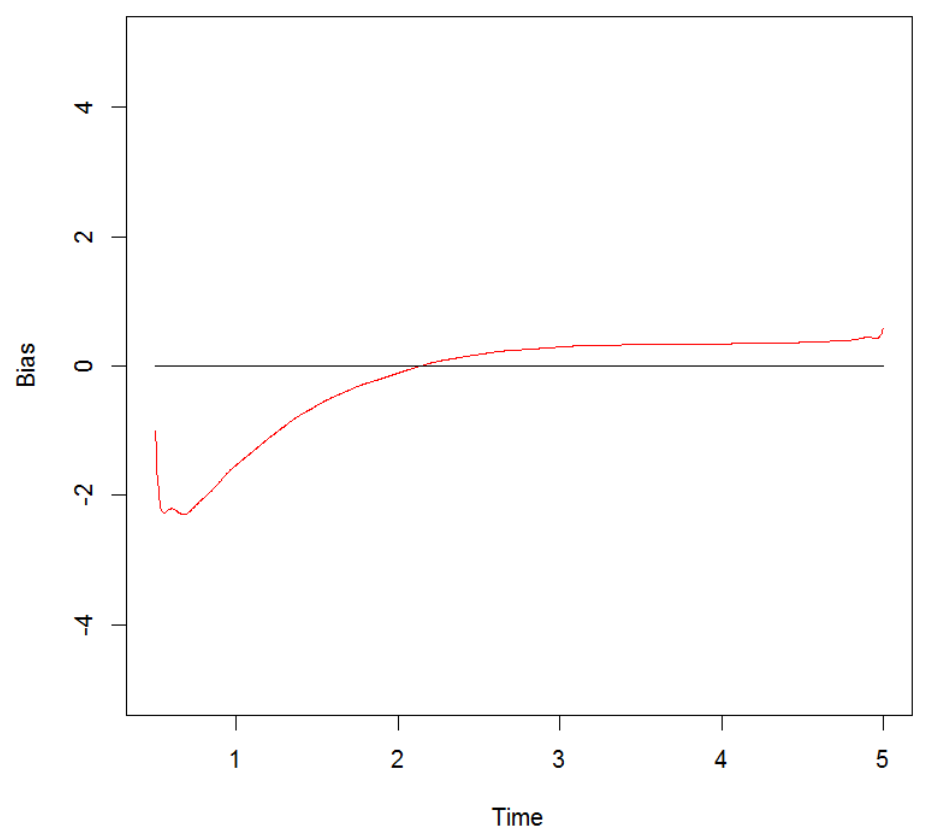

Figure 18: Bias for the case with $n=500$ and $l=50$

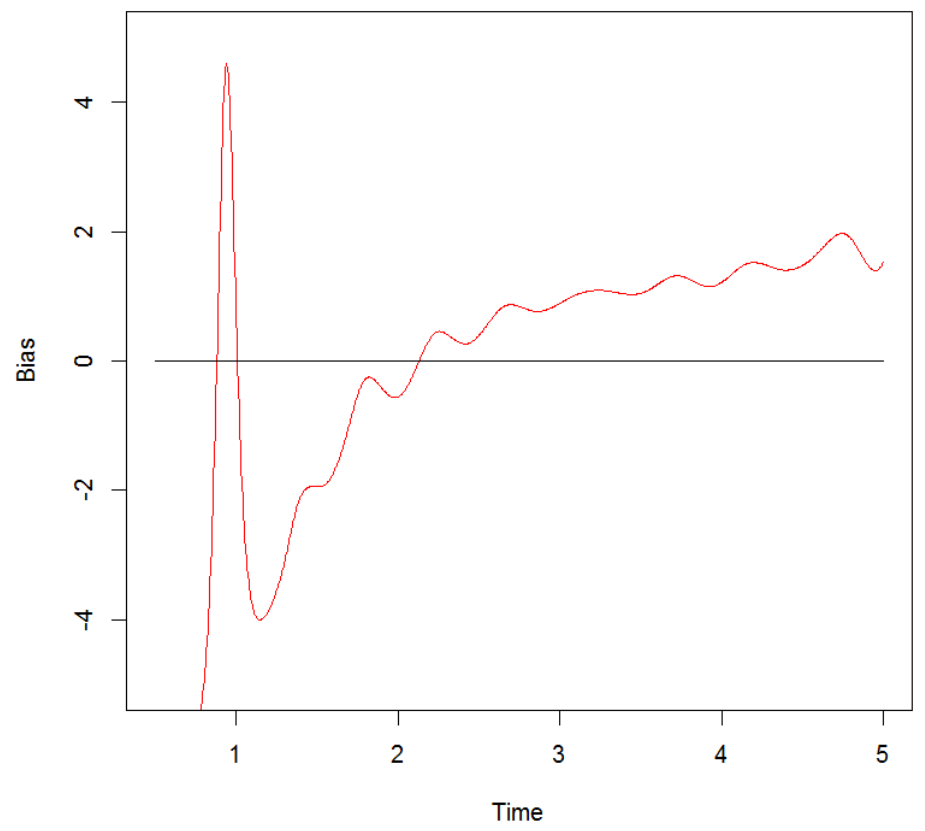

Figure 19: Bias for the case with $n=250$ and $l=10$ 


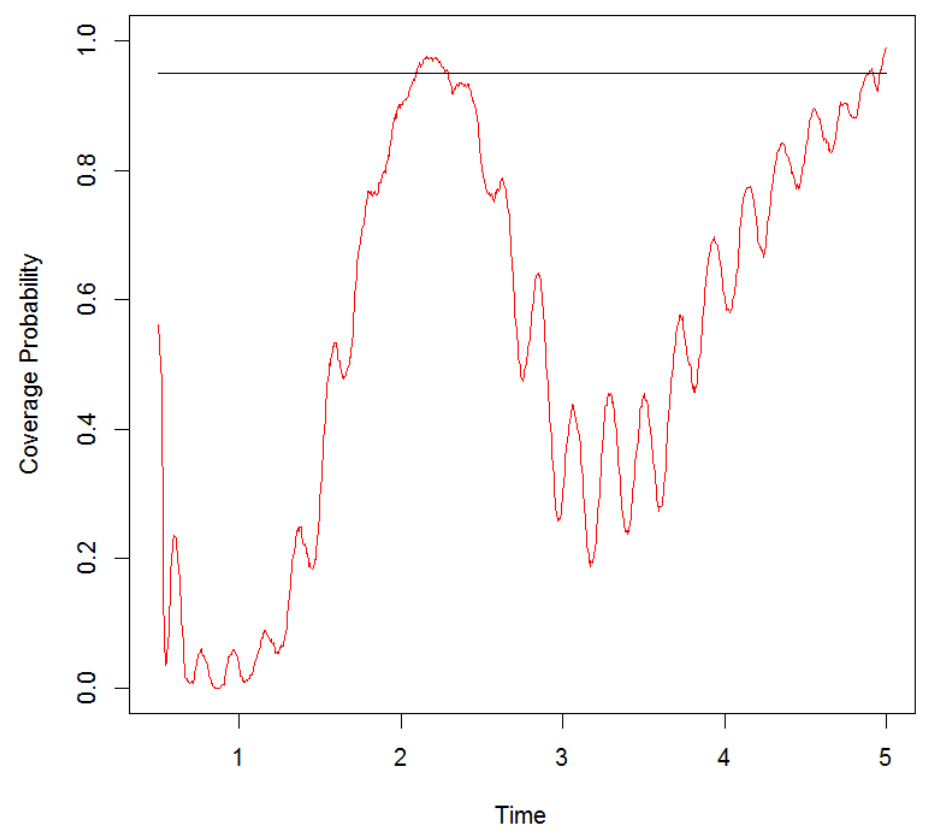

Figure 20: Coverage probabilities for the case with $n=500$ and $l=50$

the quadratic function were calculated using all portions of the $M$ matrix and the Delta Method, the data influences variance significantly. This leads to increased standard error where the data does not line up with the direction that the penalty term wants to take the fit. This phenomenon is clearly evidenced in the coverage probability plot for the $n=500$ and $l=50$ case in Figure 20. The coverage probability plots for the other cases are significantly more inconsistent. They can be examined in the Appendix.

So, we conclude this section with the understanding that a model-based penalty that has absolutely no relation to the underlying structure of the data will not perform well. The fits obtained are mediocre at best and there are likely many more options that would fit the data with much less error and bias. We now evaluate one potential option for obtaining a better fit in cases such as this. 


\subsection{Using a Second Derivative Penalty for the non-Weibull function}

As shown in the previous section, modeling a transition intensity using an inappropriate penalty term does not produce desirable results. We recommend that instead a second derivative penalty (or an approximation to the second derivative) is used for the transitions where an underlying distribution structure does not exist. This can be done simultaneously with a model-based penalty; the modeler can chose which penalty format to use depending on their apriori knowledge of the data. Preliminary inspection of the data and some investigative fitting should be able to suggest the best route for the penalty term. In the simulation run for this case, a divided difference penalty was used instead of the model-based penalty for the quadratic function. This penalty approach can be used since we have equally spaced time points. The divided difference penalty is attractive because it simplifies many calculations since it does not require the computation of any derivatives. All other parameters remain fixed at the same values. That is, $n=500, l=50$ and $\lambda=11$.

Figure 21 demonstrates the quadratic fit using the second derivative penalty as opposed to the model based penalty. We see that the fit is much more appropriate and is reasonably smooth due to the influence of the penalty term. Clearly, when comparing this fit to the fit using the model-based penalty, a modeler would definitely prefer the divided difference penalty. That being said, there is also room for experimentation with the type of penalty used. If a different type of second derivative penalty is preferred, such as the integral of the squared second derivative of the function, it may also be used. Essentially, the flexibility in penalty type can be used to 'mix and match' penalties for different transition intensities in a single transition intensity matrix.

As may be inferred from the great improvement in fit using this penalty, there was also substantial improvement in the bias and coverage probabilities over time. A quick inspection of Figures 22 and 23 shows that this fit has very low bias and coverage probabilities close to the expected 0.95 level.

We now consider an intermediate case with a function that is not the same as the 


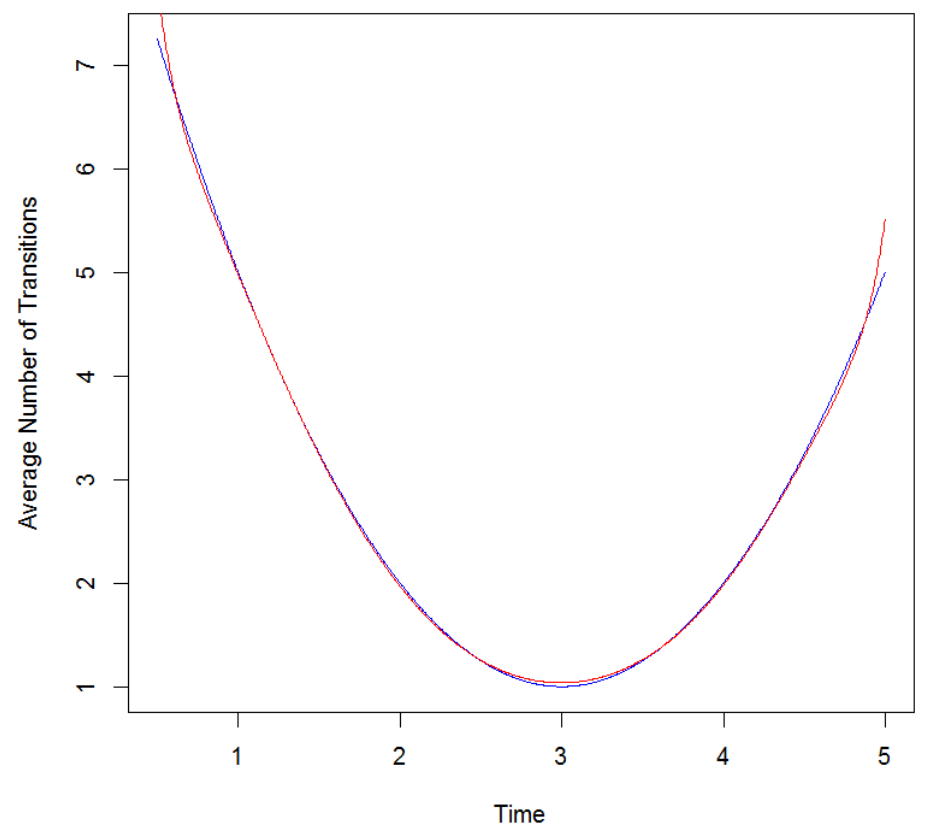

Figure 21: Quadratic fit using the divided difference penalty

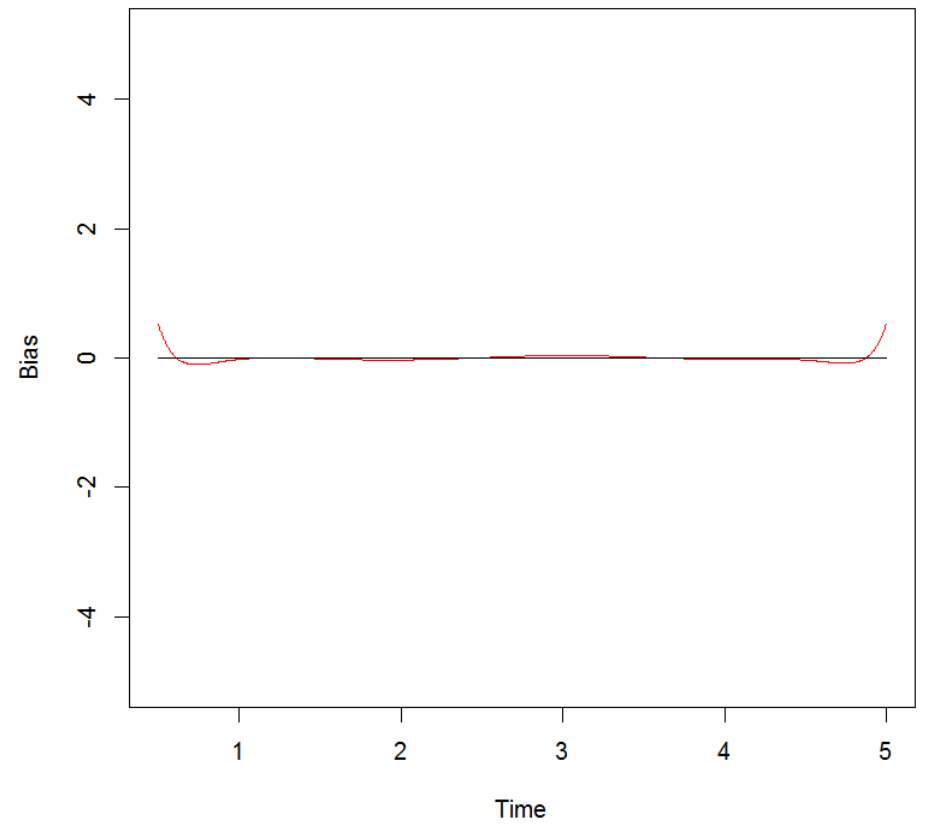

Figure 22: Quadratic bias using the divided difference penalty 


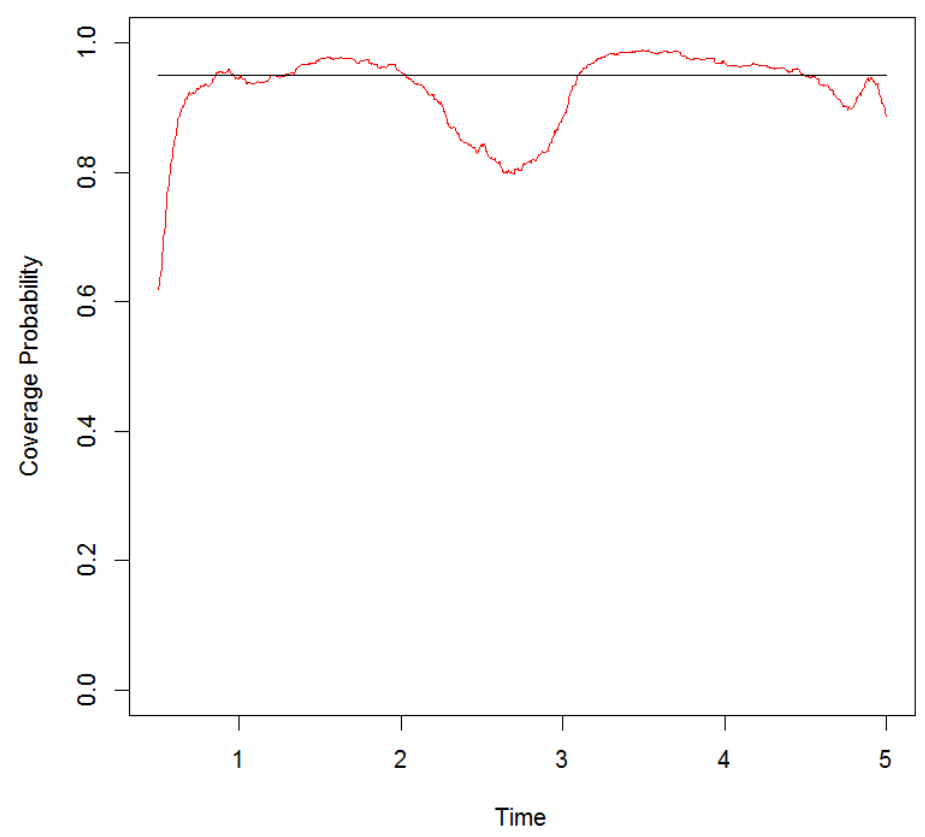

Figure 23: Coverage probabilities for the quadratic fit using the divided difference penalty

function used in the model-based penalty, but that still fits well.

\subsection{Using a Log-Normal Function with the Model-Based Penalty}

Having seen an ideal scenario and an undesirable one, it is important to now consider an intermediate case. In order to better asses the model's performance, we used data generated from a log-normal probability distribution and attempted to fit it using the model with a Weibull penalty term. Though the log-normal is clearly not a Weibull function, the two have roughly similar shapes and thus the influence of the penalty term leads to a close, smooth fit.

We first consider the overall fit of the function with $\lambda=40$ and log-normal parameters $\theta=0, m=2$ and $\sigma=1.5$. The parameter $\lambda$ was set to be larger than in any quadratic case in order to increase the power of the penalty term in driving model fit. Seeing as a Weibull hazard function can fit a log-normal function relatively well, it is important to take advantage of such an option. Figure 24 demonstrates how this works. 


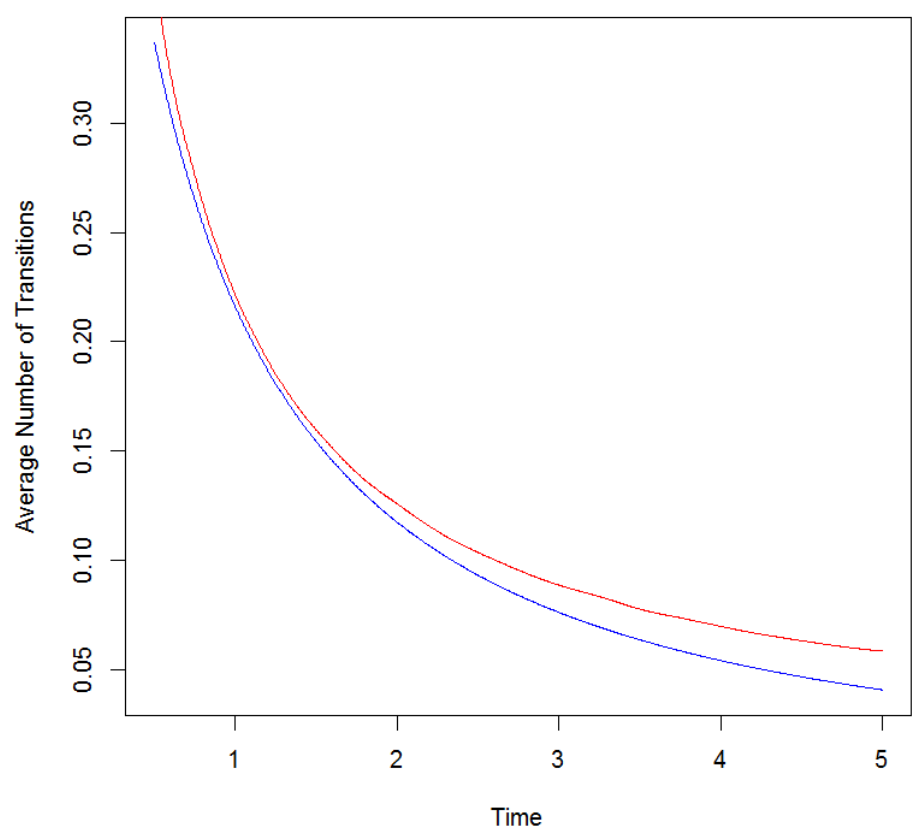

Figure 24: Log-normal function fit using the Weibull penalty

We clearly see that at lower times, where the log-normal probability distribution and Weibull hazard function are more similar, the fitted line (red) is closer the the true value of the log-normal function (blue). As time increases, the influence of the penalty term begins to force the tail end of the fit towards a Weibull hazard function as opposed to remaining true to the original function. We also note the smoothness of the function as the penalty term smooths any waves the spline may be considering. In the bias plot in Figure 25, we see that the bias is nearly constant throughout and increases slightly as time increases. This matches what we can determine visually from the fit plot. 


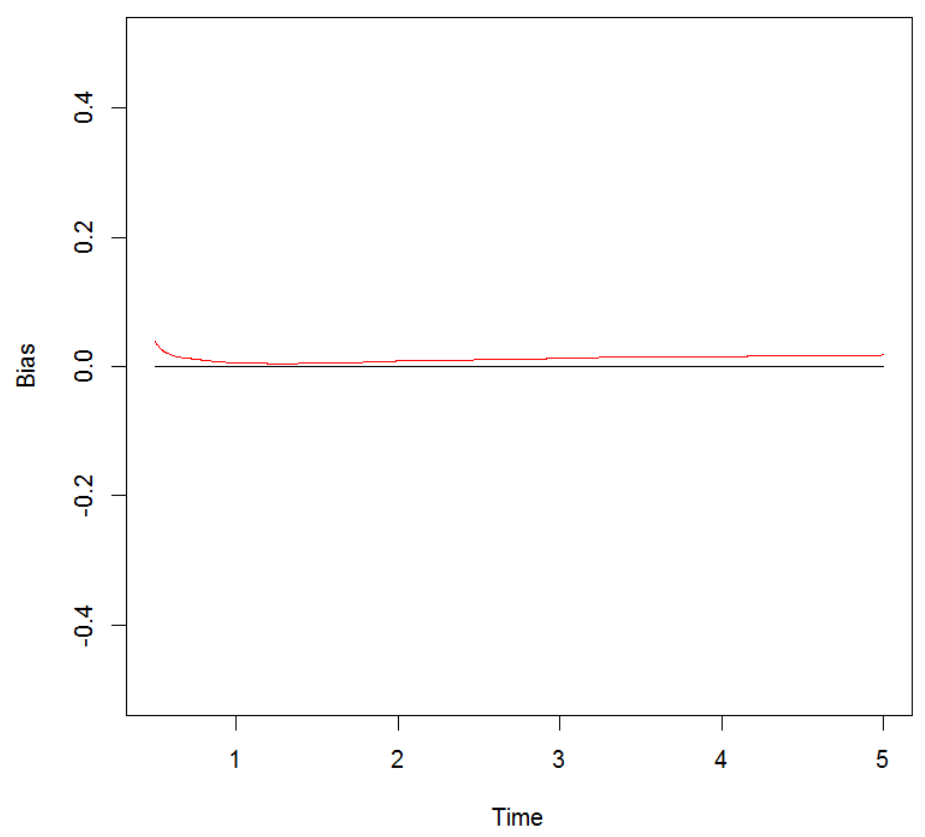

Figure 25: Log-normal function bias using the Weibull penalty

Finally, in the plot of coverage probabilities, we see that the coverage probabilities remain reasonable where the function fits well. Then, where the fit beings to fail, we see a clear decrease in coverage probabilities. This section of the plot is in line with where the bias increases slightly and the fit does not perform as well, so it is possible that in these later times the model is not the best choice at later time points.

Though this fit is not as perfect as the Weibull function or the quadratic function using the divided difference penalty, it does demonstrate the potential of the model-based penalty in fitting similar functions. In future attempts, one might consider more optimal methods of choosing a $\lambda$ value or even a dynamic way to determine which Weibull hazard parametrization is the closest match to the data generated. At this time, these values were chosen using repeated simulations and averaging over the values used in runs that performed as desired. We now discuss some potential options for choosing $\lambda$. 


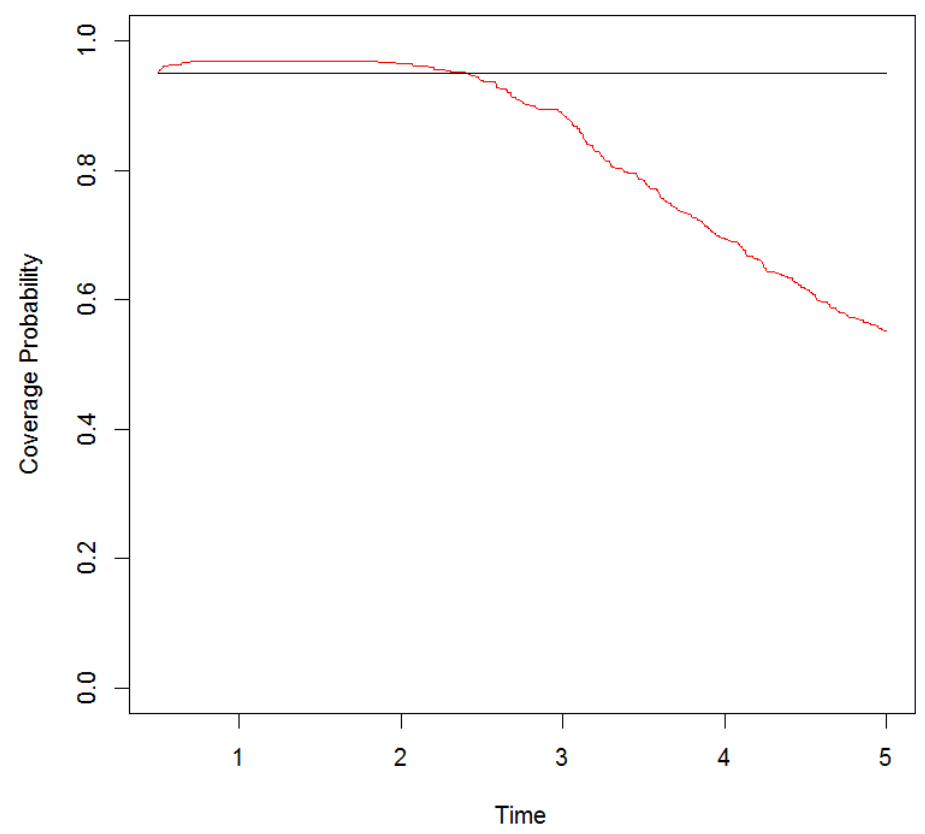

Figure 26: Log-normal function coverage probabilities using the Weibull penalty

\subsection{Optimizing $\lambda$}

For the simulation presented in this thesis, $\lambda$ was chosen experimentally in order to reduce simulation times. Smaller sample runs were done starting with large $\lambda$ values and scaling them down until an appropriate fit for the non-Weibull scenario was found. More reliable and precise methods to find a $\lambda$ value exist and will be discussed here. These methods would give the best possible $\lambda$ value for each simulation scenario, but as these methods require fitting the model numerous times per simulation, it increases the time required to obtain results.

\subsubsection{Generalized Cross Validation}

One possible option for choosing $\lambda$ dynamically is generalized cross validation or GCV. GCV is commonly used to minimize the mean squared error (MSE) of a linear model fit [6]. It is considered a desirable method for finding the MSE because it does not require the computation of an estimate for the variance of a model, which can sometimes be a 
computationally costly procedure 15 . Also, GCV handles outliers better than ordinary cross validation[15]. Suppose now that we can write

$$
\hat{f}=\mathbf{S Y}
$$

where $\mathbf{S}$ is given by $\mathbf{S}=\mathbf{X}\left(\mathbf{X}^{\mathbf{T}} \mathbf{X}+\lambda \mathbf{P}\right)^{-1} \mathbf{X}^{\mathbf{T}}$ and trace $(\mathbf{S})$ represents the effective degrees of freedom of the model. $\mathbf{X}$ represents the design matrix and $\mathbf{P}$ represents the penalty. As shown earlier, $\mathbf{X}$ can be replaced by $\mathbf{B}$ when using B-Splines. Then the GCV is given by

$$
G C V(\hat{f})=\frac{(\mathbf{Y}-\hat{\mathbf{f}})^{T}(\mathbf{Y}-\hat{\mathbf{f}})}{n-\operatorname{trace}(\mathbf{S})}
$$

In the context of finding a suitable $\lambda$ value for the model presented in this project, this GCV calculation would be run numerous times with varying values of $\lambda$. The $\lambda$ that gives the smallest GCV value for each data set would be chosen as the optimal $\lambda$.

\subsubsection{Restricted Maximum Likelihood}

Another potential option for choosing an optimal $\lambda$ value for each data set is restricted maximum likelihood, or REML. REML variance estimates provide smaller bias than maximum likelihood estimates [2], though in many cases the two methods provide similar results. In order to find an optimal $\lambda$ for the model above, we must use a fixed point algorithm on the ratio between the overall variation for the REML fit and the variation captured by the spline. The overall variation for the REML fit is given by

$$
V(\lambda)=\frac{(\mathbf{Y}-\hat{\mathbf{f}})^{T} V^{-1}(\mathbf{Y}-\hat{\mathbf{f}})}{n-e d f(\lambda)}
$$

where $V$ is the variance and $e d f(\lambda)$ is the effective degrees of freedom in the model. The variation captured by the spline is given by

$$
\tau(\lambda)=\frac{\mathbf{\Psi}^{T} \mathbf{P} \boldsymbol{\Psi}}{e d f(\lambda)}
$$


Thus the fixed point algorithm gives

$$
\lambda_{i+1}=\frac{V\left(\lambda_{i}\right)}{\tau\left(\lambda_{i}\right)}
$$

which will always converge, but not necessarily to a unique value [2]. Once we have many possible $\lambda$ values, we can take each of them and find the REML estimator for our function. The $\lambda$ that maximizes the REML estimator is the optimal $\lambda$.

\subsection{Extension to Covariates}

In future work, it may be of interest to consider the addition of covariates into the model. To incorporate a vector of covariate parameters $\underline{\beta}$ we first define $\underline{\theta}=\left[\underline{\boldsymbol{\Psi}}^{T}, \underline{\beta}^{T}\right]^{T}$ where $\underline{\boldsymbol{\Psi}}$ is the vector of spline coefficients as before and $\underline{\beta}$ is the vector of covariate parameters.

Let $D_{i j l}$ be a set of individuals $\left(n_{i j l}\right)$ who experience a transition from state $i$ to state $j$ in the $l^{t h}$ panel $\left(t_{l-1}, t_{l}\right]$. The likelihood is then given by

$$
\mathcal{L}(\underline{\theta})=\prod_{l=1}^{m} \prod_{i=1}^{k} \prod_{j=1}^{k} \prod_{v \in D_{i j l}} p_{i j v}\left(t_{l-1}, t_{l} ; \underline{\theta}\right) .
$$

Here,

$$
\begin{aligned}
\mathbf{P}_{v}\left(t_{l-1}, t_{l} ; \underline{\theta}\right) & =e^{\mathbf{z}_{v}\left(t_{l-1}, t_{l} ; \underline{\theta}\right)} \\
& =\left\{p_{i j v}\left(t_{l-1}, t_{l} ; \underline{\theta}\right)\right\}_{k \times k}
\end{aligned}
$$

where

$$
\begin{aligned}
\mathbf{Z}_{v}\left(t_{l-1}, t_{l} ; \underline{\theta}\right) & =\int_{t_{l-1}}^{t_{l}} \mathbf{Q}_{v}(u ; \underline{\theta}) d u \\
& =\left\{\int_{t_{l-1}}^{t_{l}} q_{i j v}(u ; \underline{\theta}) d u\right\}_{k \times k}
\end{aligned}
$$

Next, we consider the intensity under the standard proportional hazard assumption, which is used for recurrent events [17]. Under a proportional hazards or intensity 
assumption,

$$
q_{i j v}(t ; \underline{\theta})=q_{0 i j}(t ; \underline{\Psi}) e^{\underline{x}_{v} \underline{\beta}_{i j}}
$$

where $\underline{x}_{v}=\left[x_{v 1}, \ldots, x_{v p}\right]^{T}$ is a vector of covariates for the $v^{t h}$ individual or object in the study and

$$
q_{0 i j}(t ; \underline{\mathbf{\Psi}})=e^{\underline{\mathbf{B}}(t)^{T} \Psi_{i j}}
$$

as defined in the thesis. This means that

$$
\int_{t_{l-1}}^{t_{l}} q_{i j v}(u ; \underline{\theta}) d u=e^{\underline{x}_{v}} \underline{\beta}_{i j} \int_{t_{l-1}}^{t_{l}} q_{0 i j}\left(u ; \Psi_{i j}\right) d u
$$

and we can proceed as was done previously in order to find estimates for the $\underline{\boldsymbol{\Psi}}$ parameters and any subsequently derived quantities. 


\section{Conclusion}

A penalized spline approach using a model-based penalty has been shown to work effectively in cases where the underlying structure of the data follows the same distribution as the one used in the model-based penalty. This provides a method for fitting data using splines and a penalty term that, when suitable, can lead to the the data being fit with essentially maximum likelihood estimates. When the penalty term has a greater influence, this modeling approach will give better fits providing the underlying data follows the model-based penalty. Hence, this method is appropriate for fitting functions that are known to have an apriori underlying structure and those that are similar. Conversely, the method does not provide reasonable fits for functions that are not close to the underlying assumption. In such cases, the roughness parameter $\lambda$ can be kept low in order to allow the spline component of the model to drive the fit. Also, the penalty term can be replaced with a second derivative penalty, in cases where the fit is found to be very reasonable.

There is great potential for future work on this modeling approach. The first is to use an iterative method to select the most appropriate $\lambda$ value for each data set. This can be done using methods such as GCV and REML. Furthermore, in the case of a very large $\lambda$ value, different methods should be considered for finding the variance and standard error of the model. Sampling methods such a bootstrap or jackknife re-sampling would likely provide better results. Methods for choosing Weibull parameters (or the parameters of any model used in the penalty term), will need to be investigated to give the closest possible distribution to the current data. Repetitive sampling methods on non-linear regression may be considered in this case. Also, knot placement in the spline could be dynamic instead of at equally spaced intervals. Multiple procedures in $R$ exist to accomplish this.

Finally, using the approaches for a penalty term discussed in the thesis can be improved in order to choose the most appropriate penalty depending on the function, without the modeler having to assign a type of penalty. For instance, the penalty term 
could be modified to be a weighted average between a model-based penalty term, such as the Weibull intensity discussed in this thesis, and a divided difference penalty. Then, GCV could be used to determine the most appropriate parameter value for the penalty. Depending on this value, the penalty would use the model-based approach or the divided difference approximation. 


\section{References}

[1] Friedman, M., Furberg, C.D., DeMets, D.L. Fundamentals of Clinical Trials, fourth edition. Springer Science + Business Media, New York, NY. 2010.

[2] Diggle, P.J, Heagerty, P., Liang, K.Y., Zeger, S.L. Analysis of Longitudinal Data, second edition. Oxford University Press, Oxford, UK. 2013.

[3] Titman, A.C. Flexible Nonhomogeneous Markov Models for Panel Observed Data. Biometrics, Vol. 67 2011. pp.780-787

[4] Eilers, P.H.C., Marx, B.D. Flexible Smoothing with B-Splines and Penalties. Statistical Science, Vol.11, No.2. 1996. pp.89-121

[5] De Boor, C. On Calculating with B-Splines Journal of Approximation Theory, Vol.6 1972. pp.50-62

[6] Hastie, T., Tibshirani, R., Friedman, J. The Elements of Statistical Learning, second edition. Springer Science + Business Media, New York, NY. 2009.

[7] Kabfleisch, J.D., Lawless, J.F. The Analysis of Panel Data Under a Markov Assumption. Journal of the American Statistical Association, Vol.80, No. 392. Dec 1985. pp. $863-871$

[8] Heckman, N.E., Ramsay, J.O. Penalized Regression with Model-Based Penalties. The Canadian Journal of Statistics, Vol.28 No. 2. June 2000. pp.241-258

[9] Ramsay, J.O., Heckman, N.E., Silverman, B.W. Spline Smoothing with Model Based Penalties. Behavior Research Methods, Instruments and Computers, Vol.29 No.1. 1997. pp.99-106

[10] Klein, J.P., Moeschberger, M.L. Survival Analysis: Techniques for Censored and Truncated Data, second edition. Springer Science + Business Media, New York, NY. 2003. 
[11] Cox, D.R., Miller, H.D. The Theory of Stochastic Processes, first edition. John Wiley and Sons Inc, New York. 1965.

[12] Nelder, J.A., McCullagh,P. Generalized Linear Models, second edition. Springer Science + Business Media, New York, NY. 1989.

[13] Casella, G., Berger, R.L. Statistical Inference, second edition. Duxbury Press, Pacific Grove, CA. 2002.

[14] Prabhakar Murthy, D.N., Xie, M., Jiang, R. Weibull Models, first edition. John Wiley and Sons Inc, Hoboken, NJ. 2004.

[15] Golub, G.H., Heath, M., Wahba, G. Generalized Cross-Validation as a Method for Choosing a Good Ridge Parameter. Technometrics, Vol. 21 No. 2. 1979. pp. 215-223

[16] R Development Core Team (2008). R: A language and environment for statistical computing. R Foundation for Statistical Computing, Vienna, Austria. ISBN 3900051-07-0, URL http://www.R-project.org.

[17] Cook, R.J., Lawless, J. The Statistical Analysis of Recurrent Events. SpringerVerlag, New York, NY. 2007. 


\section{A Results Appendix}

Nine initial simulation cases were run, along with two subsequent cases to show an ideal scenario and to further demonstrate $\lambda$ 's influence. All figures obtained from the analysis will be displayed here case by case. The red lines represent the results obtained from the simulations: the fit, the bias and the coverage probabilities. Any blue lines represent the true function from which data were generated. Any black lines represent appropriate thresholds, such as zero bias or a confidence level of $95 \%$.

\section{A.1 Case 1: $n=500$ and number of time panels $=50$}

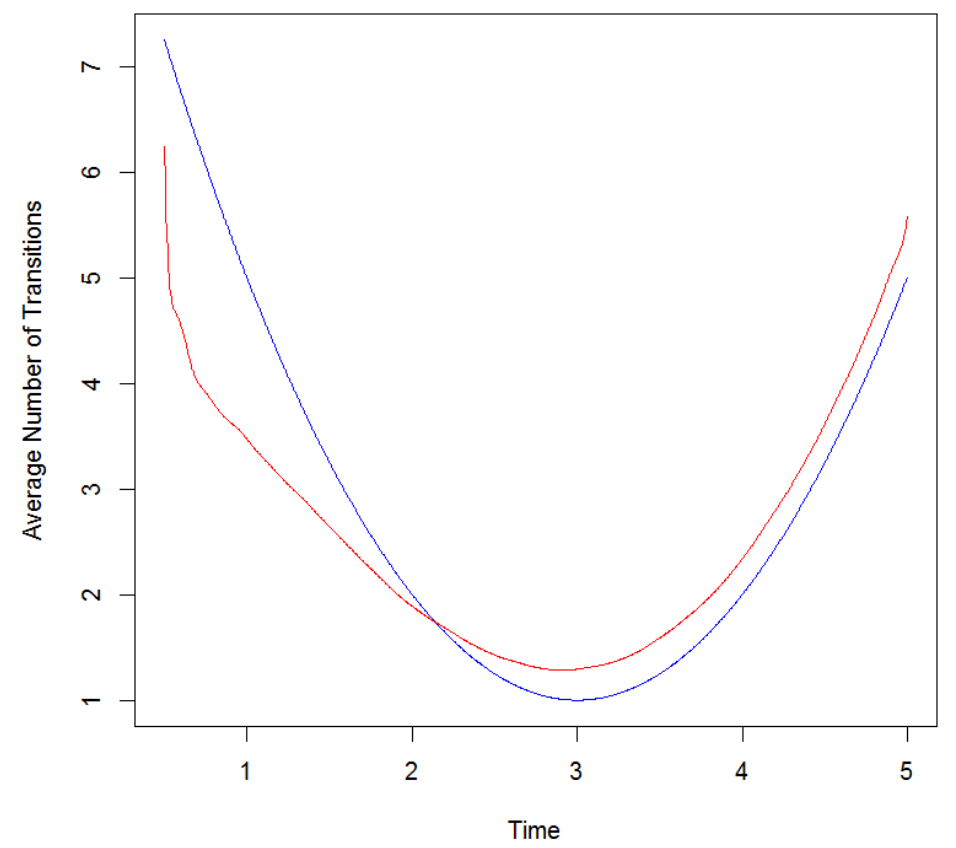

Figure A.1: Quadratic fit for Case 1 with $\lambda=11$ 


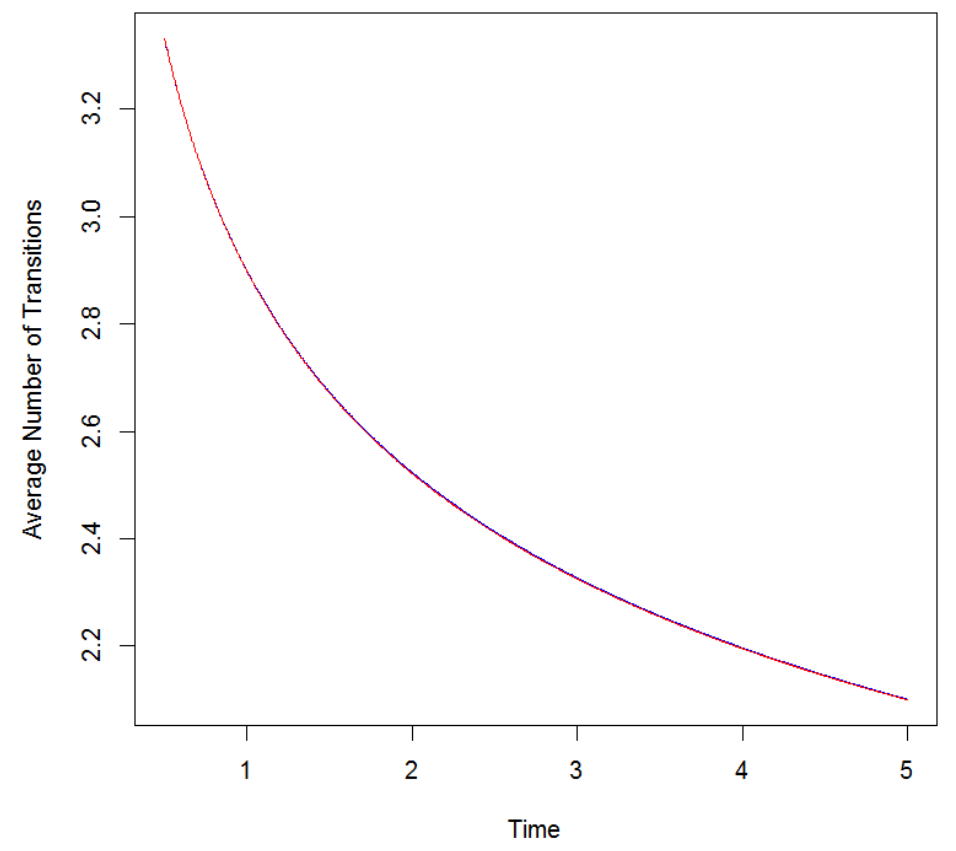

Figure A.2: Weibull fit for Case 1 with $\lambda=10000$

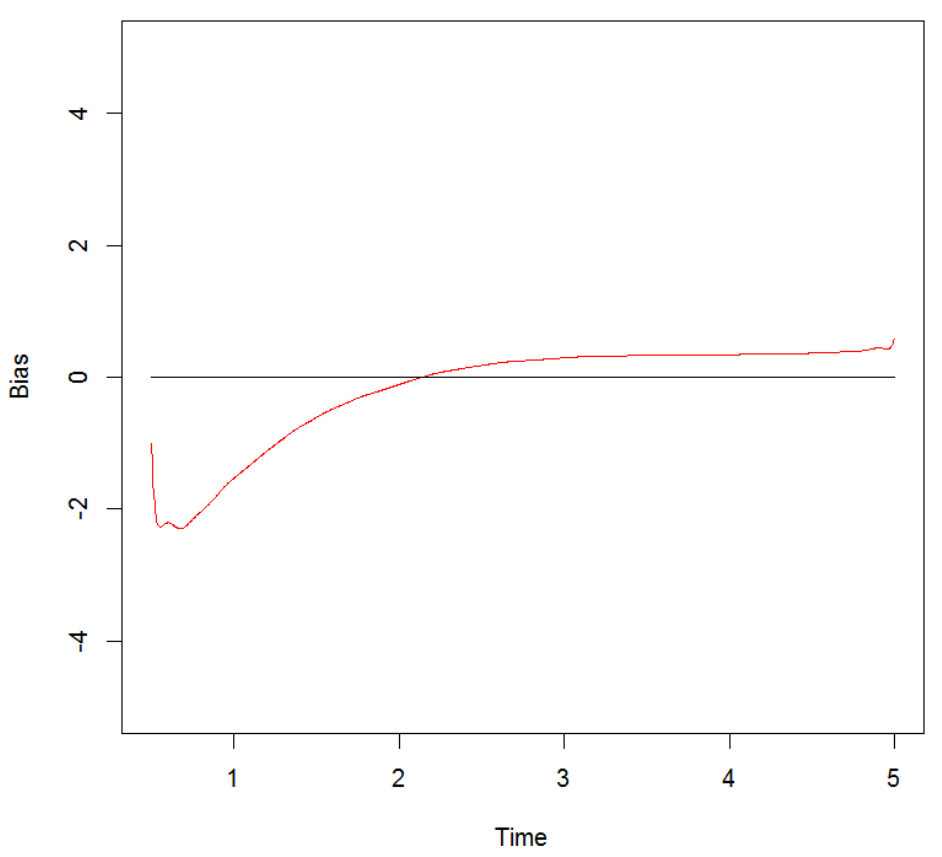

Figure A.3: Bias for the quadratic function of Case 1 


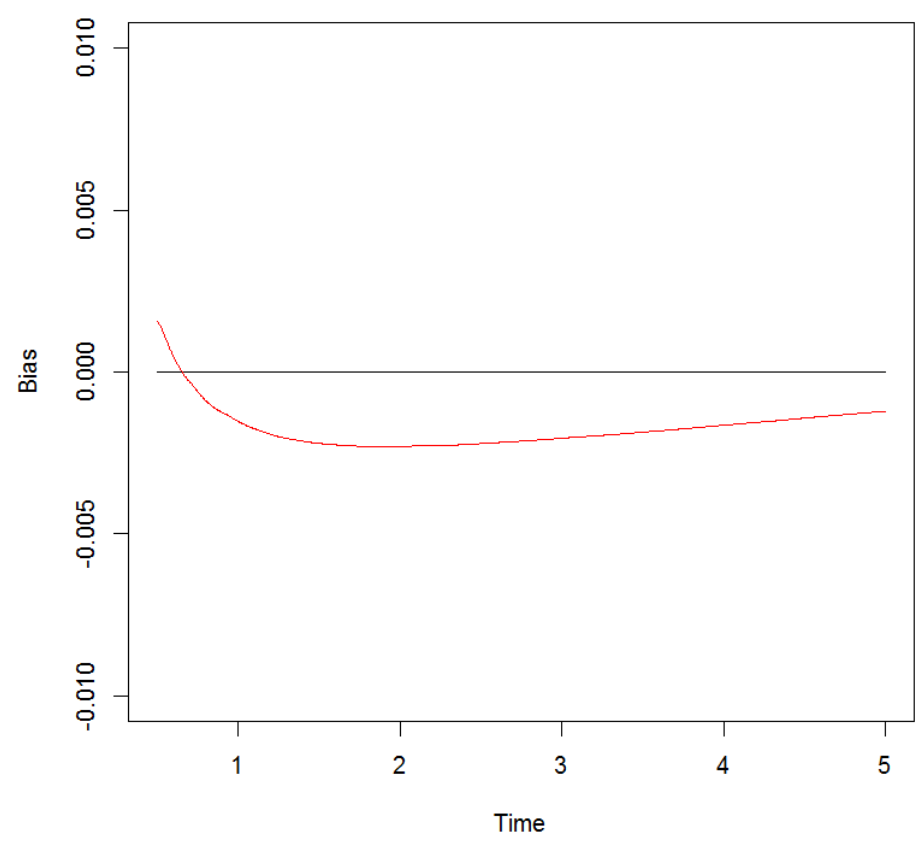

Figure A.4: Bias for the Weibull function of Case 1. We note that the graph is zoomed in significantly.

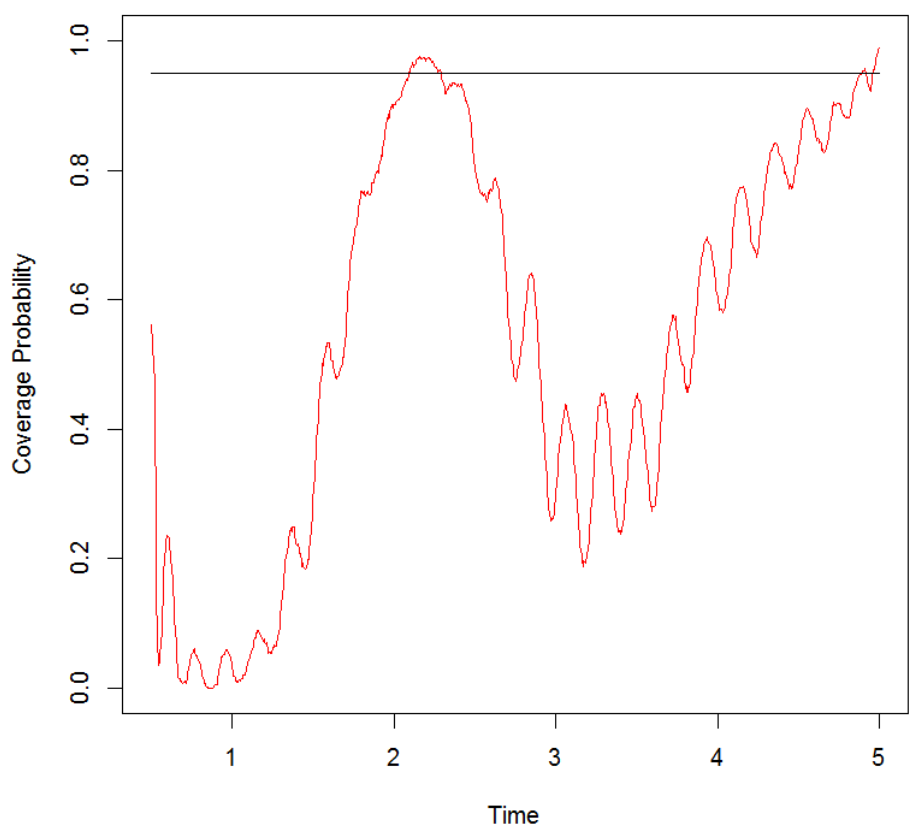

Figure A.5: Coverage probabilities for the quadratic function of Case 1 


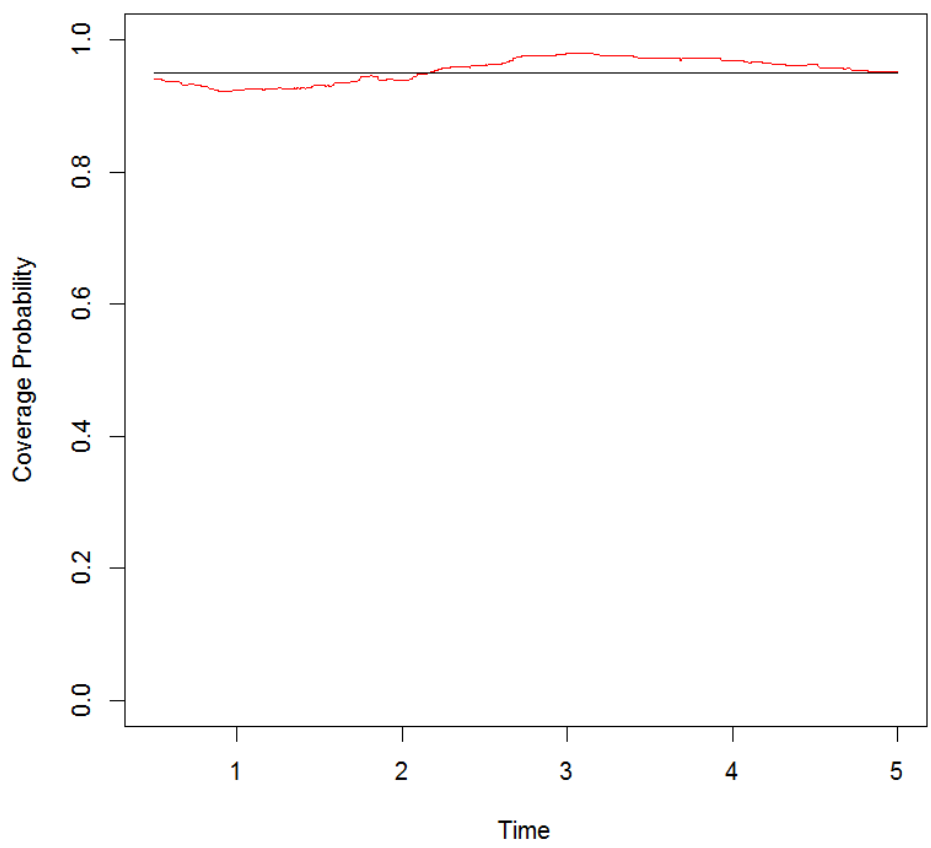

Figure A.6: Coverage probabilities for the Weibull function of Case 1 
A.2 Case 2: $n=500$ and number of time panels $=25$

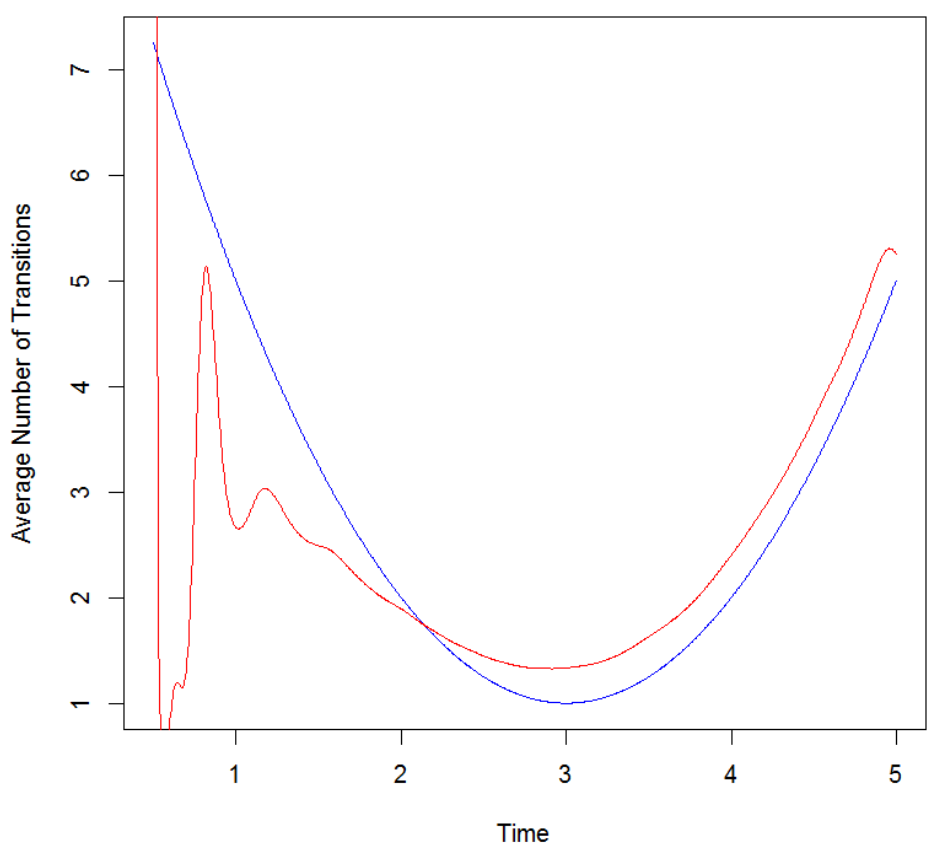

Figure A.7: Quadratic fit for Case 2 with $\lambda=11$ 


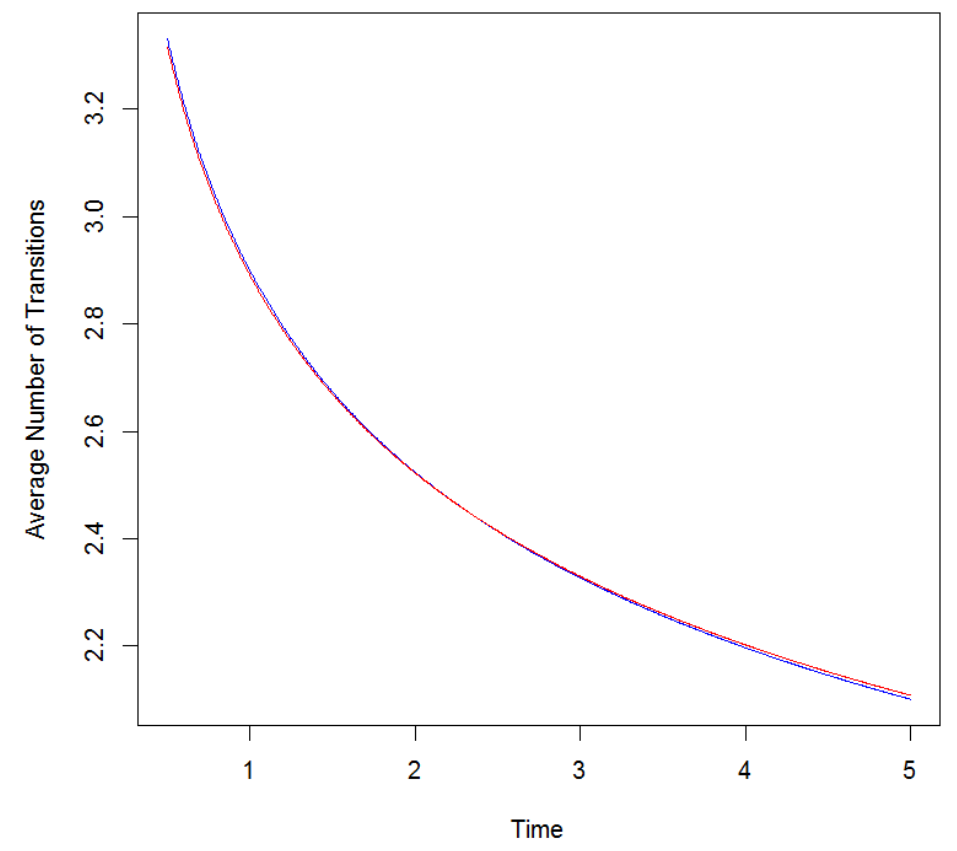

Figure A.8: Weibull fit for Case 2 with $\lambda=10000$

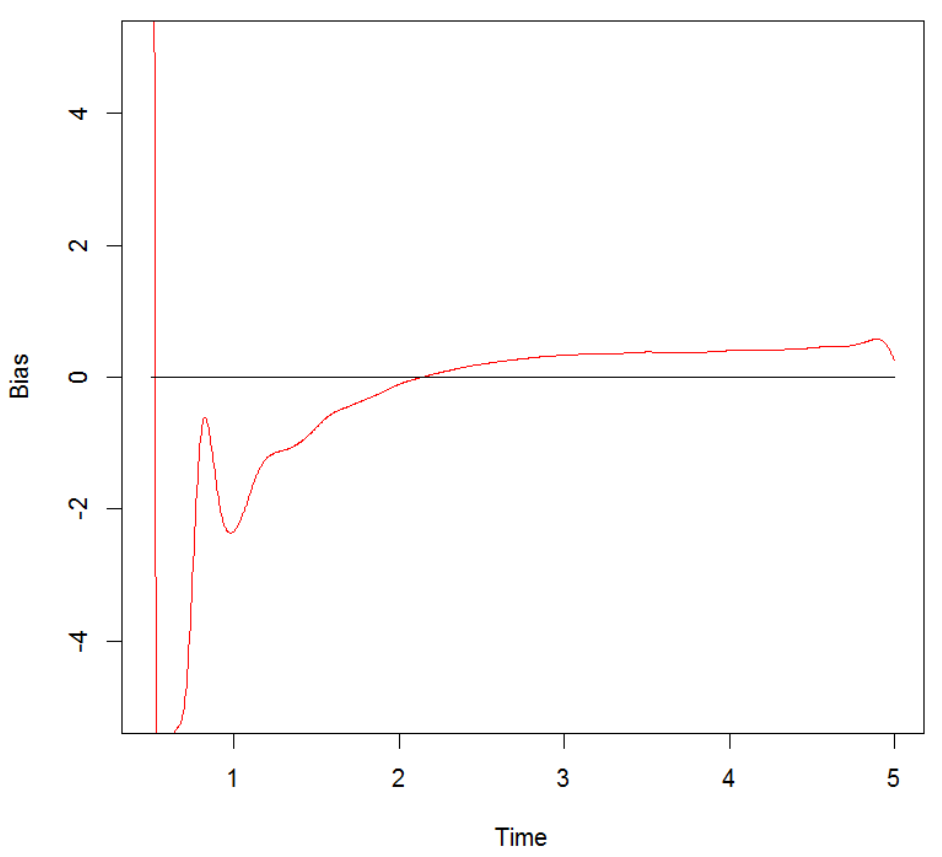

Figure A.9: Bias for the quadratic function of Case 2 


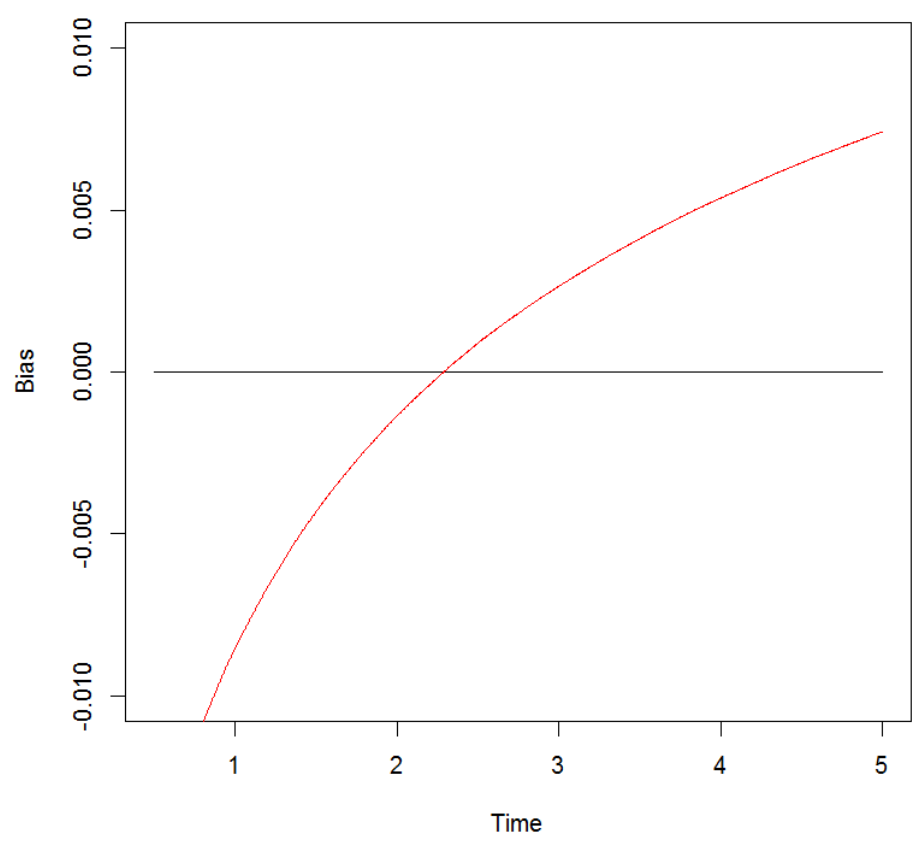

Figure A.10: Bias for the Weibull function of Case 2. We note that the graph is zoomed in significantly.

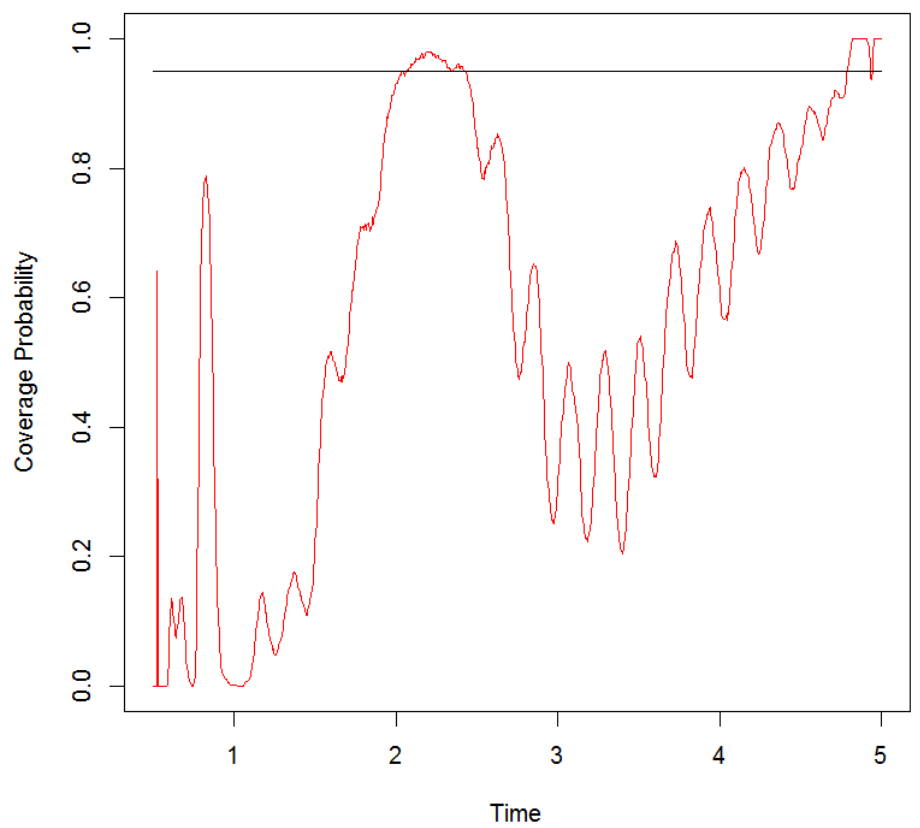

Figure A.11: Coverage probabilities for the quadratic function of Case 2 


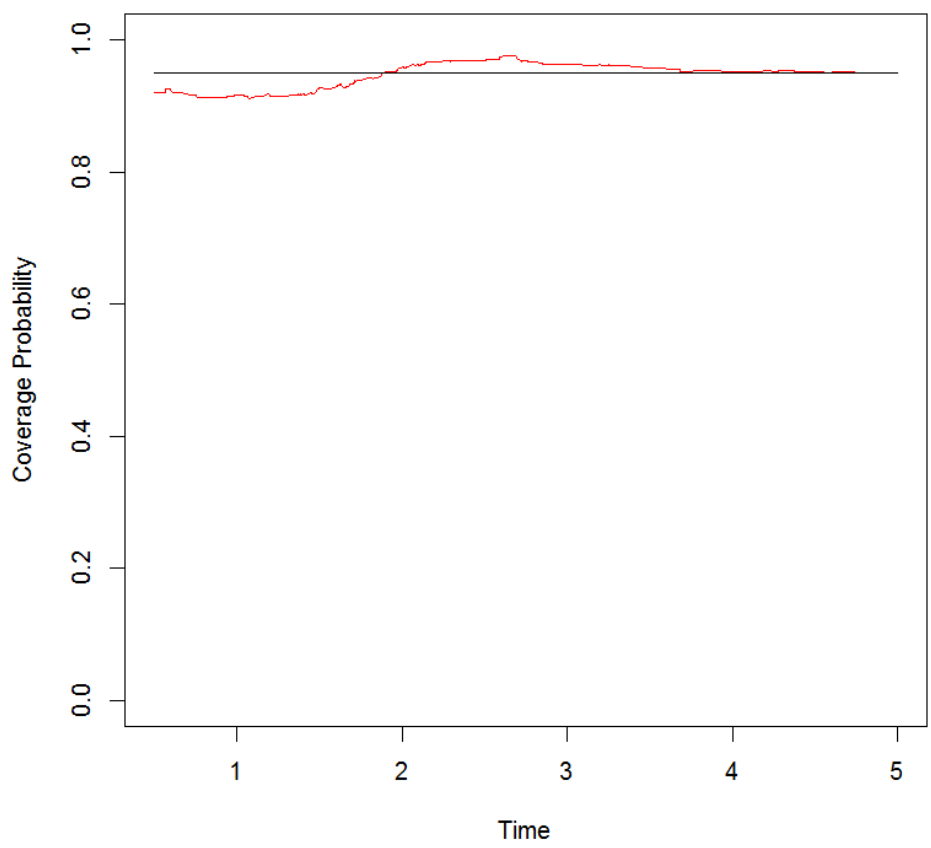

Figure A.12: Coverage probabilities for the Weibull function of Case 2 
A.3 Case 3: $n=500$ and number of time panels $=10$

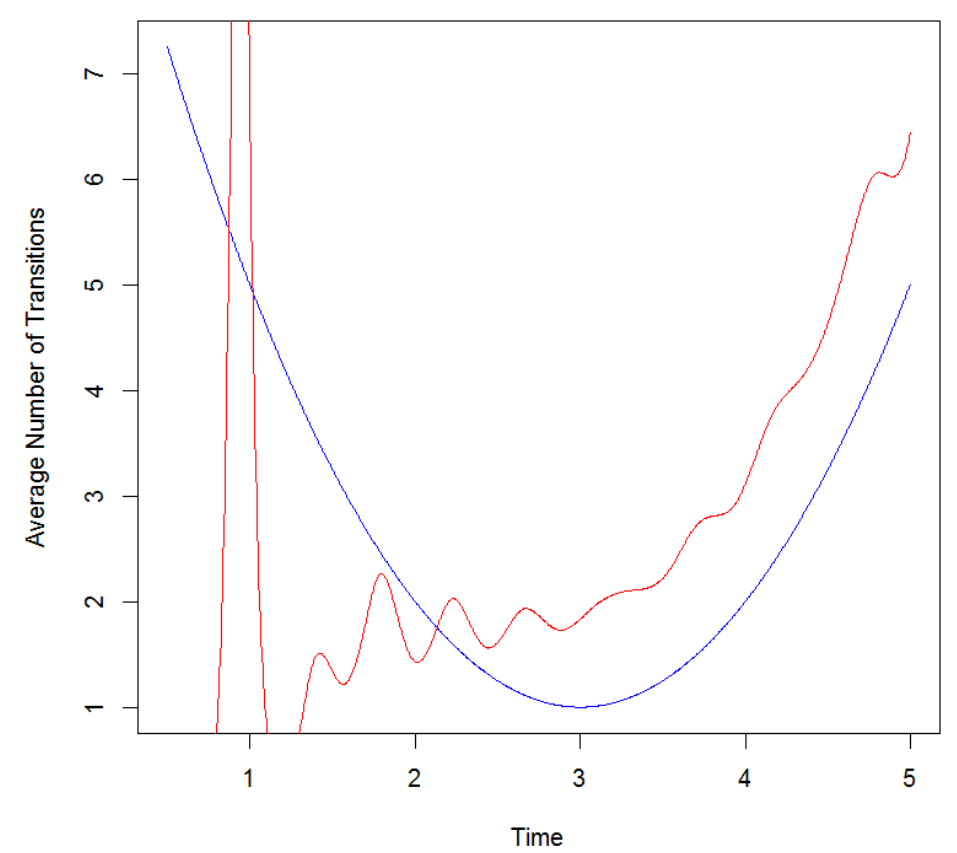

Figure A.13: Quadratic fit for Case 3 with $\lambda=27$ 


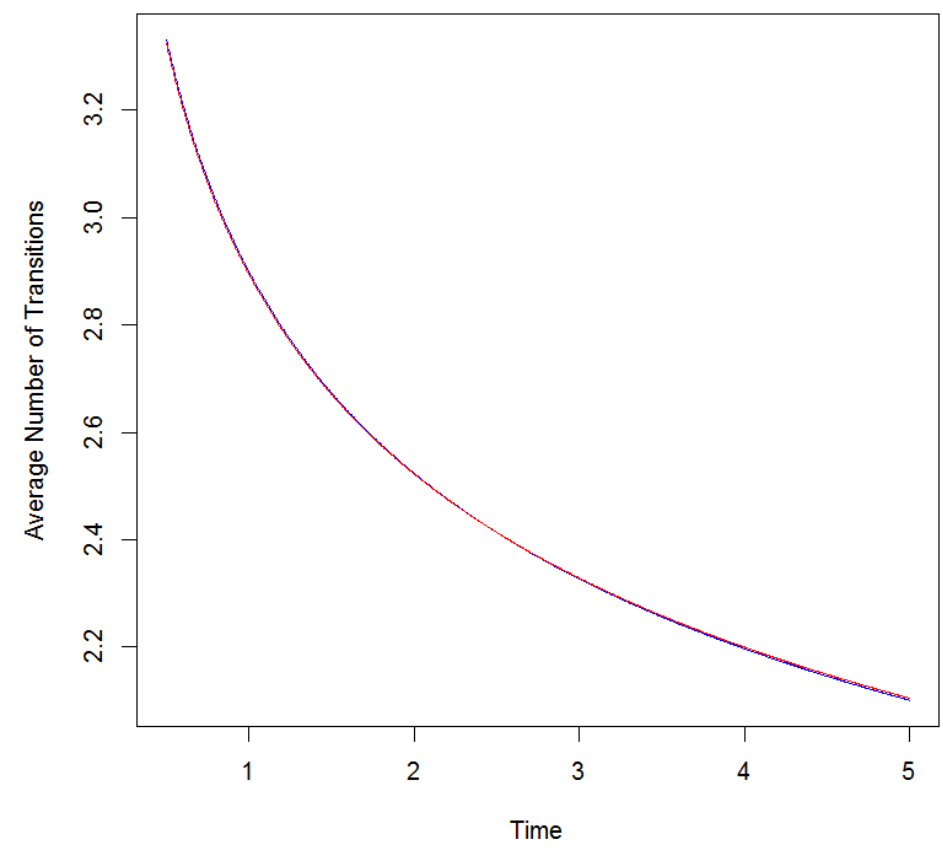

Figure A.14: Weibull fit for Case 3 with $\lambda=10000$

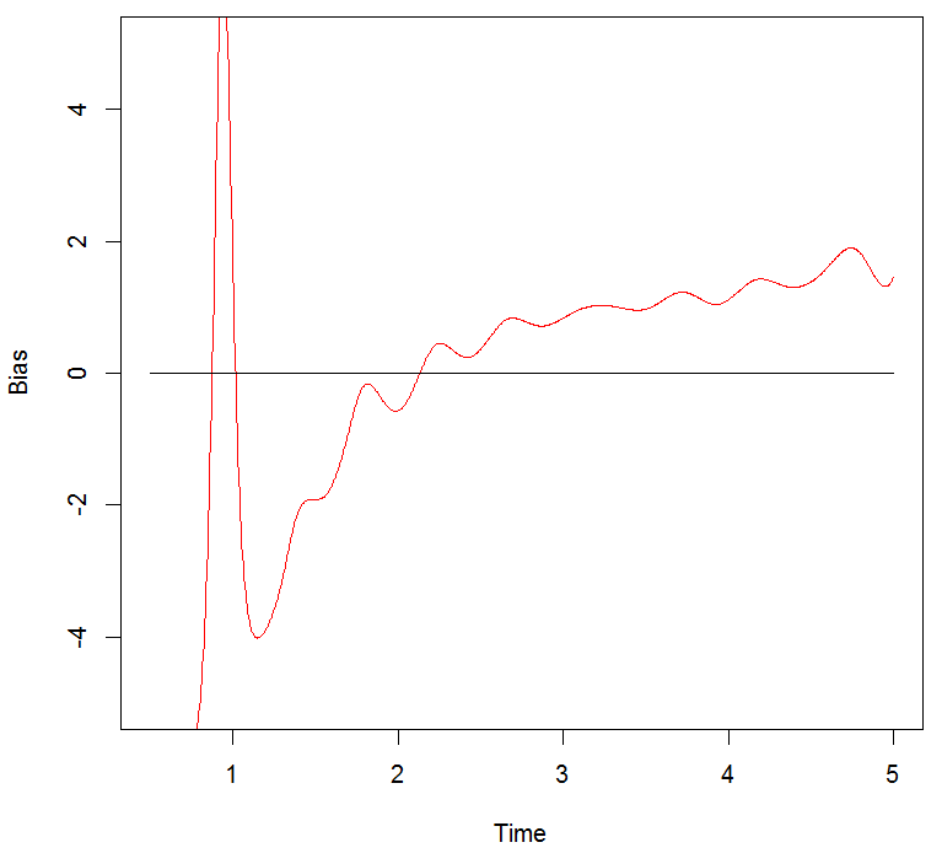

Figure A.15: Bias for the quadratic function of Case 3 


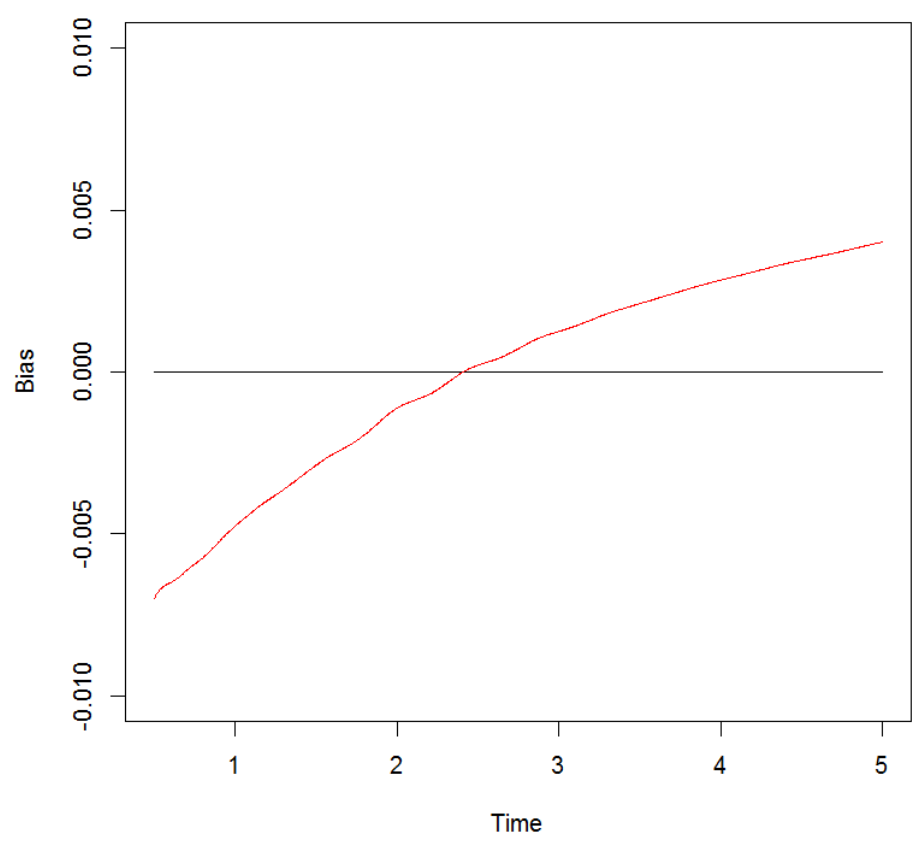

Figure A.16: Bias for the Weibull function of Case 3. We note that the graph is zoomed in significantly.

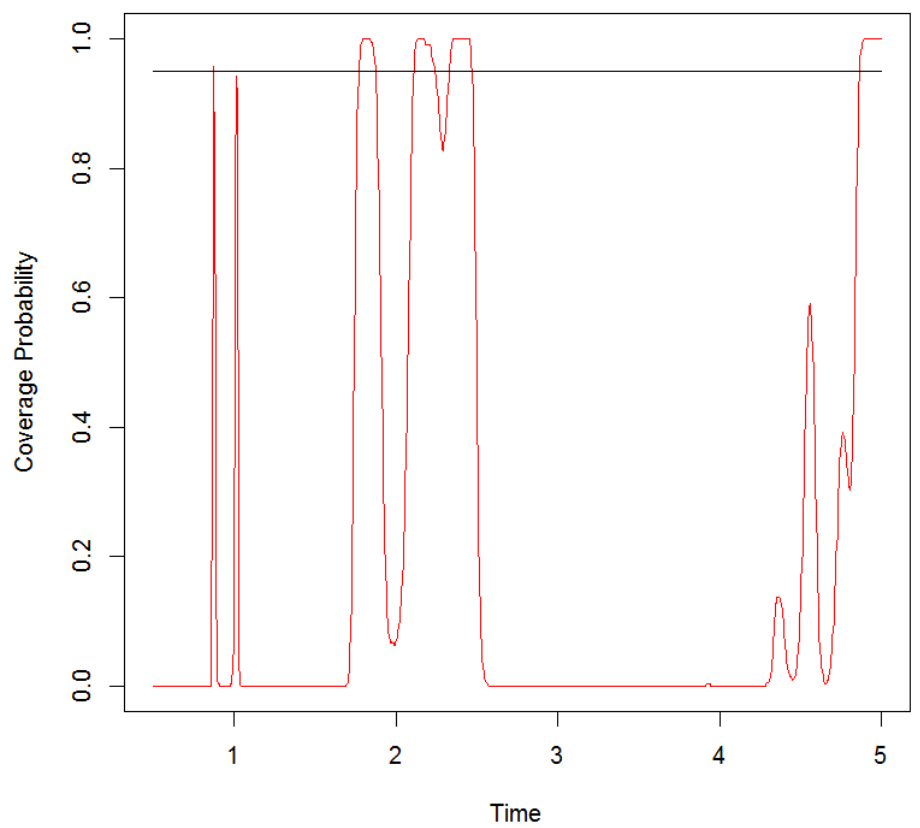

Figure A.17: Coverage probabilities for the quadratic function of Case 3 


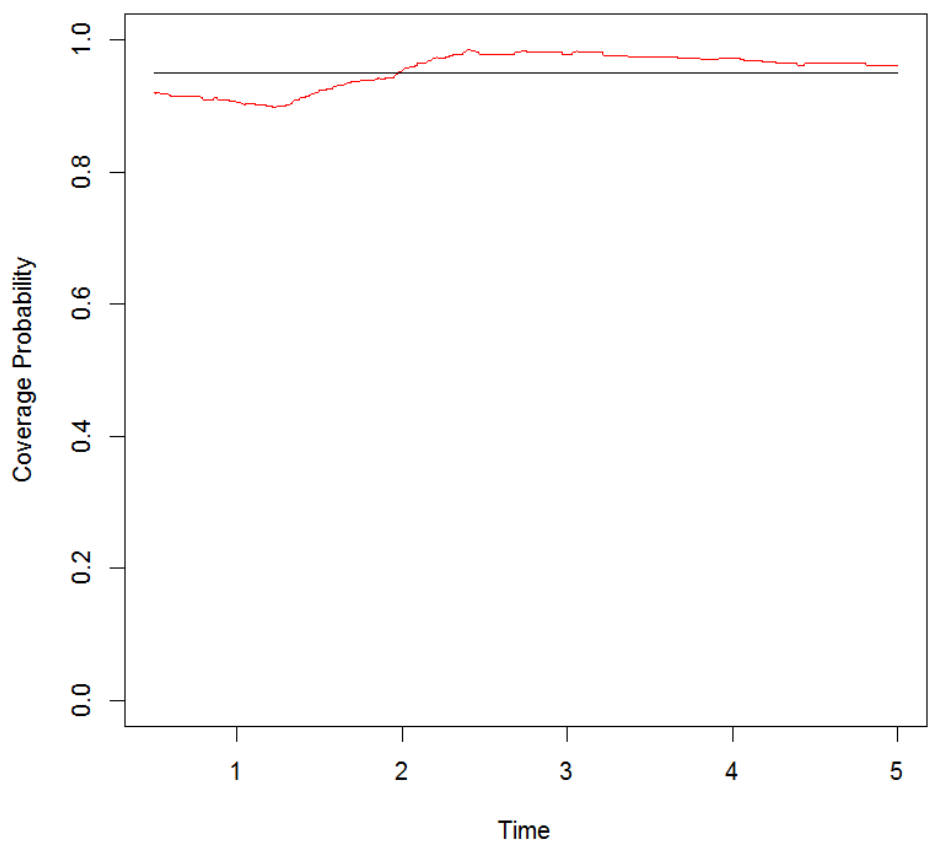

Figure A.18: Coverage probabilities for the Weibull function of Case 3 
A.4 Case 4: $n=250$ and number of time panels $=50$

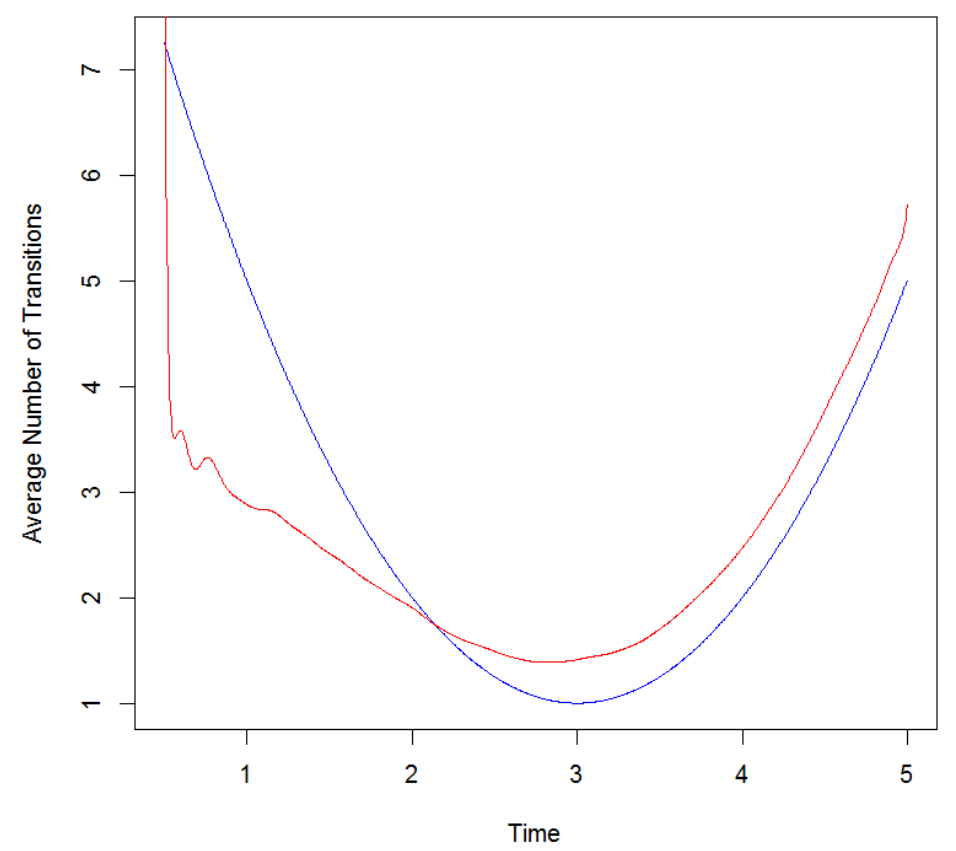

Figure A.19: Quadratic fit for Case 4 with $\lambda=8$ 


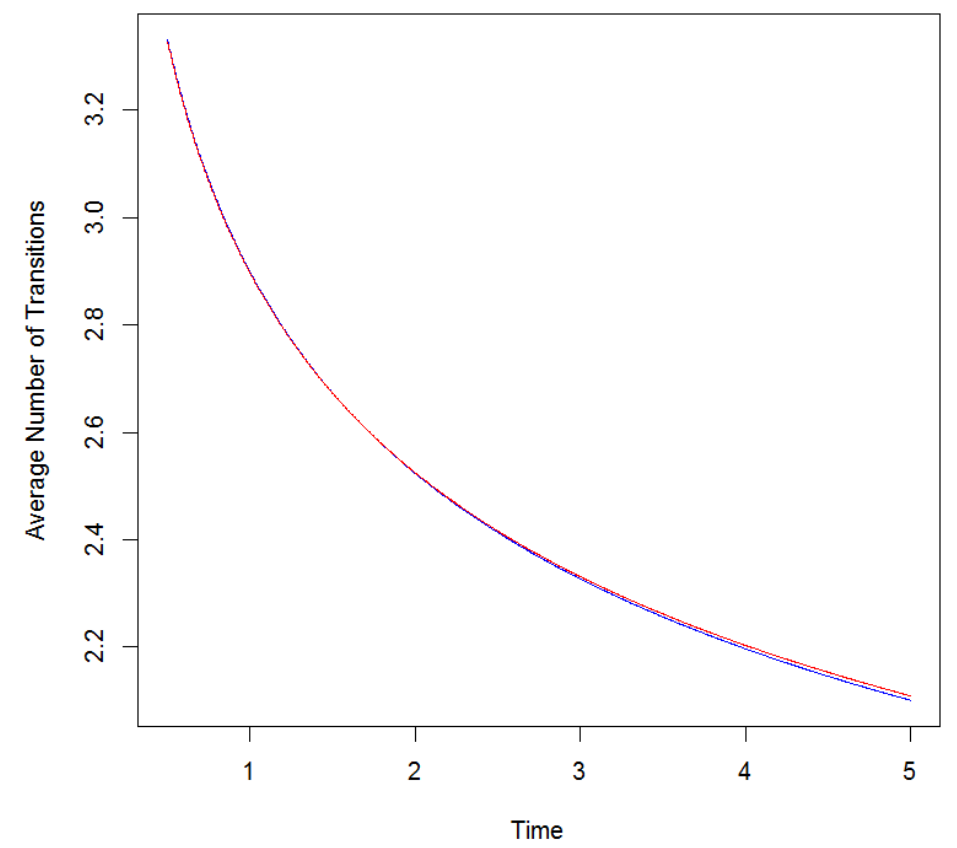

Figure A.20: Weibull fit for Case 4 with $\lambda=10000$

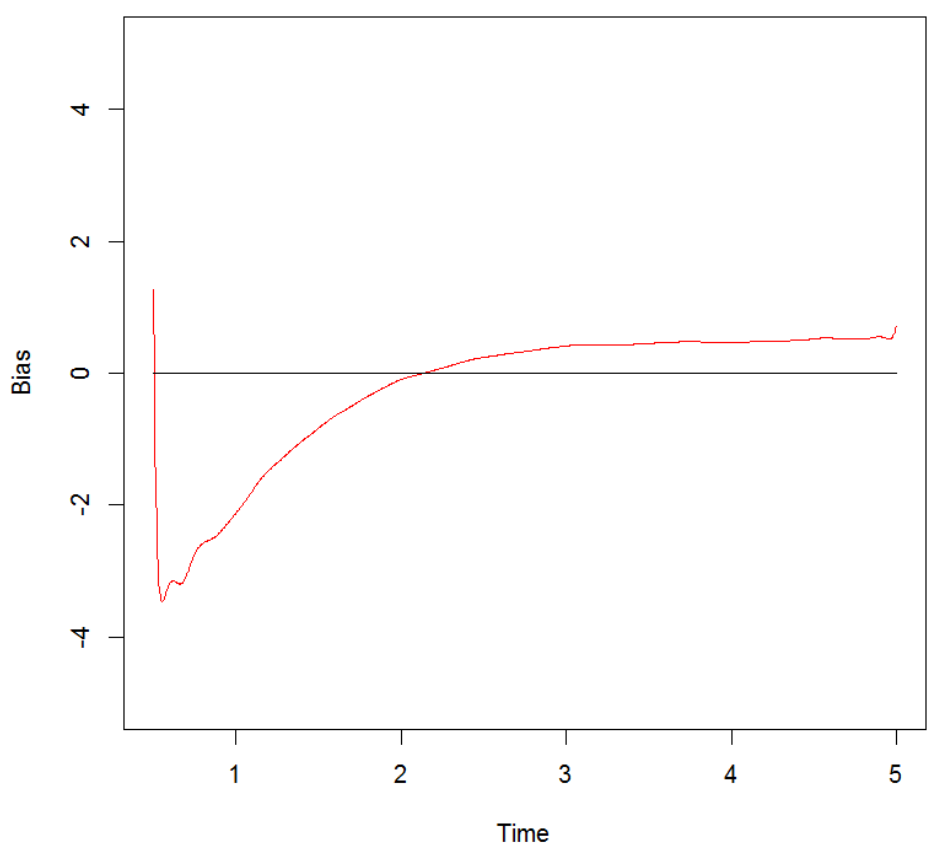

Figure A.21: Bias for the quadratic function of Case 4 


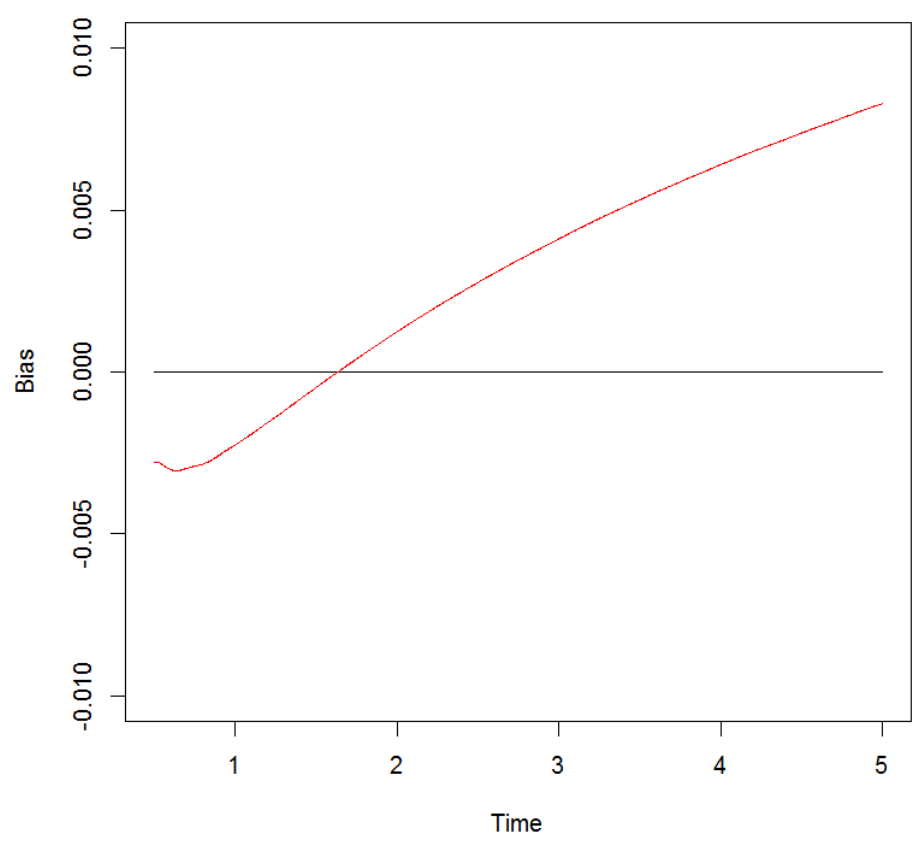

Figure A.22: Bias for the Weibull function of Case 4. We note that the graph is zoomed in significantly.

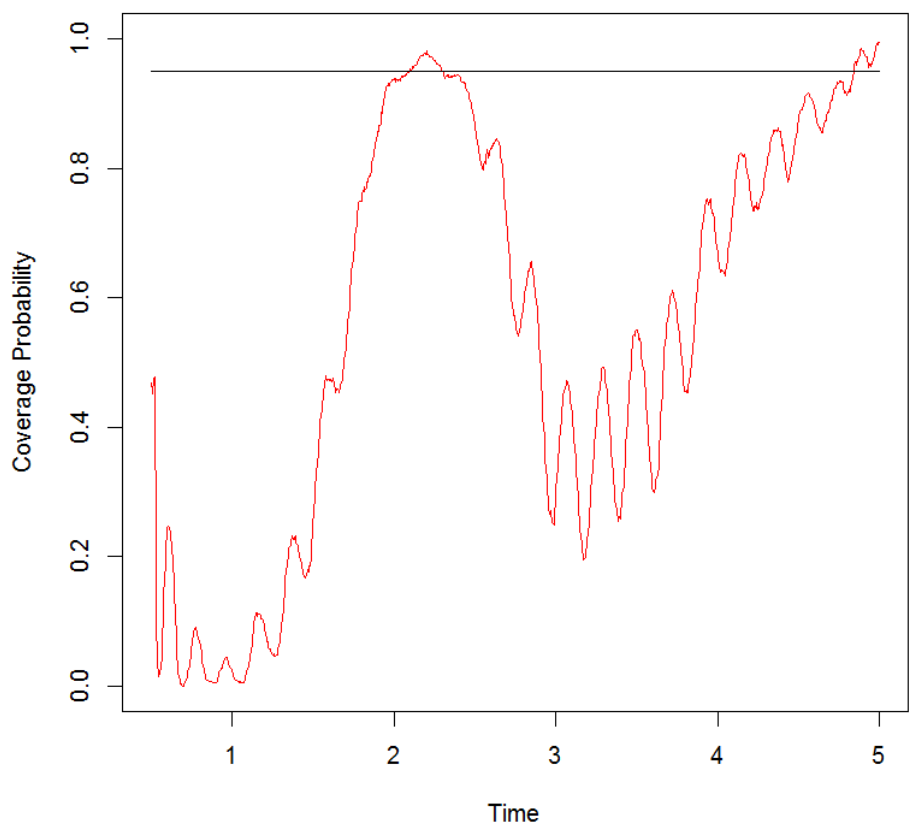

Figure A.23: Coverage probabilities for the quadratic function of Case 4 


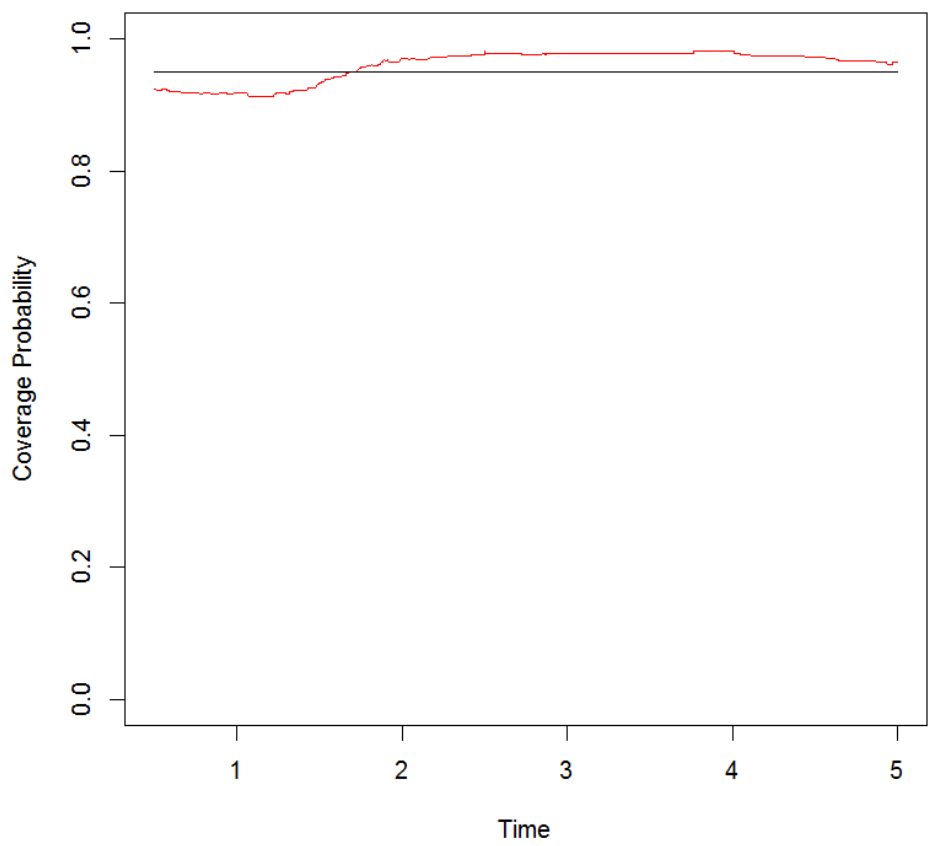

Figure A.24: Coverage probabilities for the Weibull function of Case 4 
A.5 Case 5: $n=250$ and number of time panels $=25$

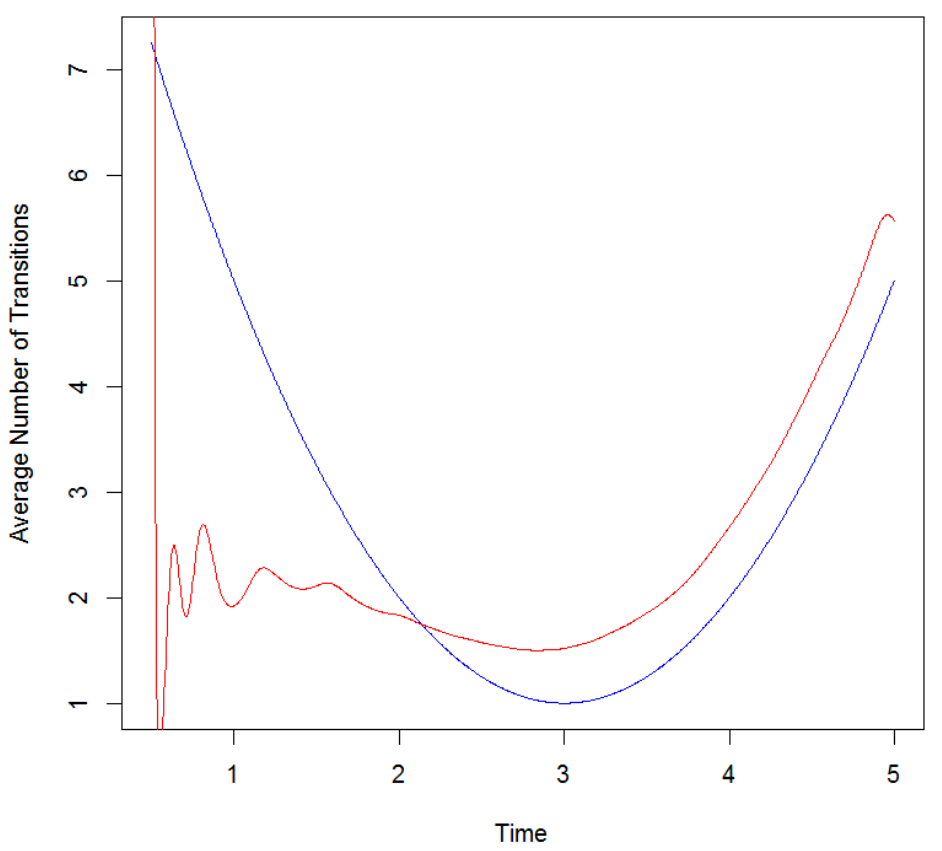

Figure A.25: Quadratic fit for Case 5 with $\lambda=10$ 


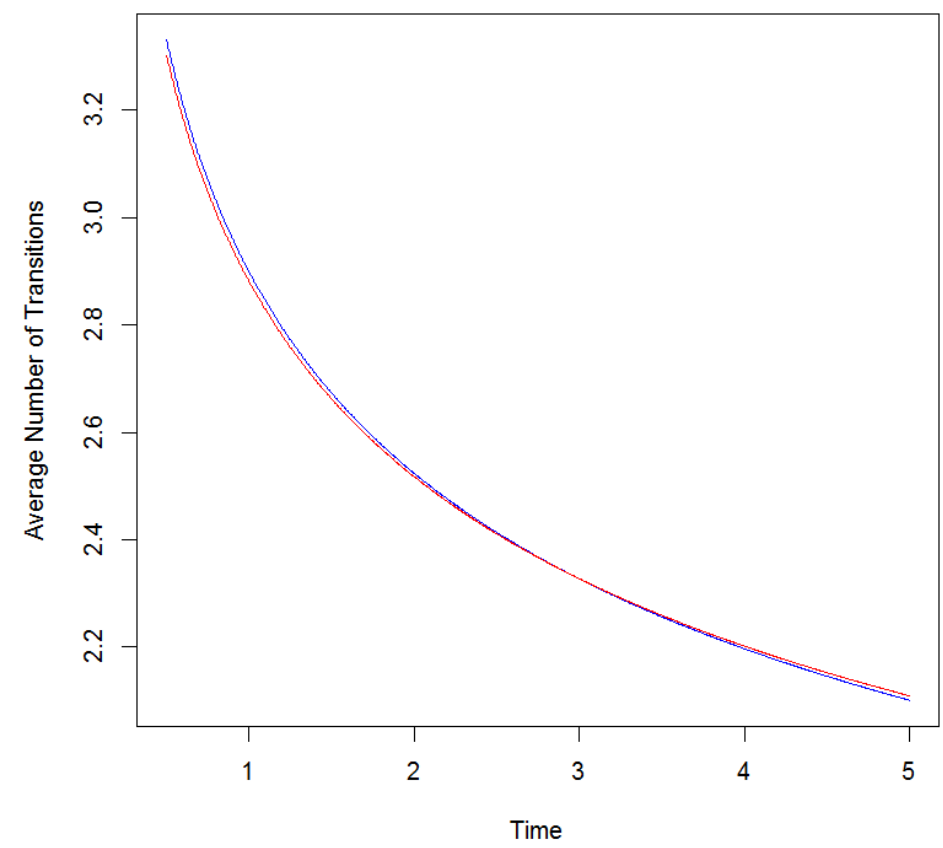

Figure A.26: Weibull fit for Case 5 with $\lambda=10000$

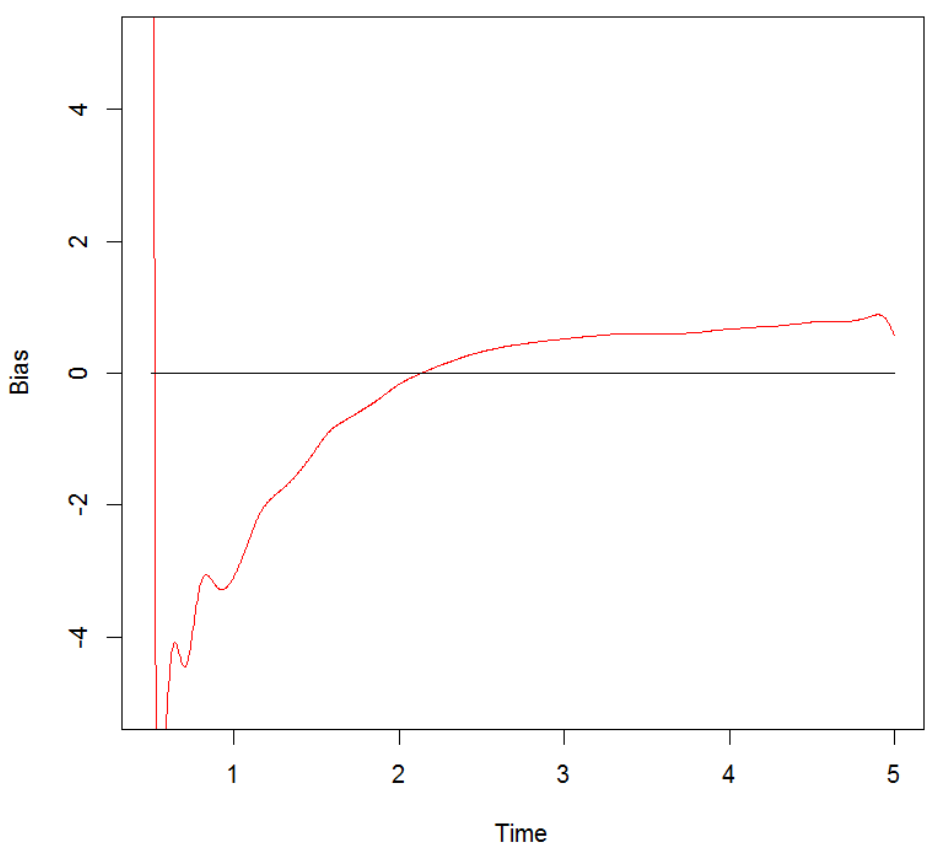

Figure A.27: Bias for the quadratic function of Case 5 


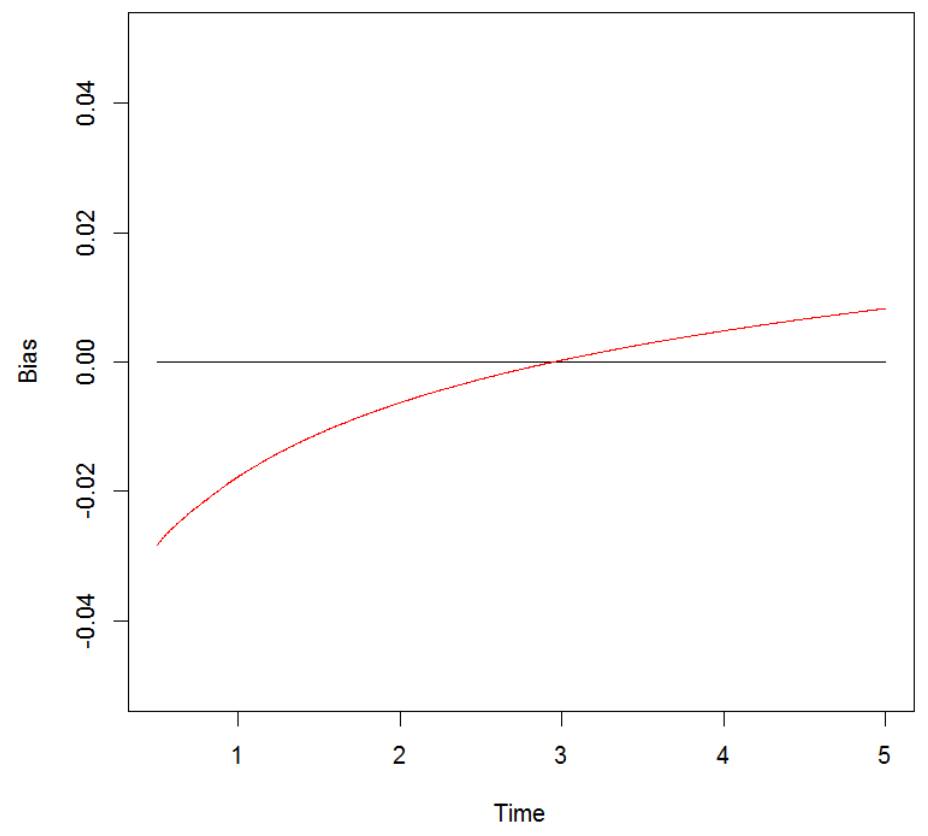

Figure A.28: Bias for the Weibull function of Case 5. We note that the graph is zoomed in significantly.

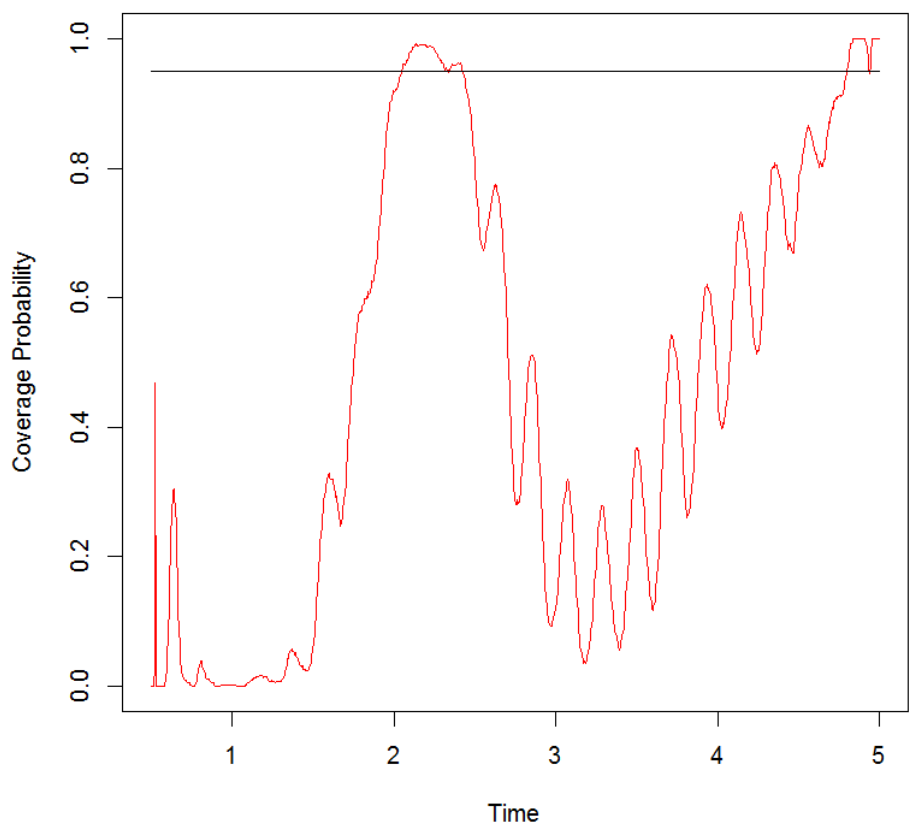

Figure A.29: Coverage probabilities for the quadratic function of Case 5 


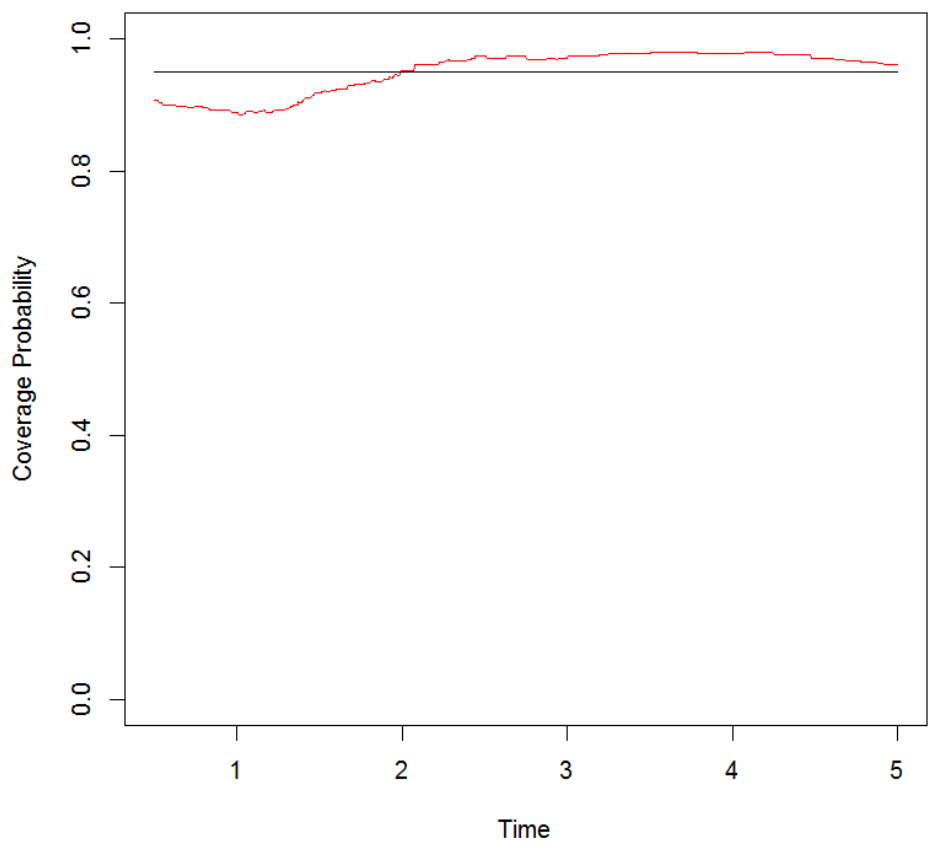

Figure A.30: Coverage probabilities for the Weibull function of Case 5 
A.6 Case 6: $n=250$ and number of time panels $=10$

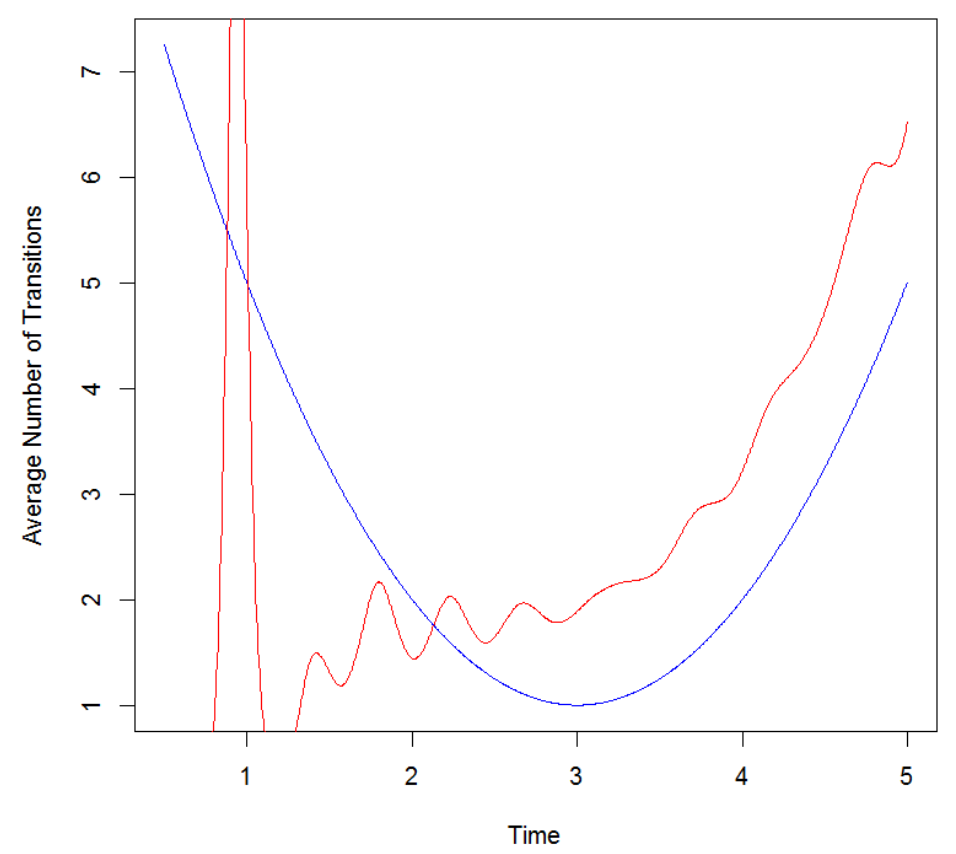

Figure A.31: Quadratic fit for Case 6 with $\lambda=15$ 


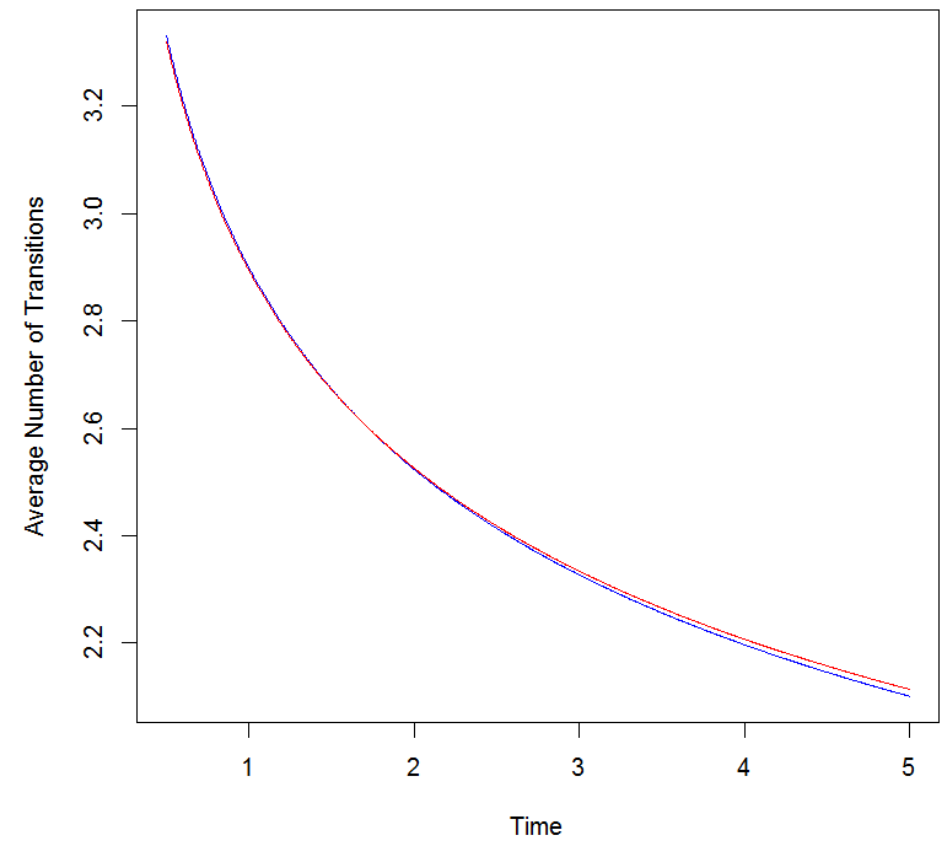

Figure A.32: Weibull fit for Case 6 with $\lambda=10000$

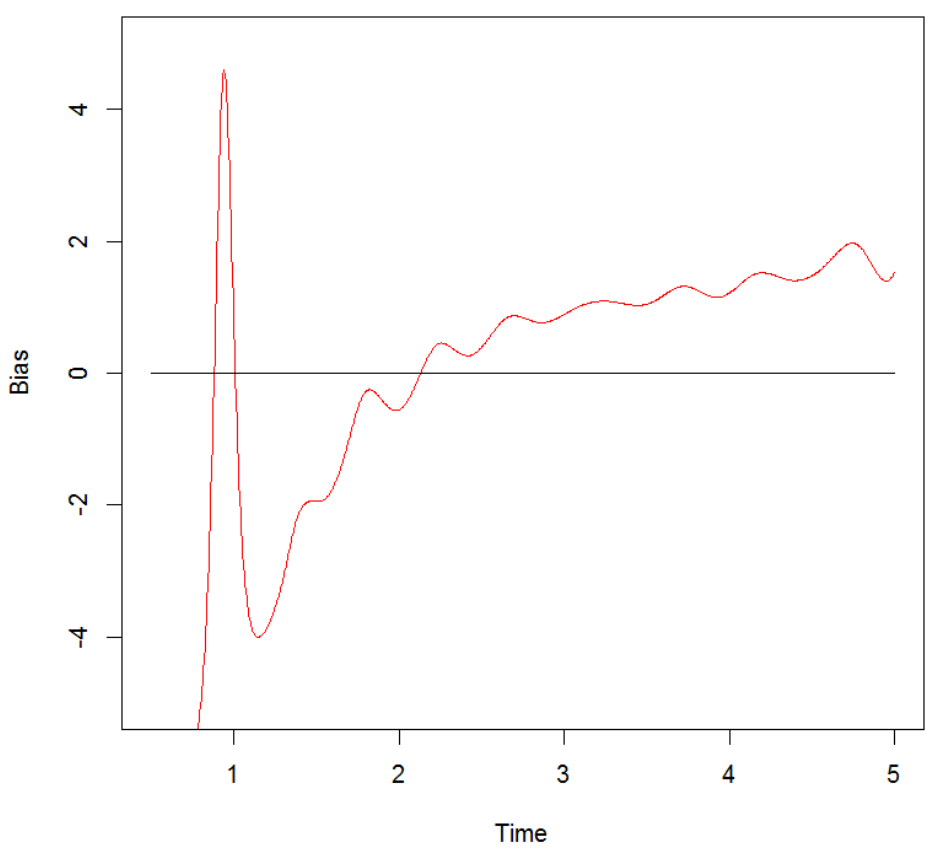

Figure A.33: Bias for the quadratic function of Case 6 


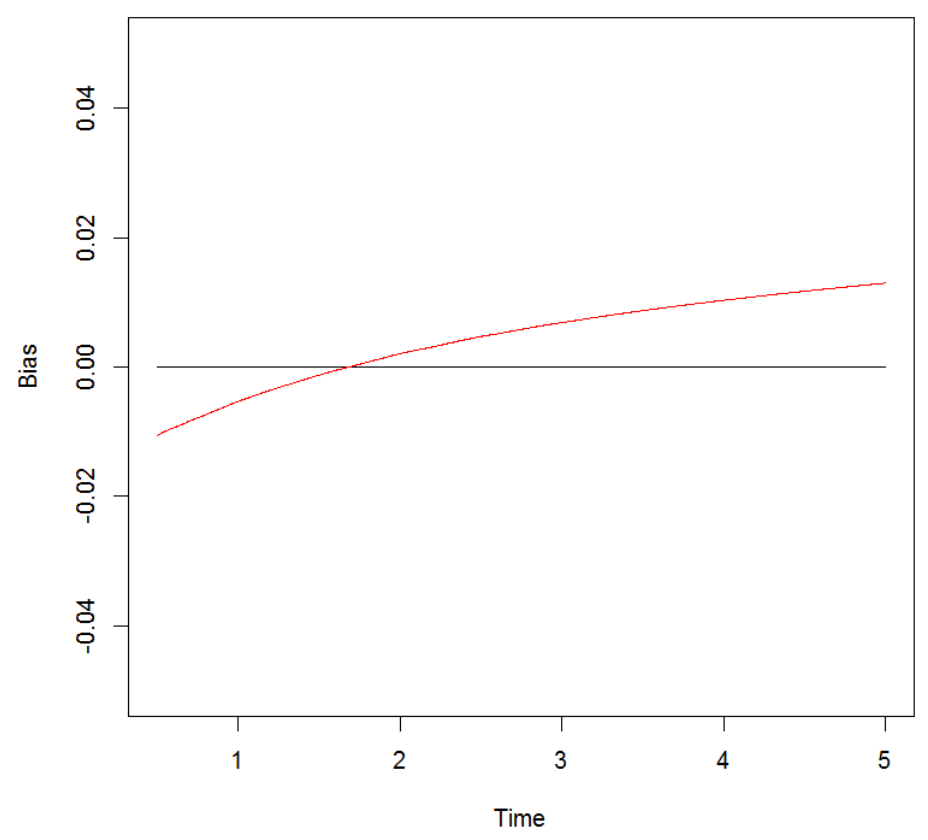

Figure A.34: Bias for the Weibull function of Case 6. We note that the graph is zoomed in significantly.

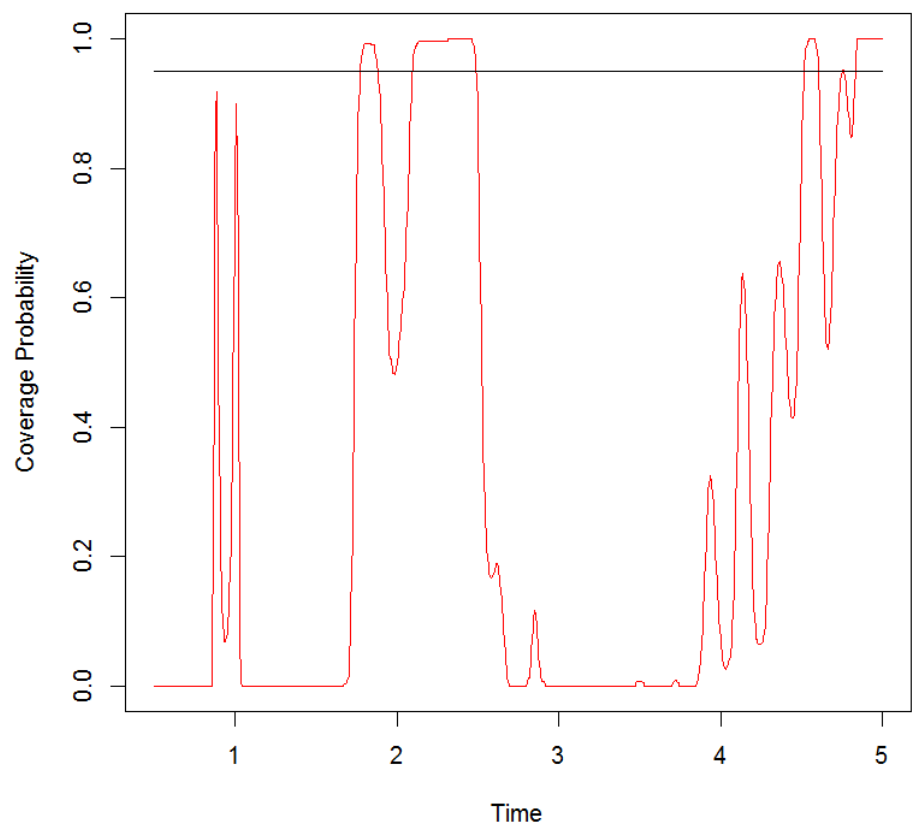

Figure A.35: Coverage probabilities for the quadratic function of Case 6 


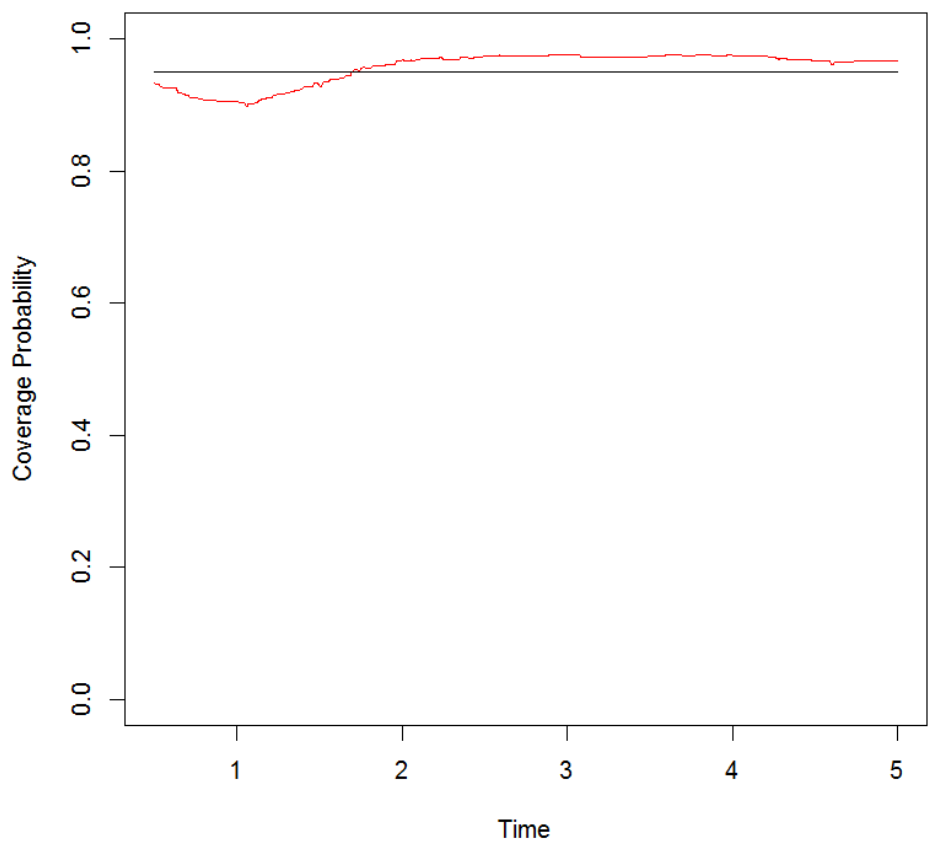

Figure A.36: Coverage probabilities for the Weibull function of Case 6 
A.7 Case 7: $n=150$ and number of time panels $=50$

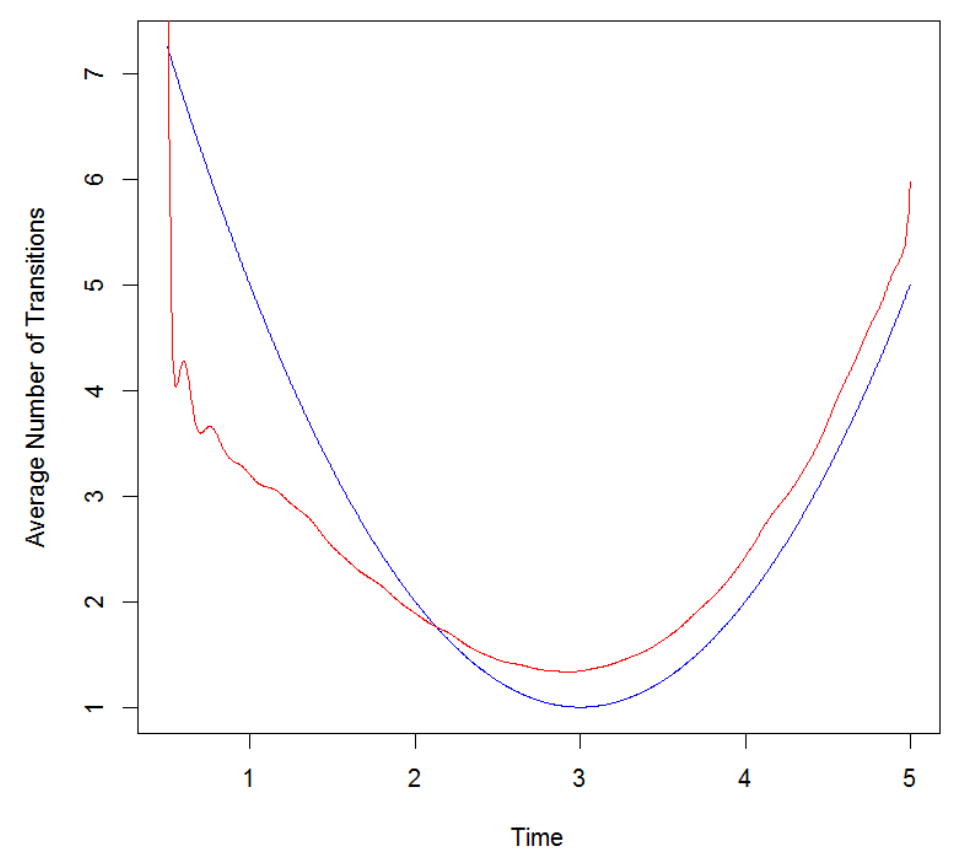

Figure A.37: Quadratic fit for Case 7 with $\lambda=4$ 


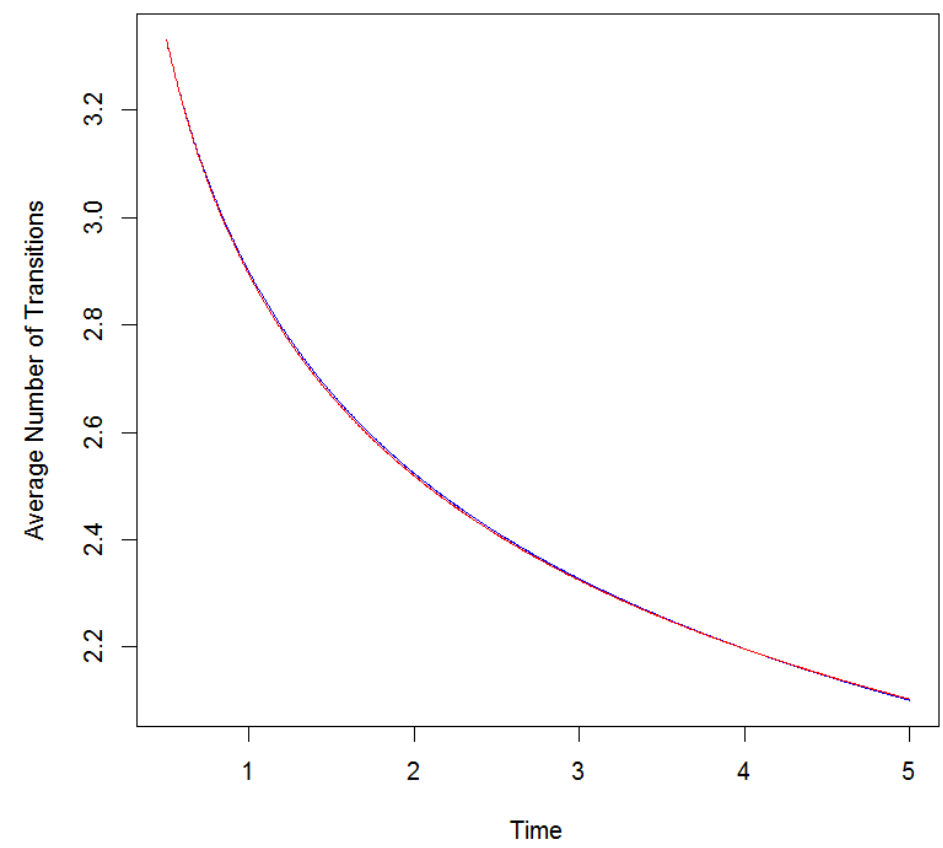

Figure A.38: Weibull fit for Case 7 with $\lambda=10000$

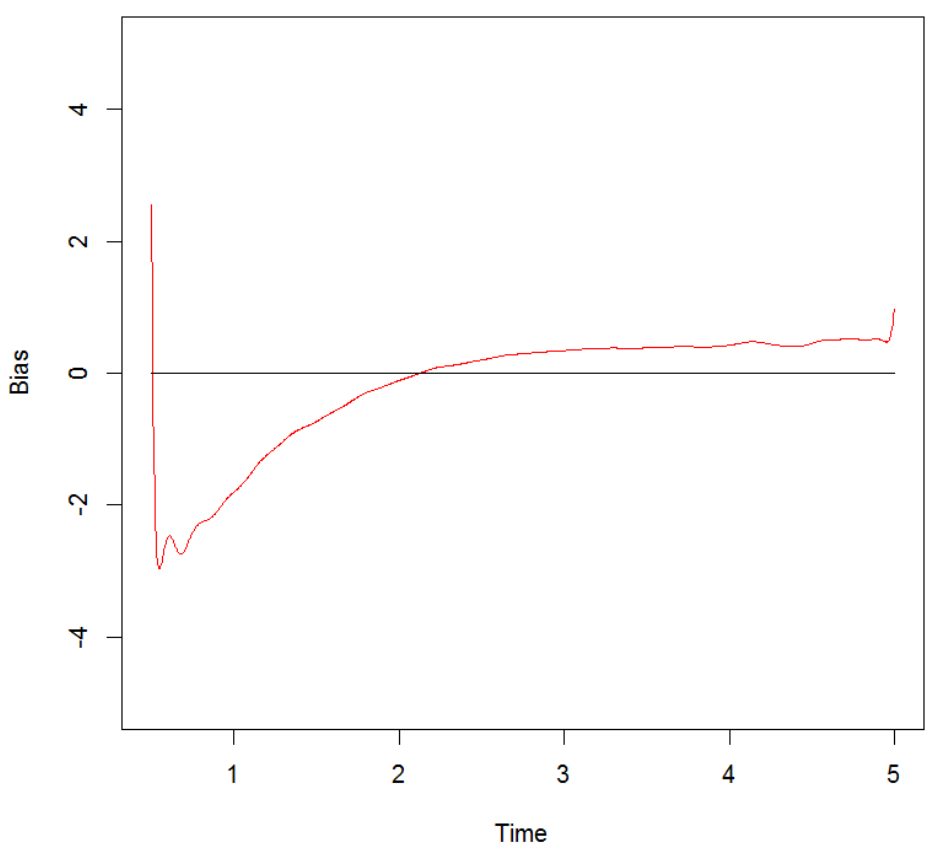

Figure A.39: Bias for the quadratic function of Case 7 


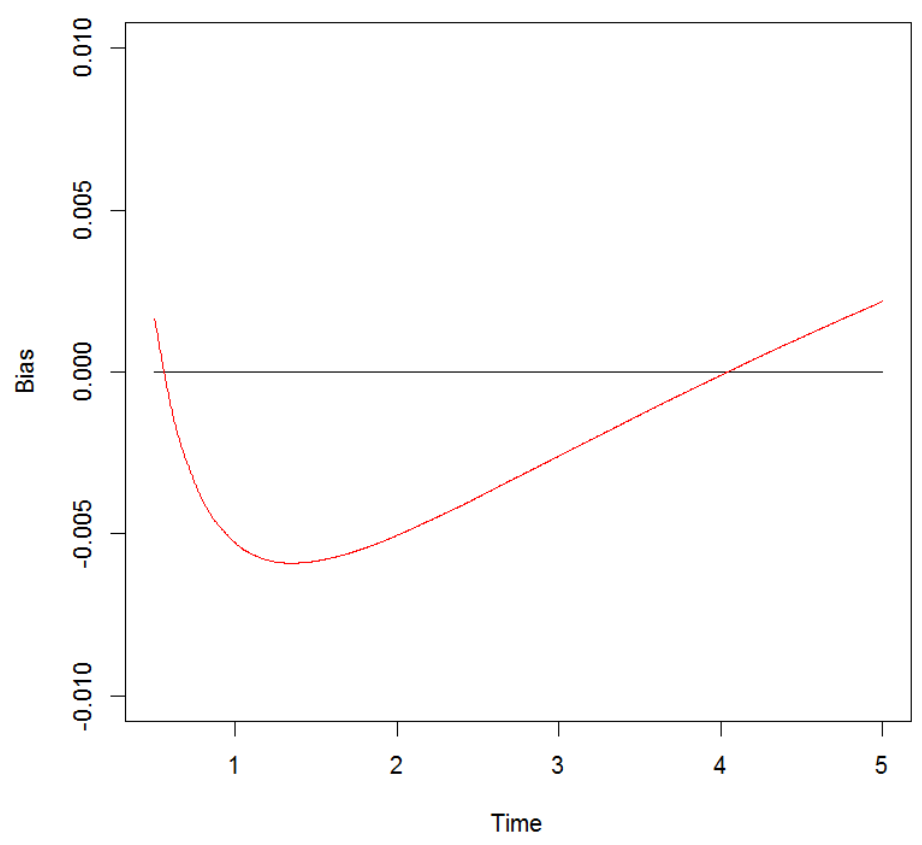

Figure A.40: Bias for the Weibull function of Case 7. We note that the graph is zoomed in significantly.

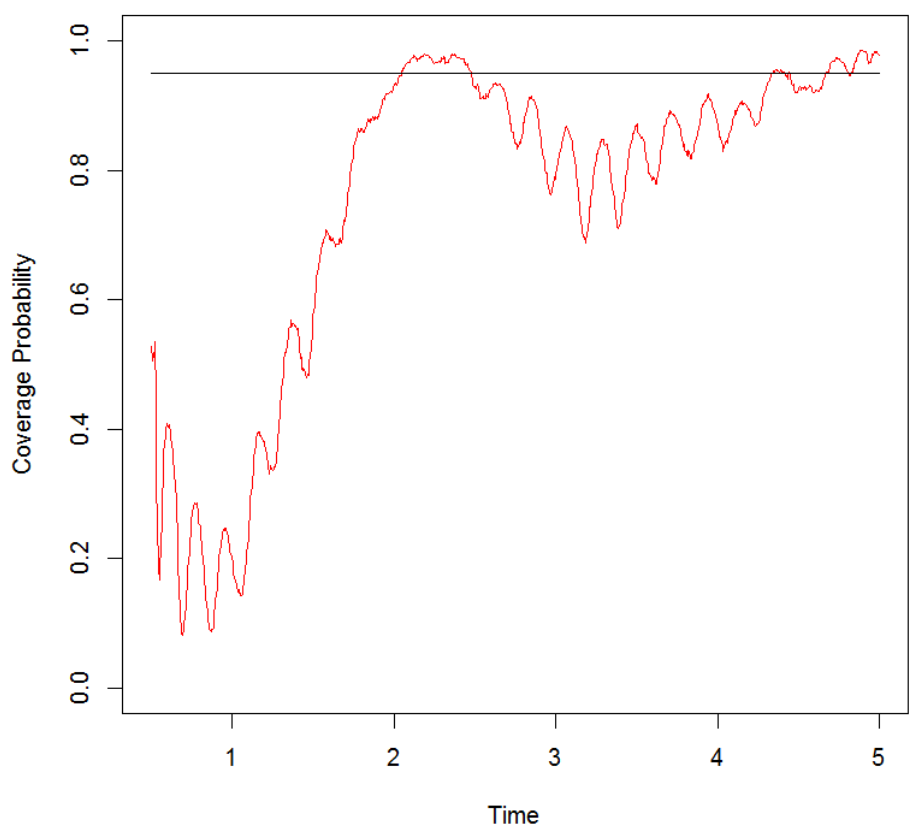

Figure A.41: Coverage probabilities for quadratic function of Case 7 


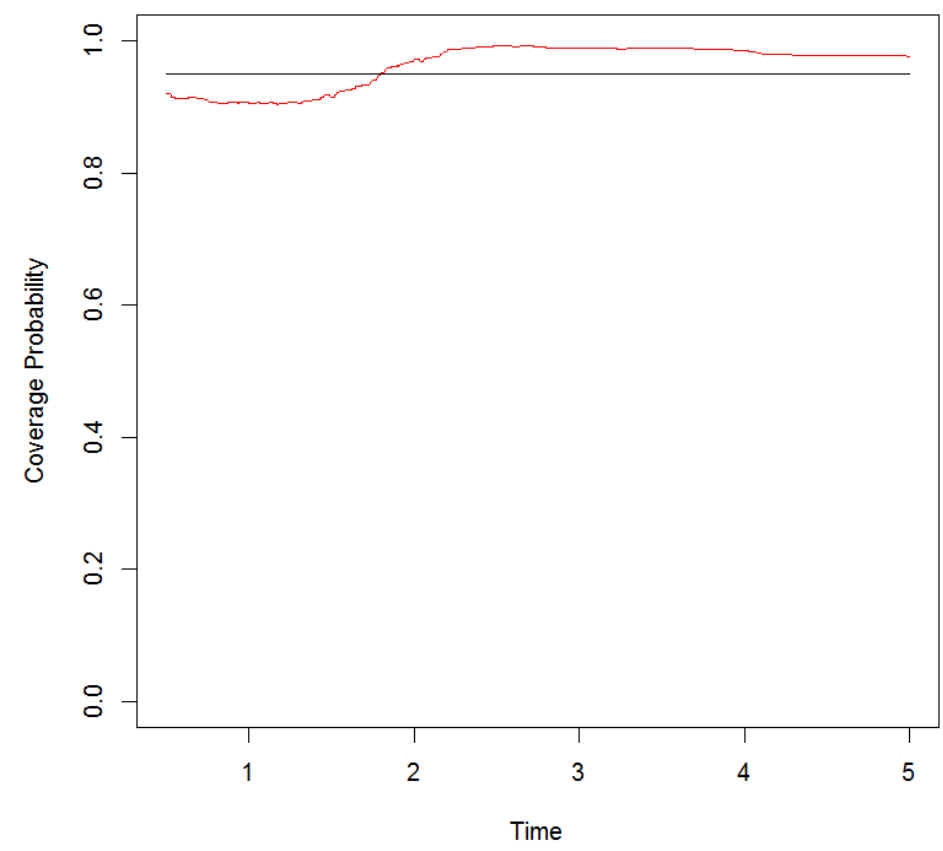

Figure A.42: Coverage probabilities for the Weibull function of Case 7 
A.8 Case 8: $n=150$ and number of time panels $=25$

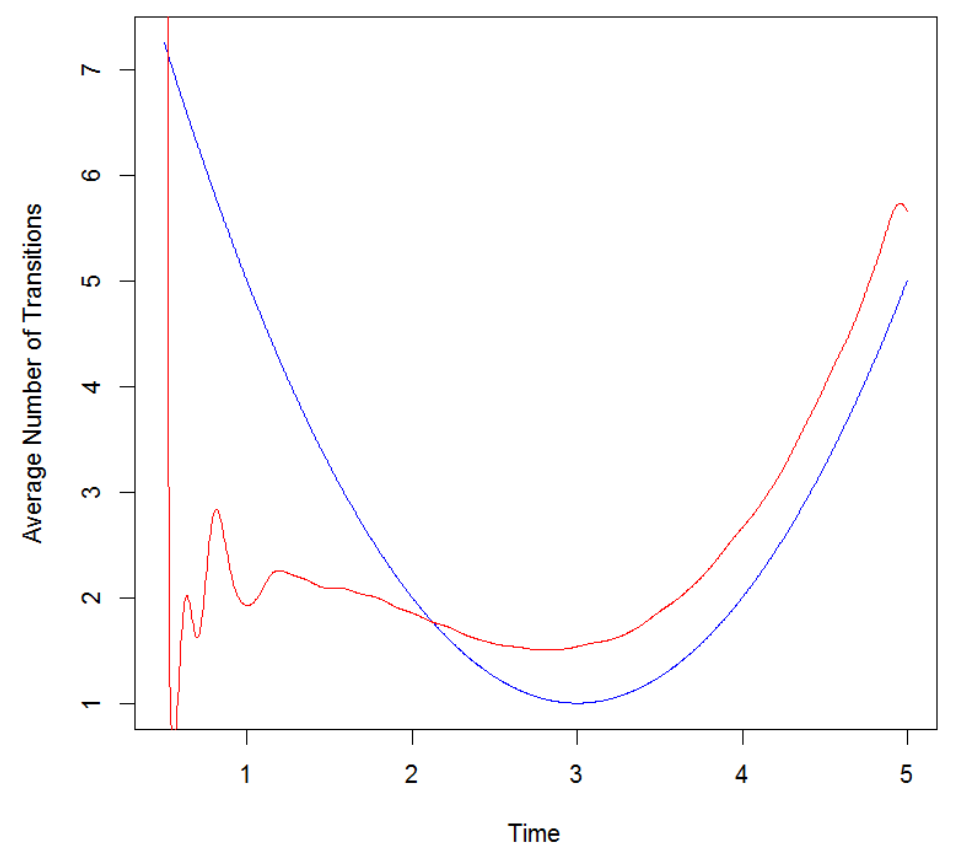

Figure A.43: Quadratic fit for Case 8 with $\lambda=6$ 


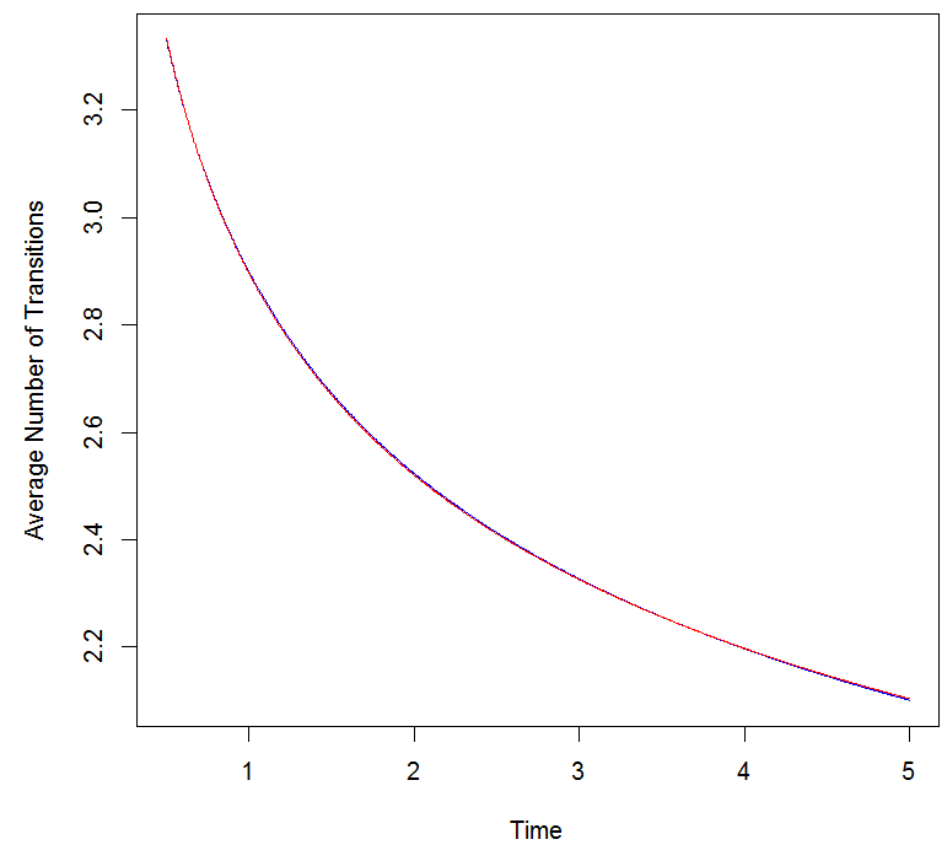

Figure A.44: Weibull fit for Case 8 with $\lambda=10000$

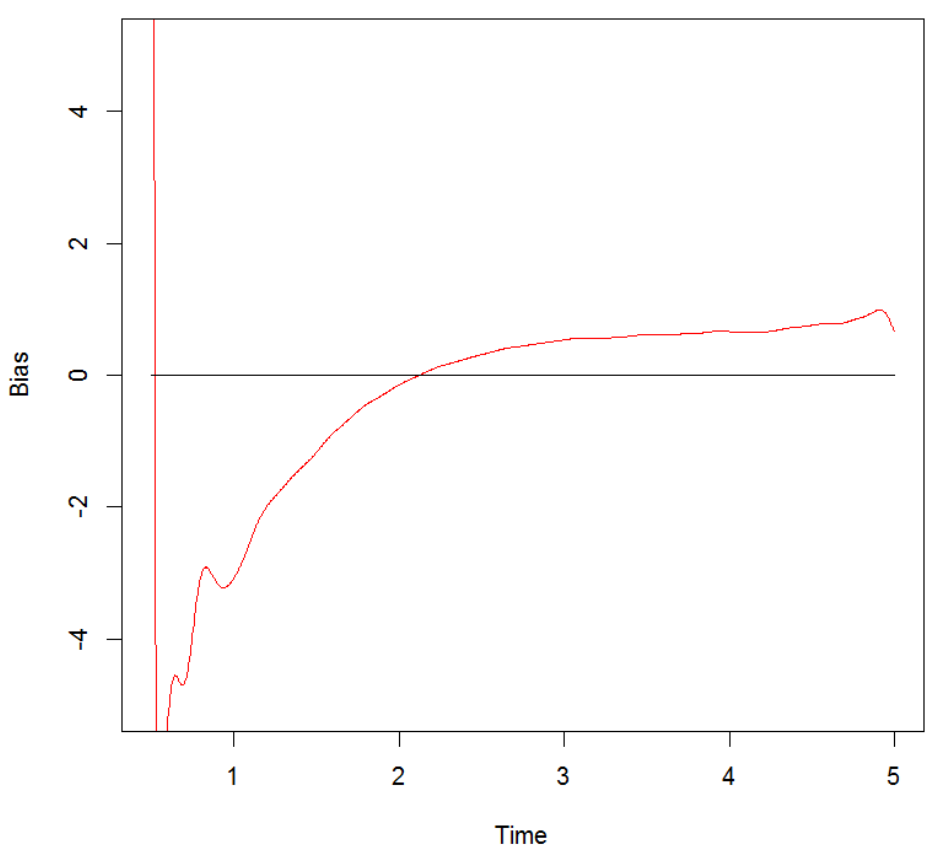

Figure A.45: Bias for the quadratic function of Case 8 


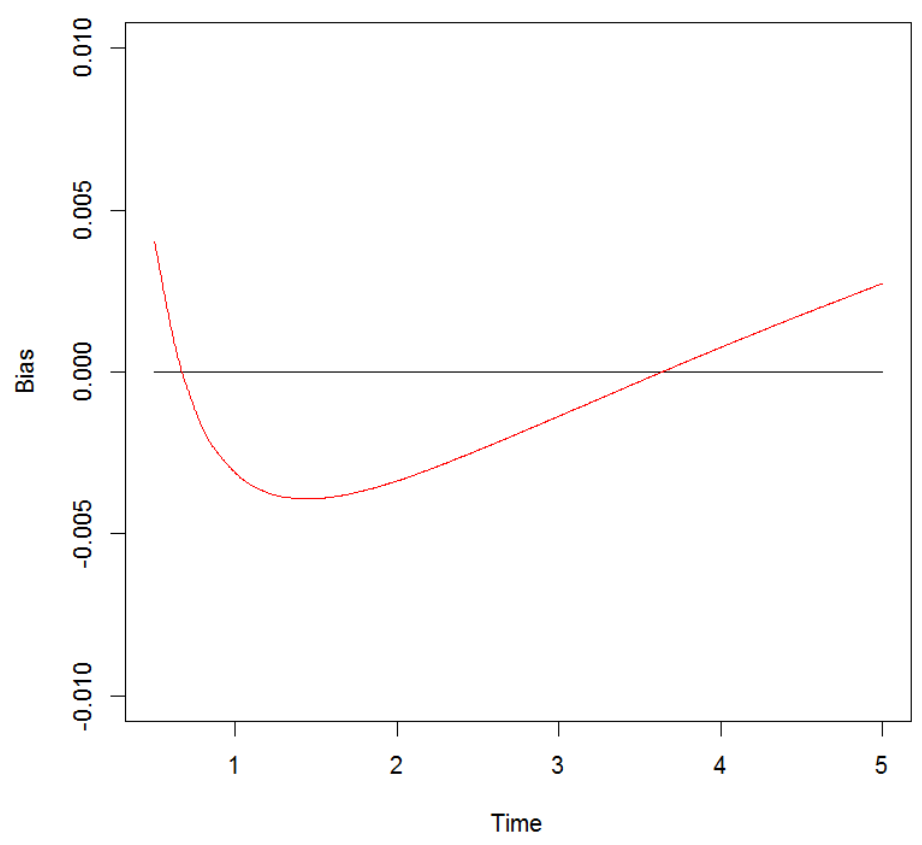

Figure A.46: Bias for the Weibull function of Case 8. We note that the graph is zoomed in significantly.

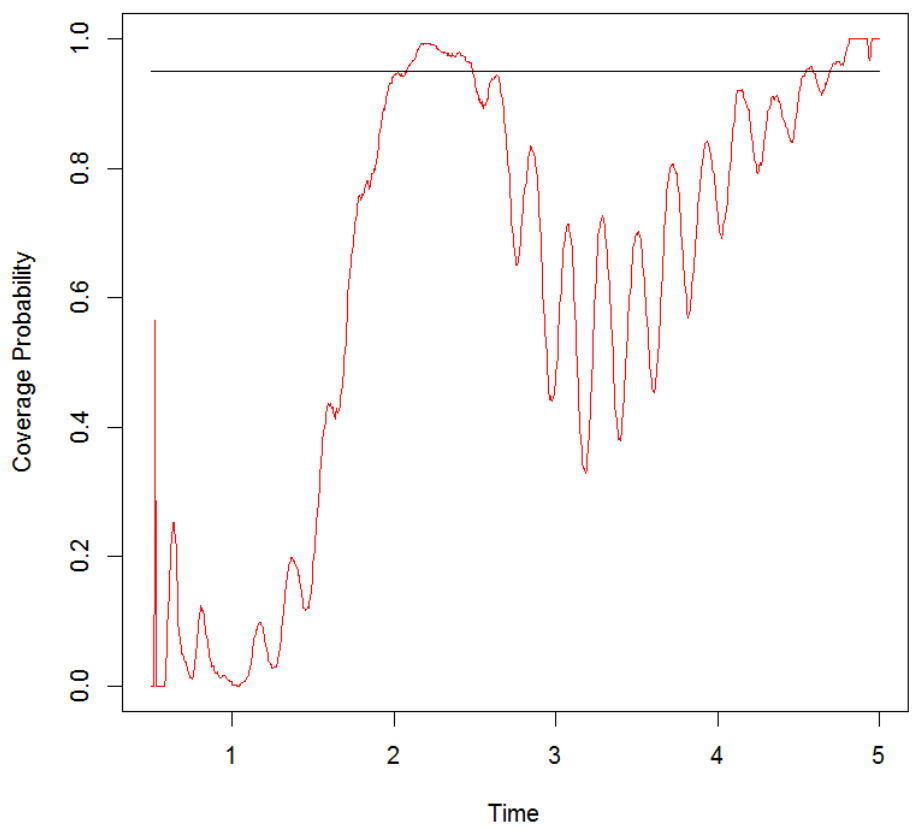

Figure A.47: Coverage probabilities for the quadratic function of Case 8 


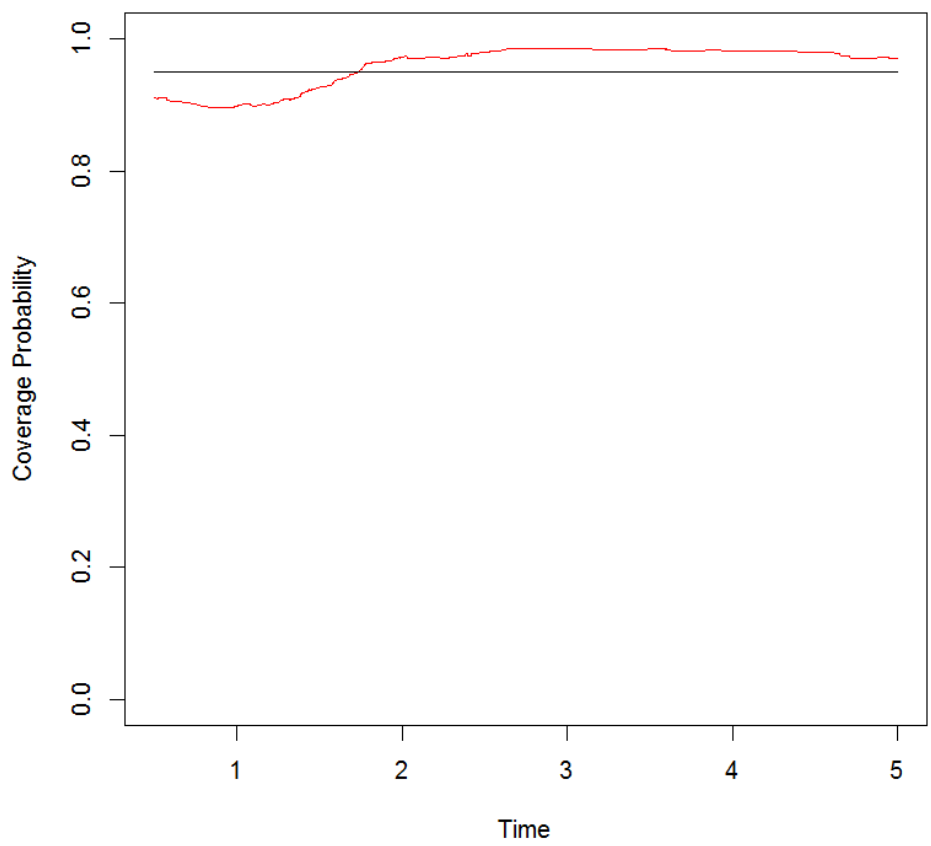

Figure A.48: Coverage probabilities for the Weibull function of Case 8 
A.9 Case 9: $n=150$ and number of time panels $=10$

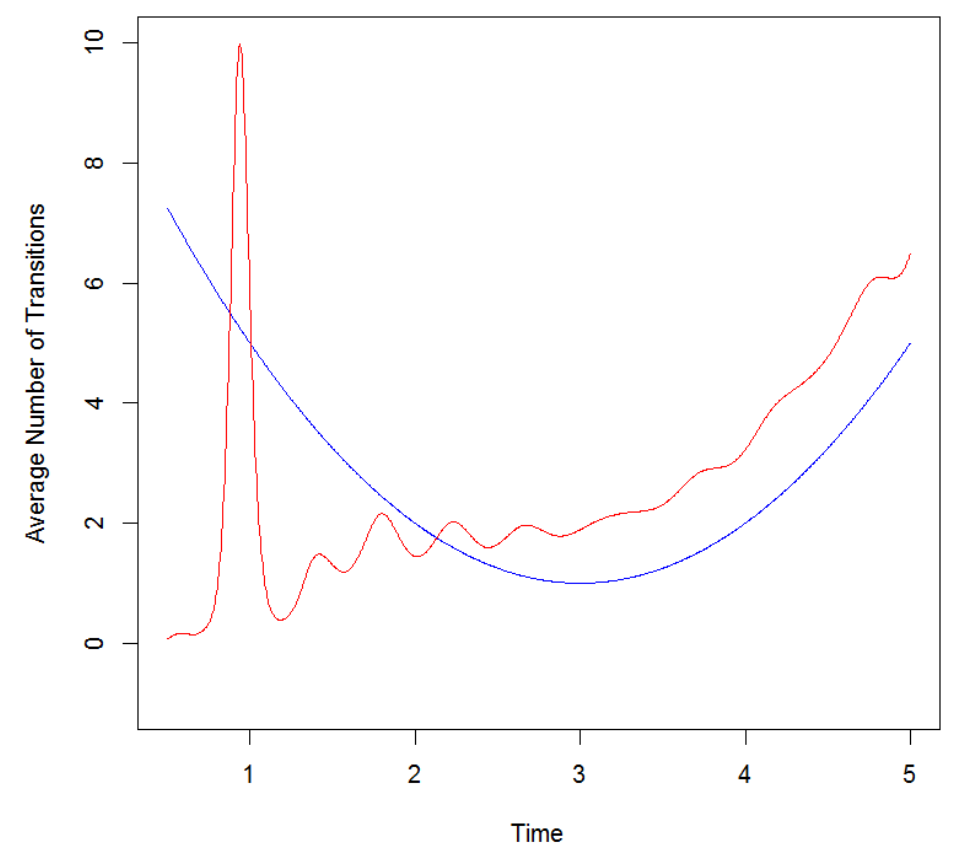

Figure A.49: Quadratic fit for Case 9 with $\lambda=9$

\section{A.10 Case 10: Quadratic Function with a Divided Difference Penalty}

These are the full set of graphs for the quadratic function from the case where the quadratic function used a divided difference penalty instead of the model based Weibull penalty. 


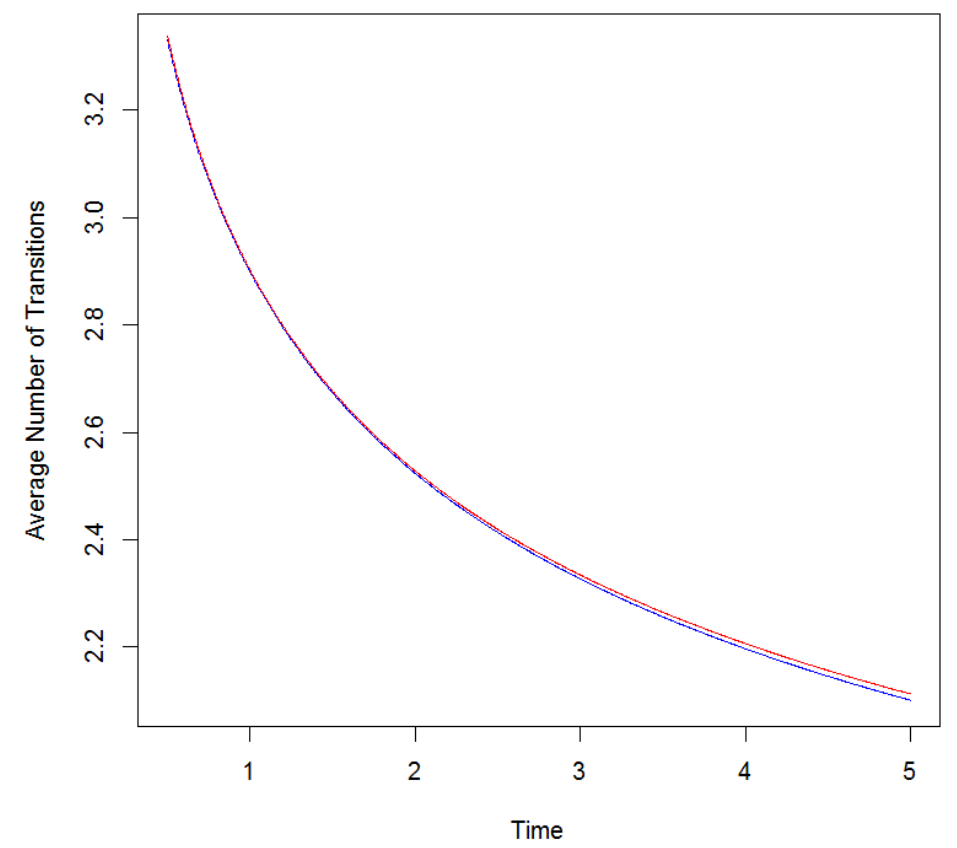

Figure A.50: Weibull fit for Case 9 with $\lambda=10000$

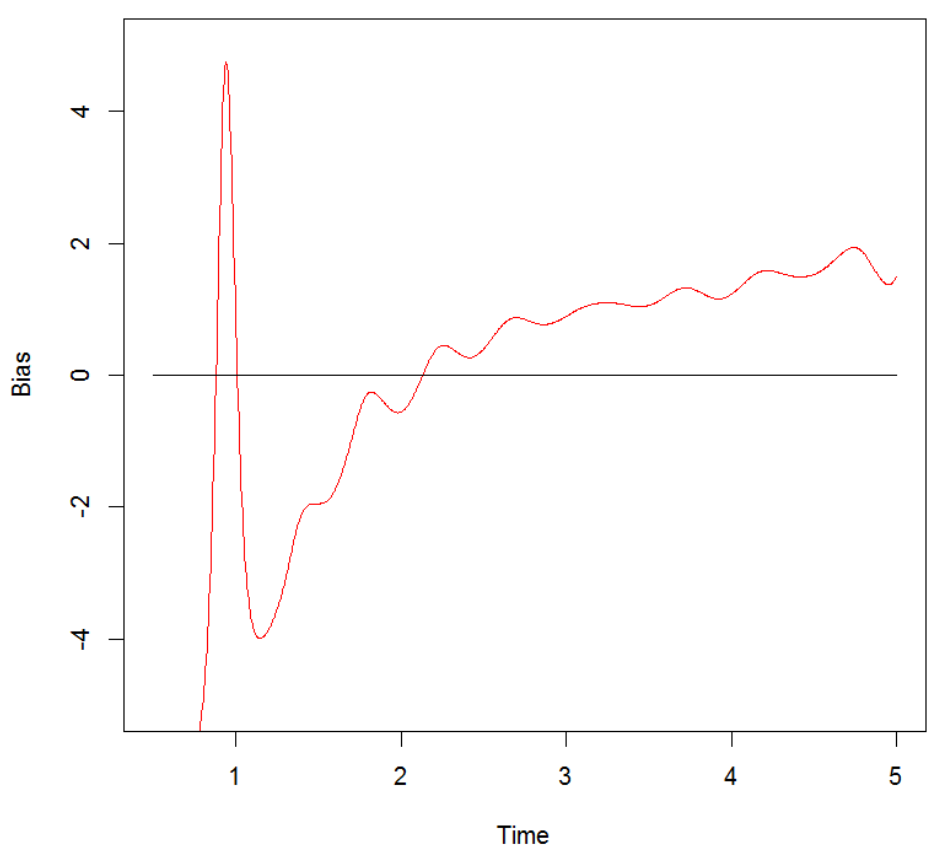

Figure A.51: Bias for the quadratic function of Case 9 


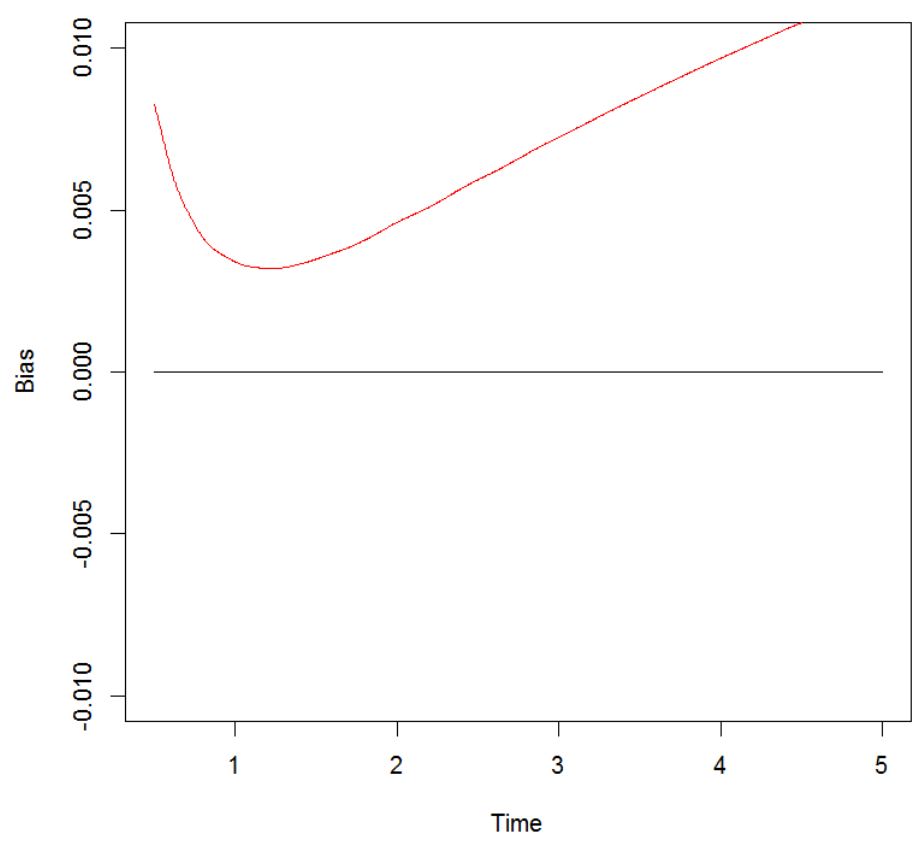

Figure A.52: Bias for the Weibull function of Case 9. We note that the graph is zoomed in significantly.

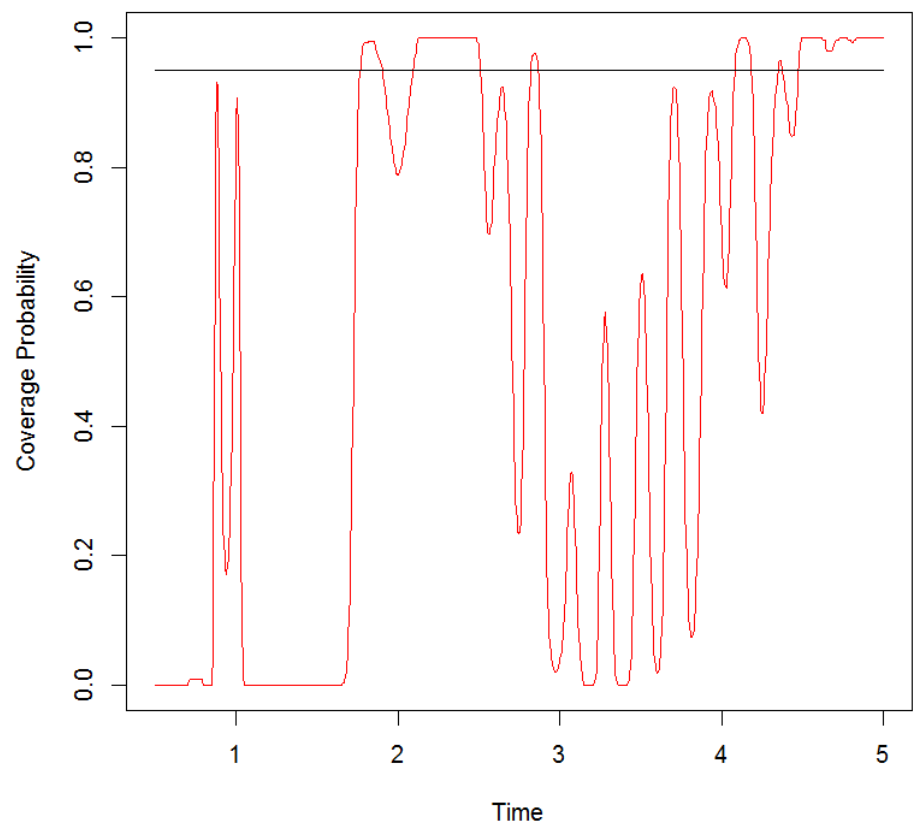

Figure A.53: Coverage probabilities for the quadratic function of Case 9 


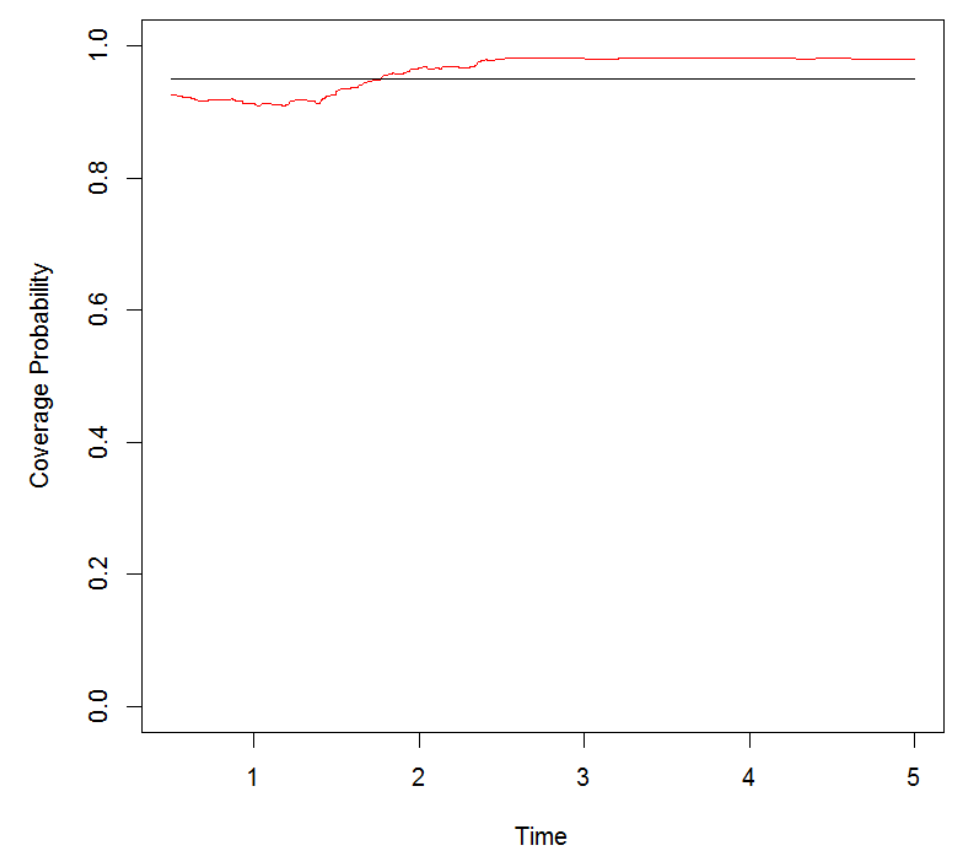

Figure A.54: Coverage probabilities for the Weibull function of Case 9

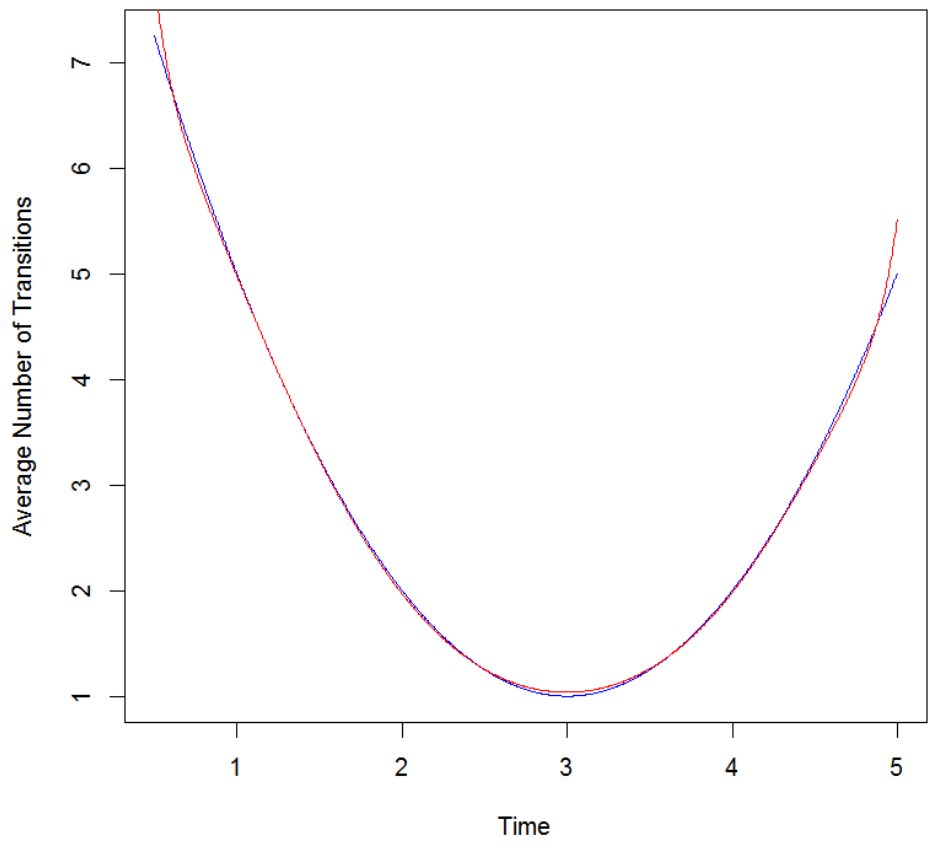

Figure A.55: Quadratic fit for Case 10 with $\lambda=11$ 


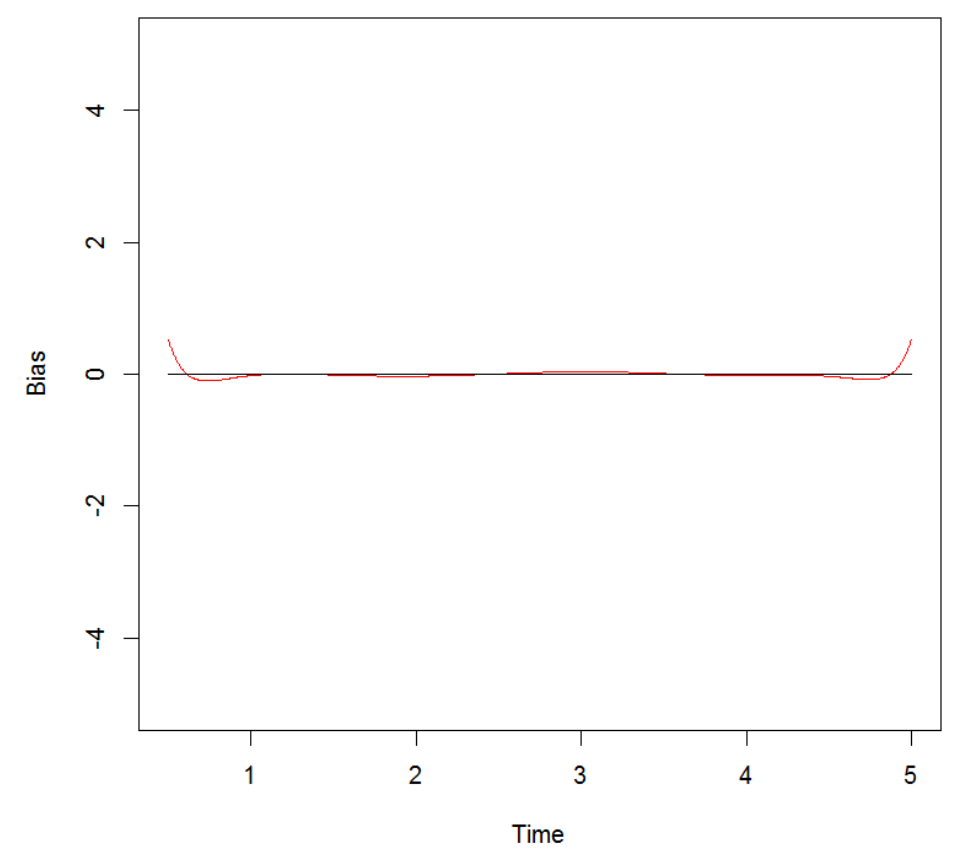

Figure A.56: Bias for the quadratic function of Case 10

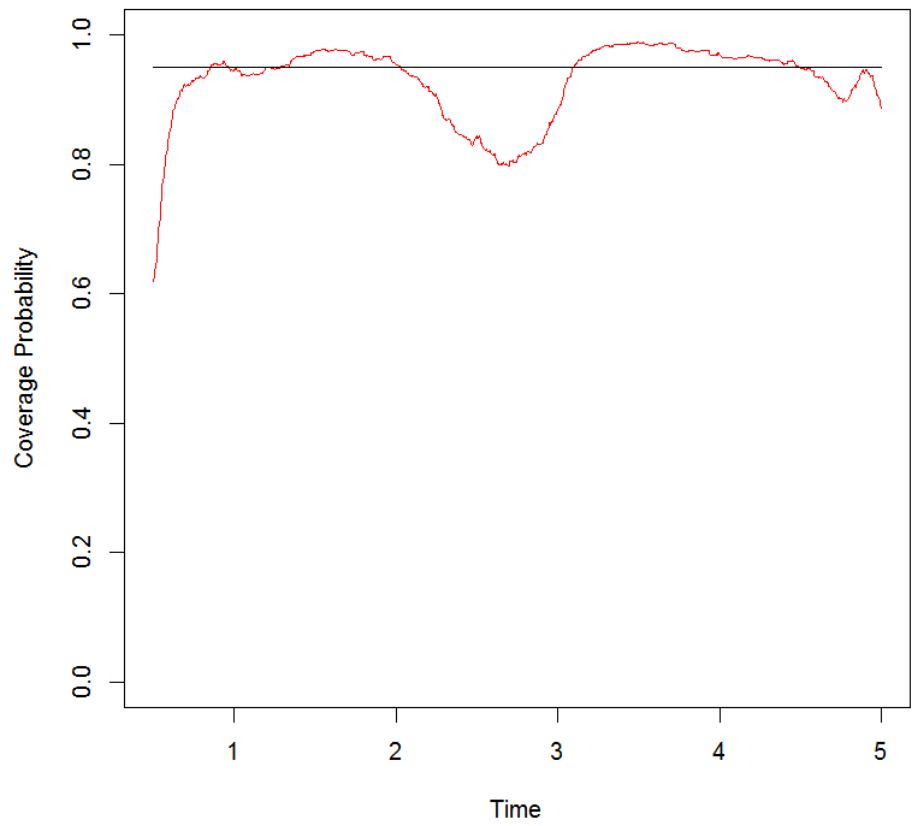

Figure A.57: Coverage probabilities for the quadratic function of Case 10 


\section{A.11 Case 11: Fitting a Log-Normal with the Model Based Penalty}

These are the full set of graphs for the log-normal function case. The log-normal still uses a model based Weibull penalty, but since it is closer to a Weibull than the quadratic, a larger $\lambda$ can be used to fit it best.

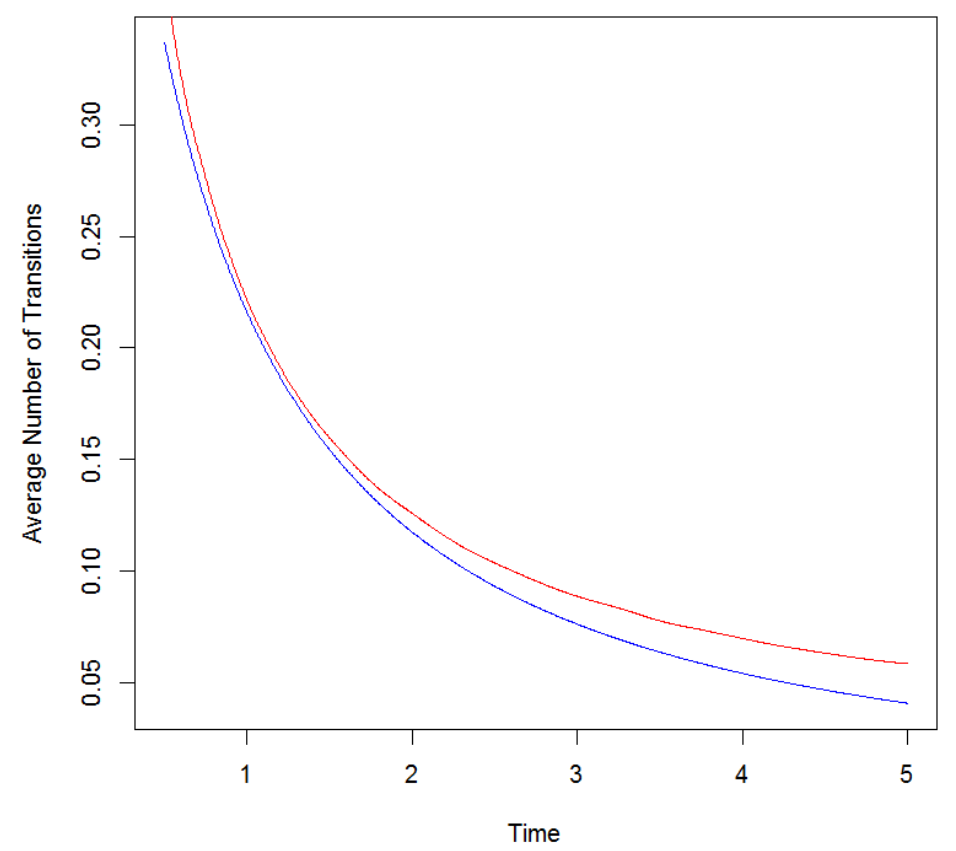

Figure A.58: Log-Normal fit for Case 11 with $\lambda=30$ 


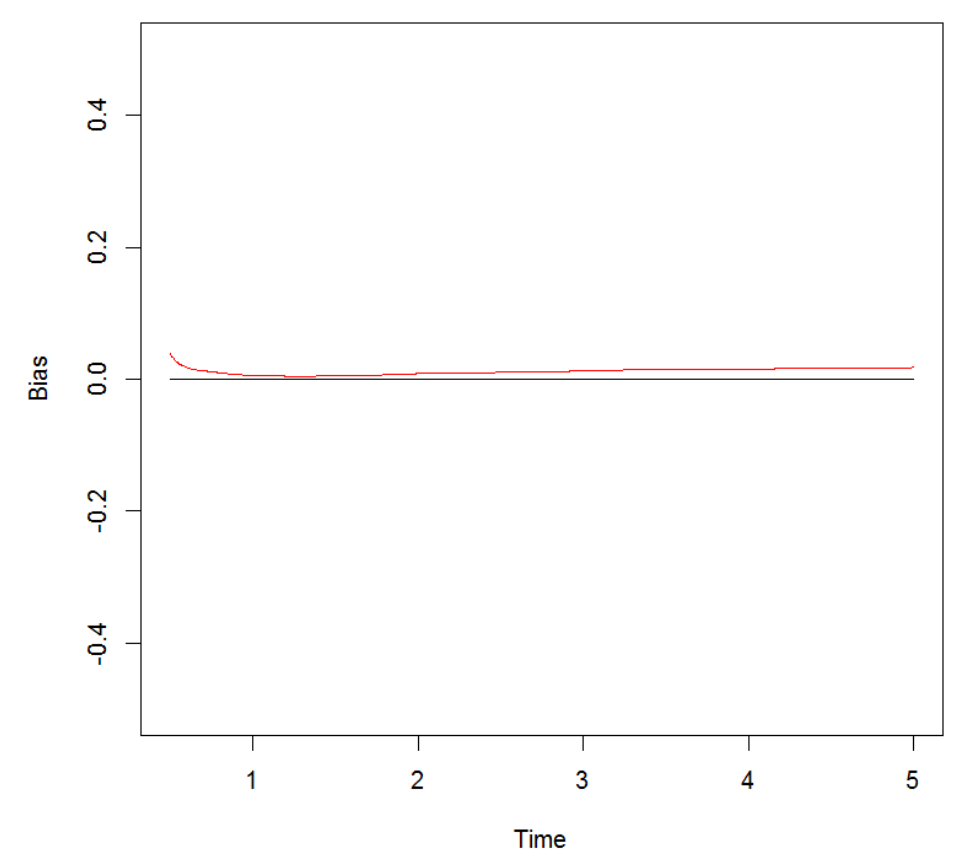

Figure A.59: Bias for the log-normal function of Case 11

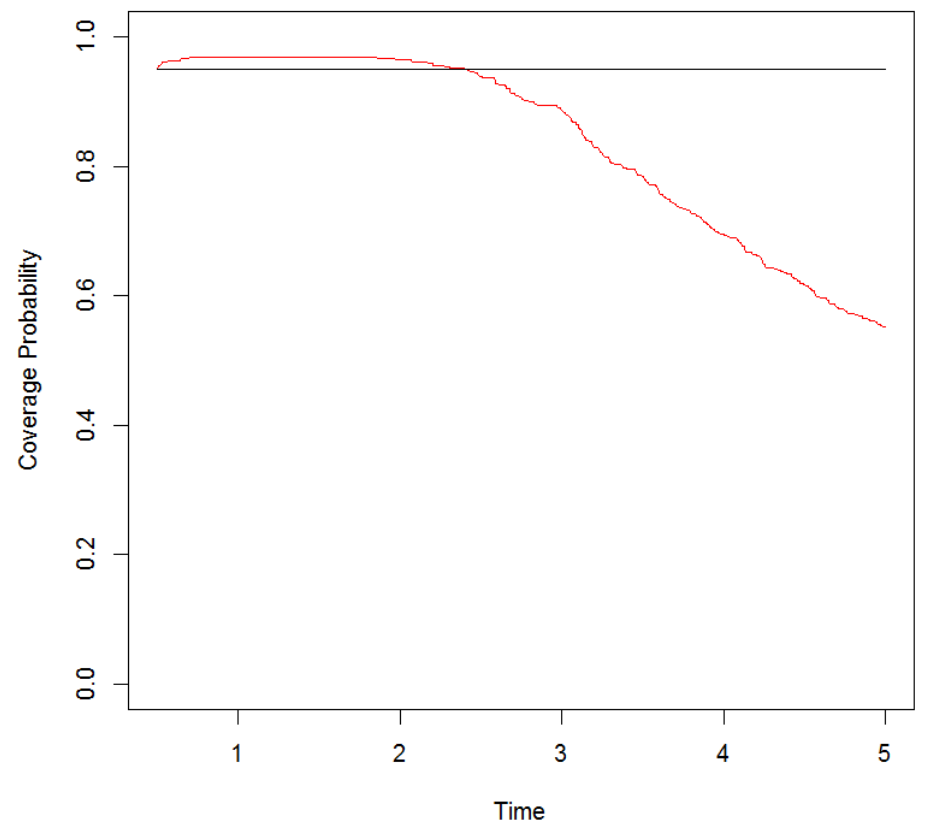

Figure A.60: Coverage probabilities for the log-normal function of Case 11 